\title{
IntechOpen
}

\section{Somatosensory and Motor Research}

Edited by Toshiaki Suzuki

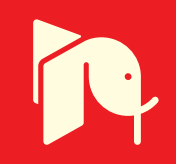





\section{Somatosensory and Motor Research}

Edited by Toshiaki Suzuki 

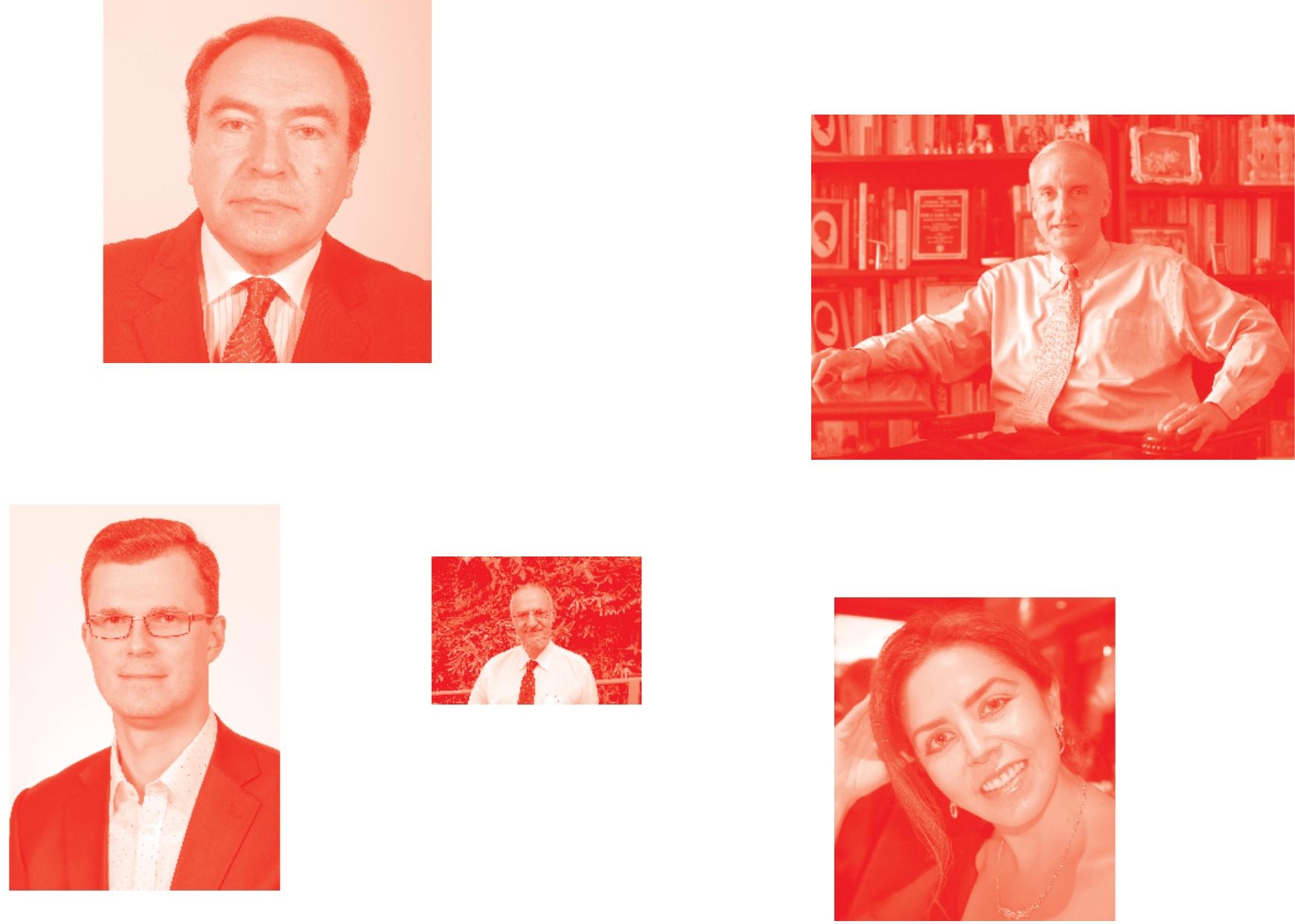

Supporting open minds since 2005
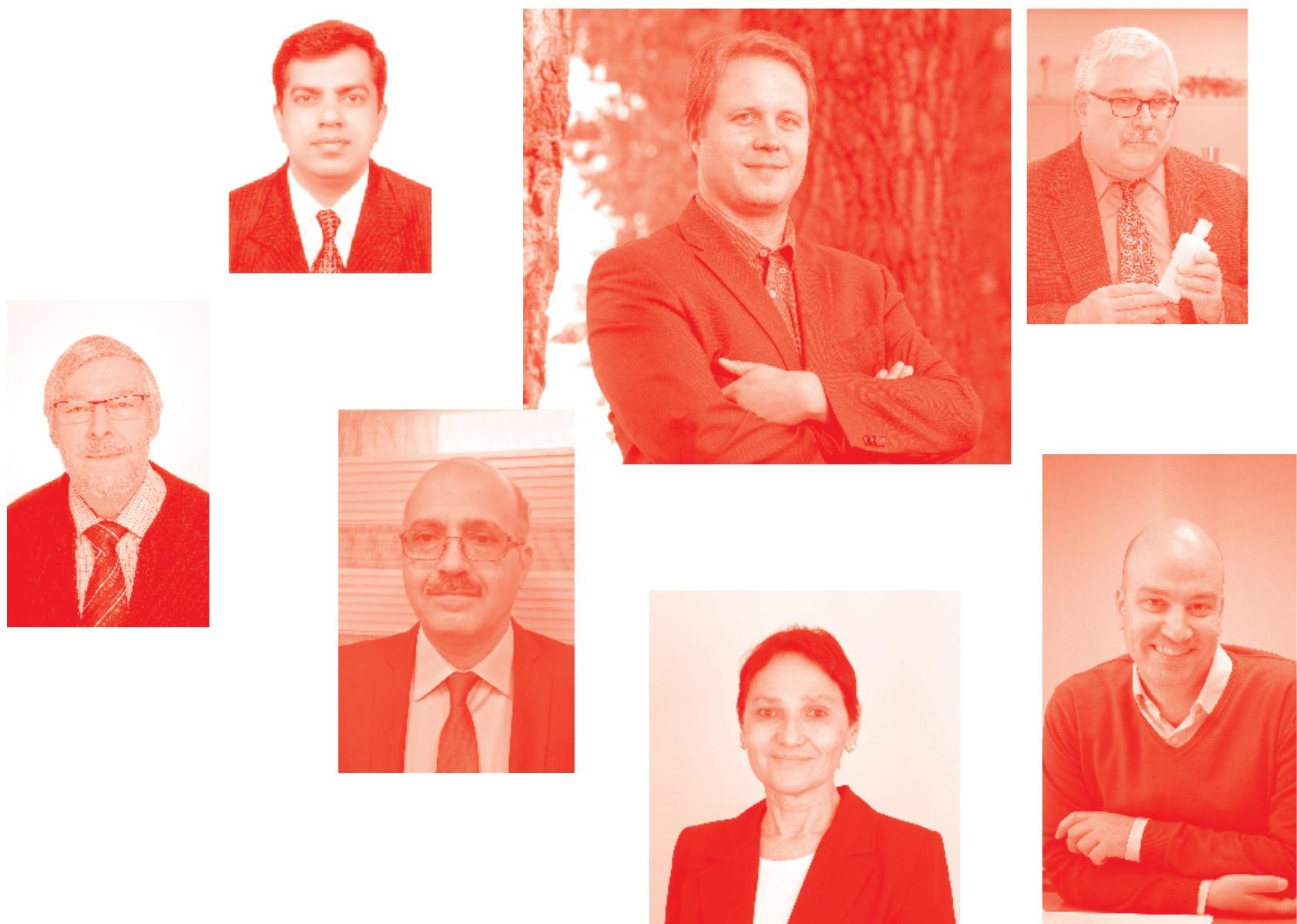
Somatosensory and Motor Research

http : //dx . doi . org/10.5772/intechopen. 80168

Edited by Toshiaki Suzuki

\section{Contributors}

Nina Serhiivna Mishchanchuk, Dmytro Illich Zabolotny, José Antonio A. Vega, Ramón Cobo, Yolanda García-Mesa, Olivia García-Suarez, Lin Yang, Ping Wang, Yoshibumi Bunno, Naoki Kado, Hitoshi Asai, Yuki Fukumoto, Marina Todo, Jorge García-Piqueras, Jorge Feito, José Martín-Cruces

() The Editor(s) and the Author(s) 2020

The rights of the editor(s) and the author(s) have been asserted in accordance with the Copyright, Designs and Patents Act 1988. All rights to the book as a whole are reserved by INTECHOPEN LIMITED . The book as a whole (compilation) cannot be reproduced, distributed or used for commercial or non-commercial purposes without INTECHOPEN LIMITED's written permission. Enquiries concerning the use of the book should be directed to INTECHOPEN LIMITED rights and permissions department (permissions@intechopen.com).

Violations are liable to prosecution under the governing Copyright Law .

\section{(cc) BY}

Individual chapters of this publication are distributed under the terms of the Creative Commons Attribution 3.0 Unported License which permits commercial use, distribution and reproduction of the individual chapters, provided the original author(s) and source publication are appropriately acknowledged. If so indicated, certain images may not be included under the Creative Commons license. In such cases users will need to obtain permission from the license holder to reproduce the material. More details and guidelines concerning content reuse and adaptation can be found at http : //www . intechopen . com/copyright-policy . html.

\section{Notice}

Statements and opinions expressed in the chapters are these of the individual contributors and not necessarily those of the editors or publisher. No responsibility is accepted for the accuracy of information contained in the published chapters. The publisher assumes no responsibility for any damage or injury to persons or property arising out of the use of any materials, instructions, methods or ideas contained in the book.

First published in London, United Kingdom, 2020 by IntechOpen IntechOpen is the global imprint of INTECHOPEN LIMITED, registered in England and Wales, registration number: 11086078 , 7th floor, 10 Lower Thames Street, London, EC3R 6AF, United Kingdom

Printed in Croatia

British Library Cataloguing-in-Publication Data

A catalogue record for this book is available from the British Library

Additional hard and PDF copies can be obtained from orders@intechopen.com

Somatosensory and Motor Research

Edited by Toshiaki Suzuki

p. cm.

Print ISBN 978-1-83880-843-3

Online ISBN 978-1-83962-938-9

eBook (PDF) ISBN 978-1-83962-939-6 


\section{We are IntechOpen, \\ the world's leading publisher of Open Access books}

\section{Built by scientists, for scientists}

\section{$4,900+$ \\ Open access books available \\ $123,000+$ \\ International authors and editors \\ $140 \mathrm{M}+$ \\ Downloads}

151

Countries delivered to

Our authors are among the

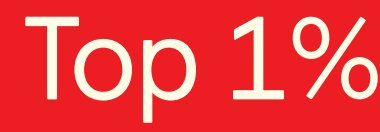

most cited scientists

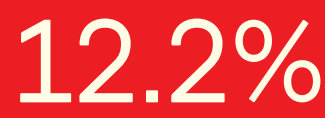

Contributors from top 500 universities

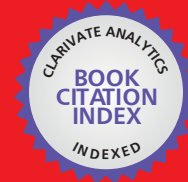

WEB OF SCIENCE ${ }^{\mathrm{M}}$

Selection of our books indexed in the Book Citation Index in Web of Science ${ }^{\mathrm{TM}}$ Core Collection (BKCI)

Interested in publishing with us?

Contact book.department@intechopen.com

Numbers displayed above are based on latest data collected.

For more information visit www.intechopen.com

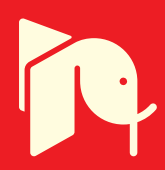





\section{Meet the editor}

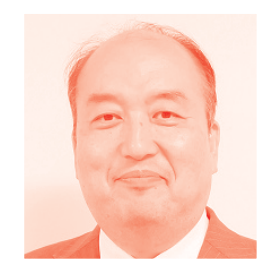

Suzuki Toshiaki, DMSc, is a physical therapist and professor at the Graduate School of Kansai University of Health Sciences, Japan. He investigates the effect and development of neurological physical therapy using electromyography, which he was taught by Fujiwara Tetsuji, MD, and Kimura Jun, MD, emeritus professors at Kyoto University, Japan. He is also interested in the development of Acupoint Stimulation Physical Therapy (ASPT), which combines physical therapy and acupuncture. Dr. Toshiaki has published a number of original papers and books in his field. 



\section{Contents}

Preface

Section 1

Somatosensory Research

Chapter 1

Somatic Nerve Reconstruction and Reinnervation

by Lin Yang and Ping Wang

Chapter 2

The Glial Cell of Human Cutaneous Sensory Corpuscles:

Origin, Characterization, and Putative Roles

by Ramón Cobo, Yolanda García-Mesa, Jorge García-Piqueras,

Jorge Feito, José Martín-Cruces, Olivía García-Suárez and José A. Vega

Chapter 3

Vestibular System: Anatomy, Physiology, and Clinical Evaluation

by Dmytro Illich Zabolotnyi and Nina Serhiivna Mishchanchuk

\section{Section 2}

Motor Research

Chapter 4

Examination of New Parameters from F-Wave Waveform Using

Addition Averaging Method

by Marina Todo

Chapter 5

Motor Imagery for Neurorehabilitation: The F-Wave Study

by Yoshibumi Bunno

Chapter 6

Effective Motor Imagery Application: Examining Spinal Cord

Excitability from the F-Wave and Autonomic Nervous Activity

from LF/HF

by Yuki Fukumoto

Chapter 7

Excitability of Spinal Motor Neurons in the Upper Extremity during Voluntary Movement with Different Difficult Tasks in the Lower Extremity

by Naoki Kado 
Section 3

Relationship between Somatosensory and Motor Research

Chapter 8

119

Functional Role of the Somatosensory Information to Perceive the Standing Position in the Anteroposterior Direction

by Hitoshi Asai 


\section{Preface}

When a person exercises, motor functions such as muscle strength and range of joint motion as well as somatosensory functions that enable the exercise are required. Somatosensory function is very important, especially when performing fine movements.

This book Somatosensory and Motor Research is divided into three sections: "Somatosensory Research," "Motor Research," and "Relationship between Somatosensory and Motor Research.”

In the first section, "Somatosensory Research," chapters provide information on anatomy and physiology as a basis for explaining how to treat sensory disorders. In the second section, "Motor Research," chapters examine the relationship between the evaluation of F-wave, an index of excitability of spinal cord horn cells involved in motor function, and motor function. In the final section, "Relationship between Somatosensory and Motor Research," chapters address the effects of somatic sensation on standing function.

We would like to thank all the authors who contributed to this volume. They are all great researchers, and many are involved in rehabilitation. As such, I am convinced that this book will revolutionize the field of physical rehabilitation.

We would also like to thank Ms. Rebekah Pribetic, Author Service Manager at IntechOpen, for her help in the publication of this book.

Suzuki Toshiaki, PT, DMSc Professor,

Graduate School of Kansai University of Health Sciences, Osaka, Japan 

Section 1

\section{Somatosensory Research}





\title{
Somatic Nerve Reconstruction and Reinnervation
}

\author{
Lin Yang and Ping Wang
}

\begin{abstract}
Somatic nerves include somatic sensory and somatic motor, both of which are part of the peripheral nervous system. When somatic motor nerve or somatosensory nerve produces injury, then it belongs to peripheral nerve injury. Peripheral nerve injuries are common disease and complex process in clinical surgery. The severe physical dysfunctions such as motor/sensory loss, dyskinesia, and nutritional disorders in the area innervated caused by the peripheral nerve injury and even leave a lifelong disability. Therefore, somatic nerve reconstruction is essential and remains a major challenge for surgeons. Therapeutic methods include neurorrhaphy and nerve transfer or redistribution reconstruction; traditional Chinese medicineassisted nerve regeneration and repair methods have been increasingly improved, even the functional recovery of peripheral nerve has not made breakthrough progress. All kinds of reconstruction and neural rehabilitation technologies have their own advantages, but they all have some limitations. Although great progress has been made in nerve electrophysiology and microsurgery, the results are still unsatisfactory. This chapter reviews the application of peripheral nerve reconstruction technology and briefly describes the advantages and disadvantages of each method, which will be useful for the selection of clinical treatment.
\end{abstract}

Keywords: somatic nerve, peripheral nerve injury, neural rehabilitation, reinnervation, traditional Chinese medicine

\section{Introduction}

The peripheral nerves are all nerves, except the brain and the spinal cord, and peripheral nerves are composed of nerve fibers. The longer protrusions of the neurons are surrounded by an insulating sheath of myelin and nerve membranes to form nerve fibers. Neurons are classified into motor neurons and sensory neurons according to their function and the direction of nerve conduction. Motor neurons conduct signals from the spinal cord and brain to the effector, and sensory neurons transmit excitability to the central nervous system. The motor and/or sensory nerve fiber bundles form the corresponding motor, sensory, or mixed nerves. Therefore, matching the corresponding bundle branches during nerve repair is of great significance for nerve regeneration. This article discussed the common clinical methods of nerve anastomosis and nerve transplantation and analyzes the characteristics of various neurosurgical reconstruction methods and the effects on somatosensory function recovery. At the same time, this article will list several recognized and effective methods of traditional Chinese medicine neuro rehabilitation, aiming to provide options for somatosensory rehabilitation after peripheral nerve injury. 


\section{Nerve transplantation}

The concept of nerve transplantation is to repair peripheral nerve injury caused by various injury factors by means of nerve autograft and/or nerve allograft. It is a major alternative technique for nerve neurorrhaphy, applied to the clinical process of nerve neurorrhaphy due to excessive stretching of the nerve leading to partial or permanent ischemia. At present, peripheral nerve transplantation has developed some major types of transplantation such as nerve autograft and nerve allograft. The common transplantation methods used in surgical operations are nerve trunk transplantation, nerve cable transplantation, and nerve bundle transplantation.

\subsection{Neurorrhaphy}

\subsubsection{End-to-end (ETE) neurorrhaphy}

The peripheral nerve injuries lead to different types and degrees of injuries; for the treatment of peripheral nerves disorders, microsurgery plays a very important role. It is the neurorrhaphy of the broken port to restore the anatomical continuity of the nerve and enable the distal nerve fibers to grow into the corresponding target tissue, so as to restore its sensory and motor functions to the greatest extent. At present, the method commonly used in clinical practice to repair nerves with a defect of a short segment of nerve is direct neurorrhaphy (Figure 1A). ETE neurorrhaphy [1] is the current gold standard for clinical repair of nerve injury, with the development of modern microsurgical technology, which has developed from epineurium suture to small gap bridging method and sticky method under the microscope, the nerve technology of conventional silk suture repair has reached a higher level. ETE neurorrhaphy is available, reduces the degree of damage to the nerve itself and surrounding tissues, reduces inflammation and scar formation, and
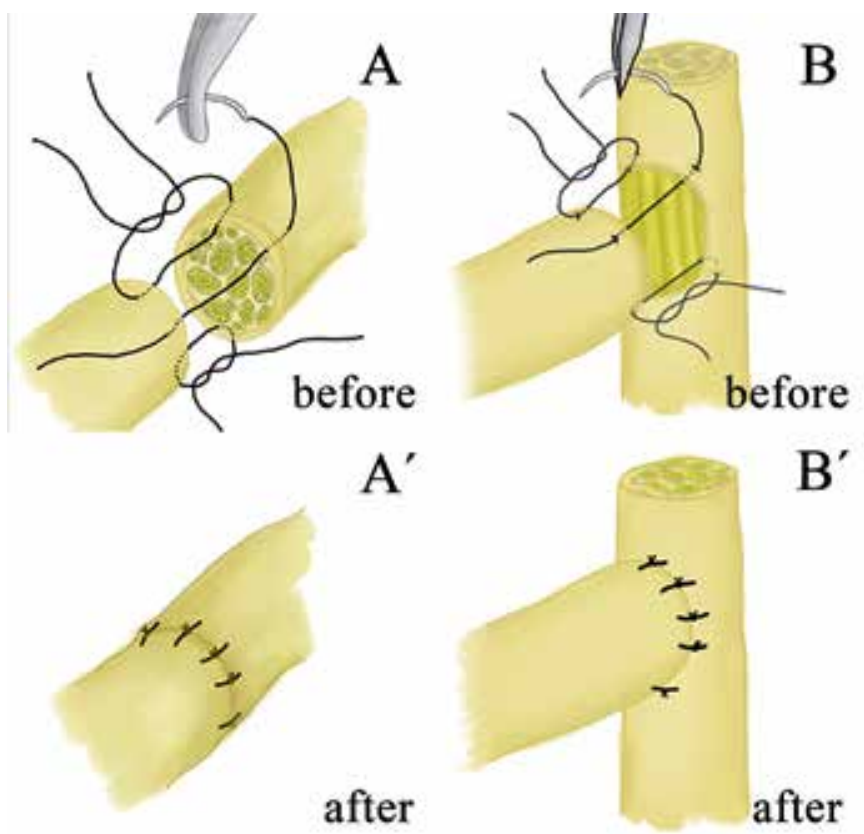
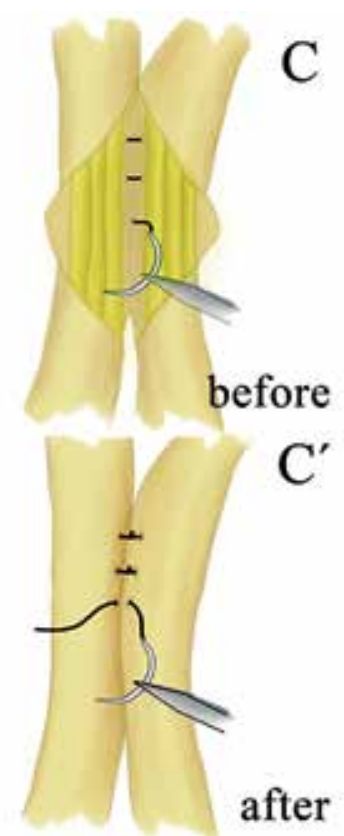

Figure 1.

The schematic diagram of neurorrhaphy (drawn by Lin). 
is conducive to nerve regeneration and maturation. However, ETE neurorrhaphy cannot repair long nerve defects, which is larger than $2 \mathrm{~cm}$.

\subsubsection{End-to-side (ETS) neurorrhaphy}

ETS neurorrhaphy [2] is to sew (Figure 1B) the distal end of the injured nerve to the side adjacent to the normal nerve and to regenerate the nerve through sudden shoots of the collateral axis, thereby restoring the sensory and motor functions of the injured innervation area. This surgical method is simple and requires only anastomosis without tension. The donor nerve can be opened with or without the window of the epineurium. After ETS neurorrhaphy, the function of the donor nerve is not affected. The biggest advantage of this method in repairing nerve damage is that it does not need to consider the distance of the damaged nerve proximal defect, and it does not affect the function of the donor nerve. In 1994, Viterbo et al. [3] first proposed a rat model, which sutured the distal stump of the rat peroneal nerve to the side wall of a healthy tibial nerve on the same side. Experimental studies have found that after ETS neurorrhaphy, injured nerves regenerate, and targeted organ function can be restored. Tarasidis et al. [4] confirmed that sensory nerves can also achieve nerve regeneration through ETS neurorrhaphy. The effect of nerve regeneration depends on many factors, such as contact area and anastomosis angle. Jia et al. increased the contact area of the nerve stump and repaired the nerve with ETS neurorrhaphy, and the nerve fibers regenerated well, increasing the contact area of the nerve stump could obtain more effective nerve regeneration. Yu et al. [5] studied whether sensory nerve induces motor nerve regeneration and suggested that ETS neurorrhaphy of a sensory nerve gives rise to successful motor nerve reinnervation.

Regenerating fibers are produced by normal nerve trunks through collateral sprouting after nerve ETS neurorrhaphy. The level of regenerative nerve function recovery is similar to the effect of ETS neurorrhaphy and has [6] the same effect as nerve transplantation. Defective nerves can achieve self-connection of regenerating fibers by bilateral anastomosis with normal nerves, but this connection plays only a minor role in the recovery of injured nerve function, and the collateral regeneration fibers from the normal neural stem play a major role. At the same time, anastomosis may become a new method for the prevention and treatment of stump neuroma.

Experimental research and preliminary clinical practice [7] prove that nerve ETS neurorrhaphy is a promising method for treating peripheral nerve injury. Especially, it is a suitable choice when repairing a large segment of nerve defect, and ETE neurorrhaphy cannot be applied, but there are few studies in this area, and whether the ETS neurorrhaphy is superior and needs further research.

\subsubsection{Side-to-side (STS) neurorrhaphy}

As the distal end of the injured nerve and the adjacent healthy nerves are opened and sutured on the opposite side (Figure 1C), two nerve channels are established. Transportation reaches the damaged nerve and reaches the target organ to provide nutrition or grow lateral buds, thereby [8] promoting regeneration and repair of the damaged nerve, so as to restore part of the nerve's function. It is obvious that nerve regeneration after ETS and STS neurorrhaphy is not enough to replace the function of the original inner nerve in dominate muscle function.

Peripheral nerve STS neurorrhaphy is one of the effective methods for treating spastic cerebral palsy of the limbs. The spastic muscle group can obtain some normal innervation after operation to change the localization of the cerebral cortex. 
Zhou et al. [9] established a model by ligating and fixing the proximal peroneal nerve transection of the right common peroneal nerve in New Zealand rabbits and opening a window of the outer membrane of distal and ipsilateral tibial nerves and anastomosing them with lateral suture, concluding that this method can effectively protect the motor end plate function of the denervated muscles. STS neurorrhaphy [10] is a remedial treatment for damaged nerves that cannot be repaired by conventional methods, such as high injury or incomplete injury such as brachial plexus lower trunk or sciatic nerve injury.

\subsection{Nerve autograft}

Nerve autograft is a method of suturing and repairing the injured area of the autologous nerve with free autologous nerve. Philipeaux et al. first used the free nerve transplantation to repair hypoglossal nerve defect in 1870, which became the initiation of autologous transplantation. Oberlin et al. [11] reported for the first time that the ipsilateral 1/10 ulnar nerve bundle branch was transposed and anastomosed to the musculocutaneous nerve and biceps brachii muscle branch to repair brachial plexus nerve injury. Since then, the operation has been widely used and continuously improved. Contralateral C7 nerve transposition was first created by Gu et al. [12] for the treatment of brachial plexus root avulsion in 1986, which developed a contralateral C7 vertebral body anterior displacement (in combination with nerve graft) to repair a total brachial plexus avulsion. Nerve autograft throughout hundreds of years' researching has developed into pedicled autologous nerve transfer and free autologous nerve transfer. Pedicled autologous nerve transplant consists of pedicled autologous nerve transfer and pedicled vascular autologous nerve transfer. Free autologous nerve transfer includes traditional autologous nerve transfer, such as cable, trunk, and vascular nerve transfer [13]. Nerve autograft have incomparable advantages over other materials, such as nonantigenicity; orderly arrangement of axons regenerated in vivo; accurate access to target tissues, organs, and blood supply; and low rejection by the body. Autograft have remained as the standard for the nerve grafting material. Therefore, nerve autograft is the golden standard treatment for the majority of clinical diseases in the current peripheral surgery for repairing peripheral nerve defects [14].

However, the limited sources are liable to cause nerve dysfunction. Sensory deficiency and scar formation in donor site, which result in nerve regeneration, were still being pended. Besides, insufficient blood supply and connective tissue hyperplasia have also interfere with its development [15]. Therefore, studies on improving autologous peripheral nerve blood flow, especially microcirculation and blood-nerve barrier restoration, are intended to provide blood flow guarantee for the survival of nerve transplantation segments and are a prerequisite for improving nerve regeneration [16]. Furthermore, increasing Schwann cells and forming Schwann cells with the same number of nerve fibers make nerve fibers to regenerate faster than connective tissue hyperplasia and can achieve better neural regeneration effect [17].

\subsection{Nerve allograft}

In order to overcome the main shortcomings of nerve allograft in repairing peripheral nerve injury, related scholars have explored a new repairing methodnerve allograft. In 1885, Albert made the first attempt of human nerve allograft. In recent hundred years, it was found that nerve allograft have many advantages, such as no secondary damage on patients, availability of various types of nerve segments, and sufficient sources. It is regarded as one of the new development directions of 
nerve repair and an alternative technical of nerve autograft. But after fresh nerve allograft transplantation by Davis et al. [18], it was found that the area where it was operated on transplantation could not be fully restored. Considering that the transplanted nerves ended with central necrosis and fibrous scarring, Sunderland believed that using fresh nerve allograft was a failure [19]. At present, it is generally accepted in academia that rejection reaction between graft and recipient is the main factor affecting functional repair. Therefore, the key to the success of transplantation is whether immune rejection can be suppressed or not [20]. At present, the main research direction is to explore how to remove the antigenicity of nerve allograft. Radiation-irradiation, cryopreservation, and high temperature preservation are the methods to reduce immunogenicity, but the effect of these pretreated nerve allograft on promoting nerve regeneration is significantly weaker than that of fresh autologous nerve transfer [21]. On these above shortcomings, many domestic and foreign scholars have carried out various animal experiments, but no breakthrough has been made.

\subsection{Targeted reinnervation (TR)}

In recent years, scholars have shown great interest in how to enhance the prosthetic somatosensory and motor function of patients with traumatic neurological injuries wearing prostheses [22-25]. Northwestern University and the Chicago Institute of Rehabilitation for the first time proposed the use of TR technology to further improve the flexibility and accuracy of prosthetic sensation and motor function and successfully applied the technology to a male patient with a transshoulder fracture with myoelectric prosthesis [26]. Conventional myoelectric prosthetics rely on surface electromyographic amplitude changes for simple displacement of the hands and elbows [27]. Although such artificial limbs breakthrough the bottleneck of the robotic arm being difficult to move autonomously with personal consciousness, however, once the degree of amputation reaches above the elbow joint, the acquisition of electromyography (EMG) signals is extremely hard [28]. Due to insufficient accuracy and sensitivity, it is difficult to process the complex signals on the EMG of the wrist, thumb, and other parts [29]. TR transplants the amputated patient's nerves to the target muscles to avoid atrophic atrophy of the performing muscles and loss of motor function. By separating the original nerves that dominate the target muscles, it reestablishes the dominant relationship between the remaining important nerves and the target muscles [30]. Muscles are bioamplifiers for stump motion signals, which can convert cortical signals transmitted by newly implanted nerves into myoelectric signals to control prosthetic activity.

TR can reestablish the nerve-muscle innervation relationship. It can more intuitively use myoelectric signals to manipulate limb movements and improve myoelectric prosthetic control. The key to control is that each reinnervated muscle during TR surgery must only generate an EMG signal in response to transplantation to that nerve [31]. In 2009, Kuiken et al. [32] performed TR surgery on a woman with a shoulder joint amputation caused by a car accident. The patient's residual ulnar nerve was connected to the pectoralis major muscle, anastomosis of the median nerve with the pectoralis major at the sternum, and the musculocutaneous nerve was connected to the clavicle head of the pectoralis major muscle. At the same time, the primordial nerves that dominate the above muscles were separated, and the distal end of the long thoracic nerve was anastomosed with the radial nerve end, the distal end of the intercostal arm nerve was anastomosed with the median nerve side, anastomosis of the distal end of clavicle epithelial nerve with the ulnar nerve (see Figure 2). Six months after TR, the electrodes widely placed 
on the patient's chest could clearly record all myoelectric signals connected to the nerve. After rehabilitation training, the patient was very skilled in the operation of the prosthetic hands and elbows. When opening, closing, bending, or correcting the elbow, the prosthesis can respond in a timely and accurate manner. Later, after transforming the traditional three-degree-of-freedom myoelectric prosthesis [33] into six free vacation limbs (shoulder flexion, humerus rotation, elbow flexion, wrist rotation, wrist flexion, and hand control), including nonpowered locking shoulder, passive humeral rotator, powered elbow, motorized wrist, and motorized

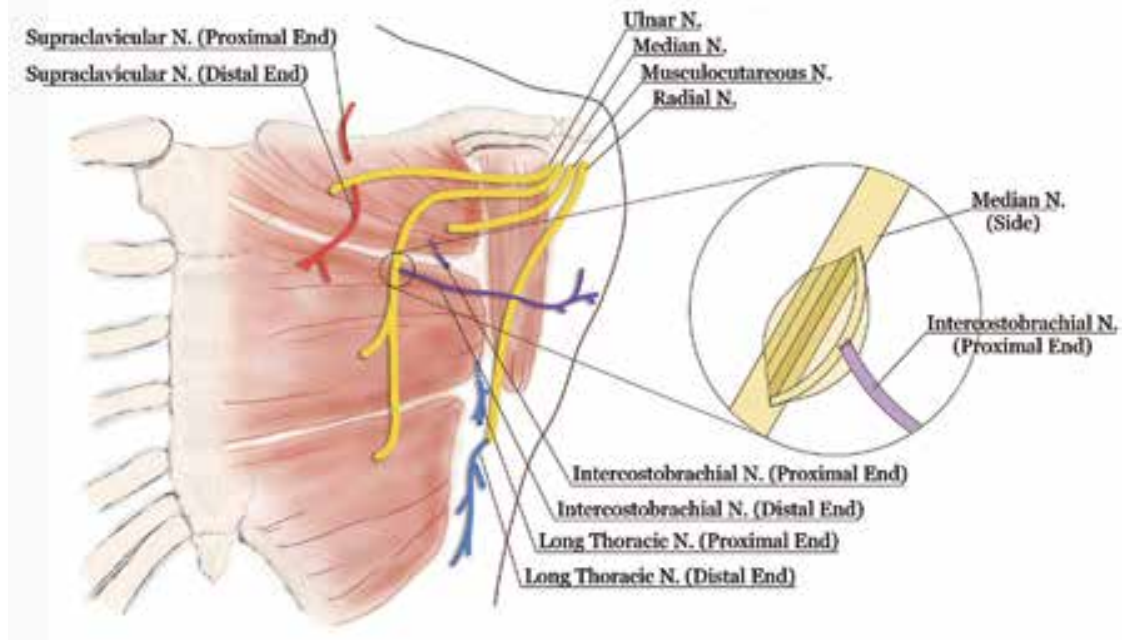

Figure 2.

Schematics of TR surgery: the patient's residual ulnar nerve was connected to the pectoralis major muscle, anastomosis of the median nerve with the pectoralis major at the sternum, and the musculocutaneous nerve was connected to the clavicle head of the pectoralis major muscle, the primordial nerves that dominate the above muscles were separated, and the distal end of the long thoracic nerve was anastomosed with the radial nerve end, the distal end of the intercostal arm nerve was anastomosed with the median nerve side, anastomosis of the distal end of clavicle epithelial nerve with the ulnar nerve (drawn by Lin).

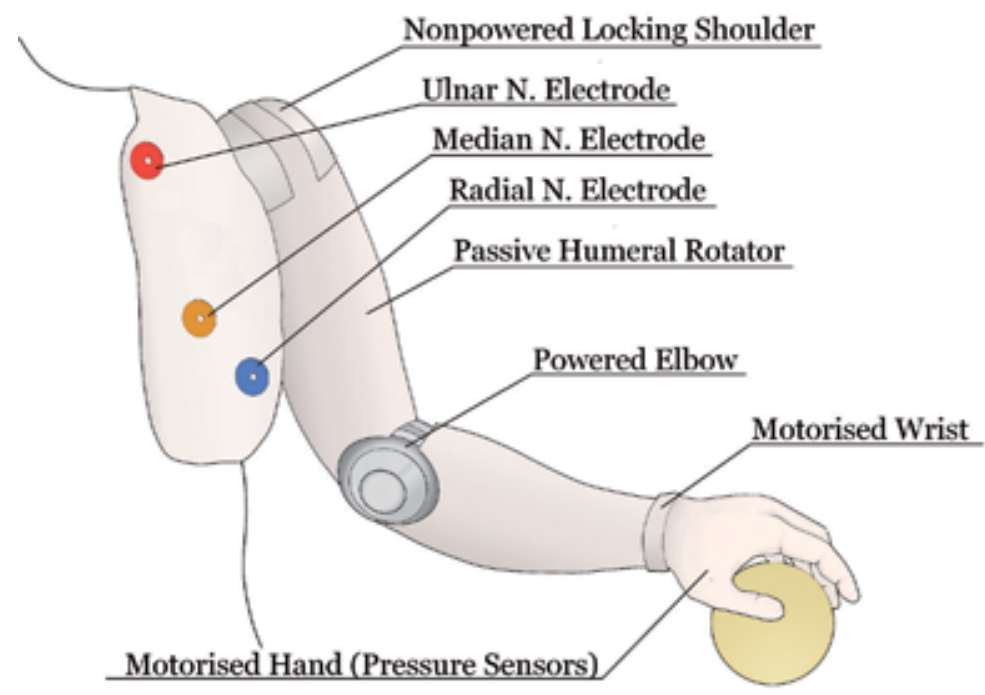

Figure 3.

Six free vacation limbs including nonpowered locking shoulder, passive humeral rotator, powered elbow, motorized wrist, and motorized hand with pressure sensors can lead motor of shoulder flexion, humerus rotation, elbow flexion, wrist rotation, wrist flexion, and hand control (drawn by Lin). 
hand with pressure sensors (see Figure 3 ). The patient could perform various movements with high flexibility. After being equipped with pressure sensors at the hand control position, the thumb, index finger, and middle finger could cooperate to complete the basic grasping action.

Amputees wearing prosthetics need not only to have a flexible motor function but also to have a precise sensory function. Somatosensory signal input is an important part of the upper limbs performing motor functions, especially the fingertips are largely dependent on tactile perception of the surrounding environment information [34]. The field of perceptual tactile sensation is very complicated. In 2009 (Sensinger et al.) [35], skin touch tests were performed on three patients undergoing TR surgery. The first subject (S1) undergoes directional transplantation of the musculocutaneous nerve, median nerve, radial nerve, and ulnar nerve to the head of the pectoralis major clavicle and the head of the pectoralis major sternum.

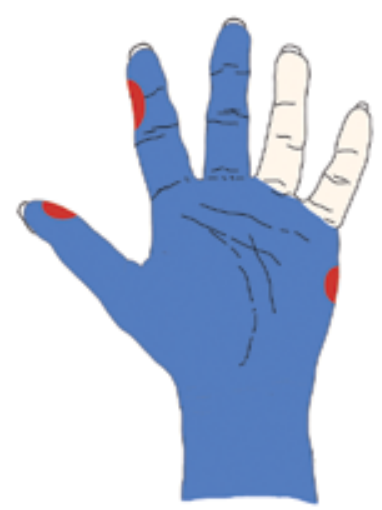

Palmar Hand

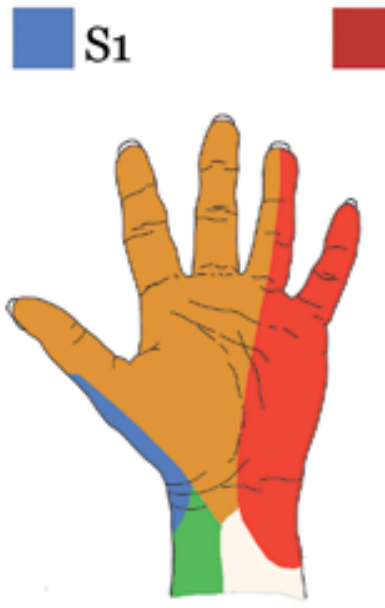

Palmar Hand

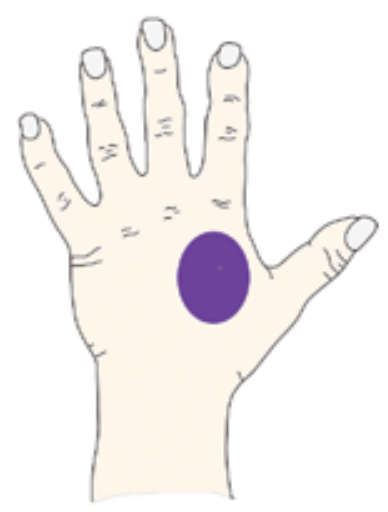

Dorsal Hand
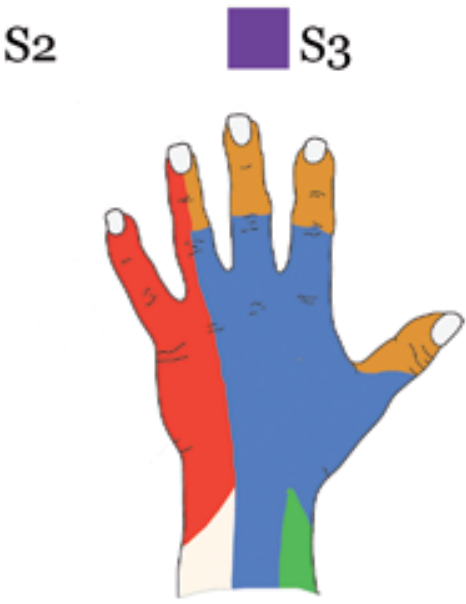

Dorsal Hand

Median N.

Ulnar N.

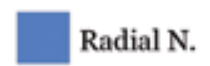

Musculocutareous N.

Figure 4.

Diagrams of skin sensation after TR surgery (referred to Sensinger et al): S1 skin test indicated that the tactile position was located on the back of the hand; S2 skin test showed that the tactile position was located on the outer edge of the index finger, thumb, and palm outer edge; and S3 skin test showed that the tactile position was located on the three fingers on palm and palm side. 
Five and a half years of the innervation skin test indicated that the tactile position was located on the back of the hand. For the second subject (S2), the ulnar and musculocutaneous nerves were redirected to the medial and lateral halves of the clavicular head of the pectoralis major. A two-and-a-half year innervation skin test showed the tactile position on the outer edge of the index finger, thumb, and palm outer edge. The third subjects (S3) who underwent TR surgery of the distal radial and median nerves to the lateral head of the triceps brachii and the medial head of the biceps brachii. After 1 year of innervation skin test, the tactile position was located on the three fingers on palm and palm side (see Figure 4). Although compared with the normal sensory ability of innervating the hand, TR surgery failed to make the patient's prosthesis completely respond to sensory feedback, but the main sensory nerve pathways were basically established [36], especially the median nerve function recovery was ideal [37].

Nerves are prone to cause symptomatic neuromas during the repair process after traumatic injury. Clinically, neuroma resection or nerve traction is often used to relieve the pain symptoms of neuromas, but nearly $35 \%$ of patients do not control their pain symptoms after treatment. TR surgery connects the residual nerve with the target muscle or the corresponding nerve [35, 38]. Although it is difficult to ensure a one-to-one correspondence, compared with autologous nerve transplantation or transplantation, the cost is much smaller. Northwest Hospital counted 100 patients who underwent TR surgery and found that only one patient had symptomatic neuroma after surgery [39]. The data confirmed that TR had certain clinical significance for the prevention and treatment of neuromas.

In addition, due to the limited application of the lower limb electromyography device, the clinical effect of TMR in lower limb amputees is still unclear. At the same time, lower limb metabolic energy costs are higher. There are also complex problems such as sitting posture, asymmetric gait, and slope walking, which needed to carry out in-depth research to evaluate the application value of TMR.

\section{Traditional Chinese medicine treatment}

There is no such thing as "peripheral nerve injury" in traditional Chinese medicine, but the symptoms after peripheral nerve injury are similar to "tendon injury" and "paralysis syndrome" in the category of traditional Chinese medicine meridian. Chinese medicine believes that the pathogenesis of this type of disease is Qi and blood disorders. Local stasis after peripheral nerve injury can cause Qi stagnation, Qi and blood circulation disorders in the meridians, and insufficient nutrition at the injury site, resulting in local tissue ischemia and abnormal body function. If the trauma severely causes the body to lose blood and Qi instantly, the Qi and blood cannot be delivered to the body, the limbs will become cold, and Qi stagnates blood stasis and then muscle atrophy, resulting in peripheral nerve somatosensory dysfunction [40]. Therefore, in view of the mechanism of Qi and blood dysfunction, Chinese medicine believes that it should be based on imagination and deficiency and based on Qi deficiency and blood stasis as the standard. Rehabilitation treatment methods including acupuncture, massage, fumigation, and heat compress can achieve certain results.

Acupuncture uses acupuncture to penetrate acupuncture points in the human body to achieve the purpose of treatment. For example, acupuncture at Jiquan can help regulate the balance of Qi and blood in the human body and enhance the smoothness of Qi and blood in the body; acupuncture at the Yangming Meridian and Sun Meridian can unblock meridians and reconcile Qi and blood [41]. Modern medical research shows that acupuncture can reduce myelin shedding, prevent axon 
disintegration, and protect damaged neurons. He et al. [42] used acupuncture at Baihui (GV20), Fengfu (GV16), Dazhui (GV14), and Shenzhu (GV12) for patients with upper limb nerve injury and acupuncture at Zizhong (GV6), Mingmen (GV4), Yaoyangguan (GV3), and Yaoshu (GV2) for patients with lower limb nerve injury and found that the speed and range of motor nerve transmission were large after treatment, which suggested that acupuncture at various points could effectively enhance nerve excitability and relieve conduction dysfunction; in addition, electroacupuncture could accelerate blood flow in blood vessels, improve blood supply, and thus improve the degree of muscle atrophy. Li et al. [43] found that the content of myelin basic protein MBP in the nerve tissue of the sciatic nerve injury rat model group after acupuncture was significantly higher than that of the control group. The increase of MBP and its antibody expression can promote the release of neurotrophic factor 3 , reduce the shedding of myelin sheath, inhibit the apoptosis of nerve cells, and promote the repair of injured sciatic nerve.

Moxibustion ignites the moxa with fire, burns the acupuncture points, penetrates the heat into the skin, and warms the blood. Moxibustion stimulates acupoints on the body surface in this way and regulates the functions of Qi, blood, and internal organs through the conduction of meridians throughout the body. After peripheral nerve injury, the use of moxibustion can significantly reduce the degree of muscle atrophy and help restore muscle regeneration [44]. The early use of moxibustion can alleviate muscle atrophy and restore neuronal function.

The massage technique can relax the muscles, clear the vital energy, loosen the joints, and relieve the pressure, thereby alleviating the local muscle spasm and promoting the blood circulation. In traditional Chinese medicine treatment of peripheral nerve injury, massage can improve the behavior and morphology of peripheral nerve injury. Lu et al. [45] found that the myelin sheath structure of myelinated nerve fibers in rats with sciatic nerve injury treated by massage tends to be complete. The morphology and structure are arranged neatly, and the thickness of myelin sheath and axonal diameter are significantly restored, indicating that massage can effectively promote the recovery of somatosensory function after sciatic nerve injury.

Traditional Chinese medicine fumigation is a treatment method that acts on the body through the permeability of the skin by virtue of the dual effects of medicine and heat. Traditional Chinese medicine fumigation therapy is based on the principle of syndrome differentiation and treatment of traditional Chinese medicine, and according to the needs of disease treatment, certain Chinese medicines are selected to form fumigation prescriptions. The combination of medicinal power and thermal power plays a role. During fumigation, due to the warm and hot stimulation, the medicine gas and heat can reach the disease site directly, which can promote the blood circulation and lymph circulation of the local and the whole body; improve the nutrition of the local tissues; promote the whole body function; dredge the meridians and collaterals; promote the circulation of Qi and blood; eliminate swelling and stasis; relieve the tension and pressure of the local tissues; relieve the tension or rigidity of the skin, muscles, tendons, and ligaments; and make the joints and limbs move flexible to promote early recovery of limb function [46].

After acupuncture, moxibustion, and fumigation act on acupoints and meridians on the body surface, the stem or peripheral nerves are stimulated, and the paralyzed nerves are passively excited and reflexive, which promote nerve fiber repair. Therefore, the clinical development of modern rehabilitation methods combined with traditional Chinese medicine acupuncture and massage is an effective method to relieve pain, improve peripheral nerve somatosensory function, and prevent muscle atrophy after brachial plexus injury. It is hoped that it will be promoted in clinical applications. 


\section{Summary}

Nerve injury is a common clinical disease with an incidence of about 1 million cases per year [47]. According to foreign data, the excellent and good rate of repairing peripheral nerve injury is less than half [48], and the disability rate is high. At present, primary doctors are familiar with peripheral nerve injury, but their understanding and practical operation of the theory of peripheral nerve injury still have many shortcomings and even cannot suture the injured nerve with high quality. The situation is very serious. Gu [49] proposed that the key points of successful regeneration of peripheral nerves include the following:

- survival of injured neuron cell bodies;

- budding and extension of proximal axons and growth into distal endometrial basement membrane canals of the same function;

- reconstructing synaptic connections between regenerated axons and corresponding target organs, restoration of target organs of nerve reinnervation;

- synthesis of neurotransmitters and related enzymes of neurons. Specific substances restore nerve conduction, axonal transport, and control of target organs;

- the central nervous system can collect and integrate peripheral nerve signals.

At present, surgeons have limited ability to intervene in the repair and regeneration of peripheral nerve injuries clinically. For instance, they can only intervene including the choice of repair time, repair methods, and rehabilitation retraining. Therefore, they should grasp the "golden time" of 1-3 months after injury, adhere to the concept and technology of microrepair, and pay attention to major basic principles such as normal tissues, tension free, the matching of nerve bundle type and function, base bed, and blood supply.

In summary, with the rapid development of modern medical technology, the continuous research of tissue genetic engineering, and the development of traditional Chinese medicine, the methods and types of peripheral nerve repair after injury are much more in depth. The treatment techniques described in this chapter enrich the treatment methods and therapeutic effects and should be selected according to the specific conditions in clinical practice. However, there are still many problems, such as the promotion of many drugs on nerve regeneration and repair, but the specific mechanism is still unclear, which needs further study [50]. Therefore, great efforts still need to be made to find new repair methods in order to improve the effect of nerve recovery.

\section{Acknowledgements}

The completion of the chapter is attributed to many people's support and encouragement. First and foremost, I want to extend my heartfelt gratitude to supervisor, Professor Guanglin Li, whose patient guidance, valuable suggestions, and constant encouragement make me successfully complete this chapter. He gives me much help and advice during the whole process of my writing, which has made my accomplishments possible. 
Also, I would like to express my sincere gratitude to the fund supports of National Natural Science Fundation of China (Grant Nos. U1613222, 81960419, and 81760416) and also to thank the Key Laboratory of Human-Machine-Intelligence Synergic System, Shenzhen Institutes of Advanced Technology, Chinese Academy of Sciences.

Last my thanks would go to my student, Ms. Qianling Zhang who search and collect of references for the completion of this chapter.

\section{Author details}

Lin Yang ${ }^{1}$ and Ping Wang ${ }^{2 *}$

1 Department of Human Anatomy, Zhuhai Campus of Zunyi Medical University, Zhuhai, China

2 Zhuhai Campus of Zunyi Medical University, Zhuhai, China

*Address all correspondence to: 582578502@qq.com

\section{IntechOpen}

(C) 2020 The Author(s). Licensee IntechOpen. This chapter is distributed under the terms of the Creative Commons Attribution License (http://creativecommons.org/licenses/ by/3.0), which permits unrestricted use, distribution, and reproduction in any medium, provided the original work is properly cited. (cc) BY 


\section{References}

[1] Jaeger MR, Bragasilva J. End-to-end versus end-to-side motor and sensory neurorrhaphy in the repair of the acute muscle denervation. Annals of Plastic Surgery. 2014;67(4):391. DOI: $10.1097 /$ SAP.0b013e3182126816

[2] Haninec P, Filip S, et al. Direct repair (nerve grafting), neurotization, and end-to-side neurorrhaphy in the treatment of brachial plexus injury. Journal of Neurosurgery. 2007;106(3):391-399. DOI: 10.3171/ jns.2007.106.3.391

[3] Viterbo F. Two end-to-side neurorrhaphies and nerve graft with removal of the epineural sheath : Experimental study in rats. British Journal of Plastic Surgery. 1994;47(2):75-80. DOI: 10.1016/0007-1226(94)90162-7

[4] Tarasidis G, Watanabe O, Mackinnon SE, et al. End-to-side neurorraphy: A long-term study of neural regeneration in a rat model. Otolaryngology - Head and Neck Surgery. 1998;119(4):341. DOI: 10.1016/ s0194-5998(98)70074-9

[5] Yu Q, Zhang SH, Wang T, et al. End-to-side neurorrhaphy repairs peripheral nerve injury: Sensory nerve induces motor nerve regeneration. Neural Regeneration Research. 2017;012(010):1703-1707. DOI: $10.4103 / 1673-5374.217350$

[6] Reichert P, Kiełbowicz Z, Kuryszko J, et al. Gait analysis after neurorrhaphy in the brachial plexus. Polish Journal of Veterinary Sciences. 2015. DOI: 10.1515/ pjvs-2015-0047

[7] Stålberg E, Dijk HV, Falck B, et al. Standards for quantification of EMG and neurography. Clinical Neurophysiology. 2019. DOI: 10.1016/j. clinph.2019.05.008
[8] Qiao HY. Comparative Effect of Nerve End-to-Side and Side-to-Side Neurorrhaphy on Prevention and Treatment of Denervated Muscle Atrophy. Shanxi Medical University; 2005

[9] Zhou Z. Experimental study on the prevention of denervated muscle atrophy by side-to-side neurorrhaphy. Journal of Fujian Medical University. 2002;03:292. DOI: CNKI:SUN:FJYD.0.2002-03-025

[10] Rönkkö H, Göransson H, et al. Protective distal side-to-side neurorrhaphy in proximal nerve injury-an experimental study with rats. Acta Neurochirurgica. 2019;161(4):645656. DOI: 10.1007/s00701-019-03835-2

[11] Oberlin C, Béal D,

Leechavengvongs $S$, et al. Nerve transfer to biceps muscle using a part of ulnar nerve for C5-C6 avulsion of the brachial plexus: Anatomical study and report of four cases. The Journal of Hand Surgery. 1994;19(2):232-237. DOI: 10.1016/0363-5023(94)90011-6

[12] Gu Y, Zhang G, Chen D, et al. Seventh cervical nerve root transfer from the contralateral healthy side for treatment of brachial plexus root avulsion. The Journal of Hand Surgery. 1992;17(5):518-521. DOI: 10.1016/ S0266-7681(05) 80235-9

[13] Battiston B, Titolo P, Ciclamini D, et al. Peripheral nerve defects:

Overviews of practice in Europe. Hand Clinics. 2017;33(3):545. DOI: 10.1016/j. hcl.2017.04.005

[14] Gordon T, Borschel GH. The use of the rat as a model for studying peripheral nerve regeneration and sprouting after complete and partial nerve injuries. Experimental Neurology. 2016;287(3):331-347. DOI: 10.1016/ s0140-6736(07)60193-7 
[15] Hood B, Levene HB, Levi AD.

Transplantation of autologous Schwann cells for the repair of segmental peripheral nerve defects. Neurosurgical Focus. 2009;26(2):E4. DOI: 10.3171/ FOC.2009.26.2.E4

[16] Lassner F, Becker M, Berger A, et al. Degeneration and regeneration in nerve autografts and allografts. Microsurgery. 1995;16(1):4-8. DOI: $10.1002 /$ micr.1920160104

[17] Arthur-Farraj PJ, Morgan CC, Adamowicz M, et al. Changes in the coding and non-coding transcriptome and DNA methylome that define the Schwann cell repair phenotype after nerve injury. Cell Reports. 2017;20(11):2719-2734. DOI: 10.1016/j. celrep.2017.08.064

[18] Mackinnon SE, Doolabh VB, Novak CB, et al. Clinical outcome following nerve allograft transplantation. Plastic and Reconstructive Surgery. 2001;107(6):1419-1429. DOI: 10.1097/00006534-200105000-00016

[19] O'Rourke C, AGE D, MurrayDunning C, et al. An allogeneic 'off the shelf' therapeutic strategy for peripheral nerve tissue engineering using clinical grade human neural stem cells. Scientific Reports. 2018;8(1):2951. DOI: $10.1038 / \mathrm{s} 41598-018-20927-8$

[20] Gao Y, Wang Y-l, Kong D, et al. Nerve autografts and tissue-engineered materials for the repair of peripheral nerve injuries: A 5-year bibliometric analysis. Neural Regeneration Research. 2015;6:1003-1008. DOI: 10.4103/1673-5374.158369

[21] Moore AM, Wagner IJ, Fox IK. Principles of nerve repair in complex wounds of the upper extremity. Seminars in Plastic Surgery. 2015;29(1):40-47. DOI: $10.1055 / \mathrm{s}-0035-1544169$
[22] Marasco PD, Kuiken TA. Amputation with median nerve redirection (targeted Reinnervation) reactivates forepaw barrel subfield in rats. Journal of Neuroscience. 2010;30(47):1600816014. DOI: $10.1055 / \mathrm{s}-0035-1544166$

[23] Schiefer M, Tan D, Sidek SM, et al. Sensory feedback by peripheral nerve stimulation improves task performance in individuals with upper limb loss using a myoelectric prosthesis. Journal of Neural Engineering. 2016;13(1):016001. DOI: 10.1088/1741-2560/13/1/016001

[24] Souza JM, Cheesborough JE, $\mathrm{Ko} \mathrm{JH}$, et al. Targeted muscle reinnervation: A novel approach to postamputation neuroma pain. Clinical Orthopaedics and Related Research. 2014;472 (10):2984-2990. DOI: 10.1523/ JNEUROSCI.4044-10.2010

[25] Lowery MM, Stoykov NS, Taflove A, et al. A multiple-layer finite-element model of the surface EMG signal. IEEE Transactions on Biomedical Engineering. 2002;49(5):446-454. DOI: $10.1109 / 10.995683$

[26] Kuiken TA, Marasco PD, Lock BA, et al. Redirection of cutaneous sensation from the hand to the chest skin of human amputees with targeted reinnervation. Proceedings of the National Academy of Sciences of the United States of America. 2007;104(50):20061-20066. DOI: 10.1073/pnas.0706525104

[27] Wijk U, Carlsson I. Forearm amputees' views of prosthesis use and sensory feedback. Journal of Hand Therapy. 2015;28(3):269-278. DOI: 10.1016/j.jht.2015.01.013

[28] Dumanian GA, Ko JH, O’Shaughnessy KD, et al. Targeted reinnervation for transhumeral amputees: Current surgical technique and update on results. Plastic and Reconstructive Surgery. 
2009;124(3):863-869. DOI: 10.1097/ prs.0b013e3181b038c9

[29] Antfolk C, D’Alonzo M, Rosén B, et al. Sensory feedback in upper limb prosthetics. Expert Review of Medical Devices. 2013;10(1):45-54. DOI: 10.1586/erd.12.68

[30] Cheesborough JE, Smith LH, Kuiken TA, et al. Targeted muscle reinnervation and advanced prosthetic arms. Seminars in Plastic Surgery. 2015;29(1):62-72. DOI: 10.1097/ SLA.0000000000003088

[31] Huang H, Zhou P, Li G, et al. An analysis of EMG electrode configuration for targeted muscle reinnervation based neural machine interface. IEEE Transactions on Neural Systems and Rehabilitation Engineering. 2008;16(1):37-45. DOI: $10.1055 / \mathrm{s}-0035-1544166$

[32] Kuiken TA, Miller LA, Lipschutz RD, et al. Targeted reinnervation for enhanced prosthetic arm function in a woman with a proximal amputation: a case study. Lancet (North American Edition). 2007;369(9559):380. DOI: 10.1016/ s0140-6736(07)60193-7

[33] Miller LA, Lipschutz RD, Stubblefield KA, et al. Control of a six degree of freedom prosthetic arm after targeted muscle Reinnervation surgery. Archives of Physical Medicine and Rehabilitation. 2008;89(11):2065. DOI: 10.1016/j.apmr.2008.05.016

[34] Micera S. Staying in touch: Toward the restoration of sensory feedback in hand prostheses using peripheral neural stimulation. IEEE Pulse. 2016;7(3):16-19. DOI: 10.1109/ MPUL.2016.2539760

[35] Sensinger JW, Schultz AE, Kuiken TA. Examination of force discrimination in human upper limb amputees with Reinnervated limb sensation following peripheral nerve transfer. IEEE Transactions on Neural Systems and Rehabilitation Engineering. 2009;17(5):438-444. DOI: 10.1109/ TNSRE.2009.2032640

[36] Yao J, Chen A, Kuiken T, et al. Sensory cortical re-mapping following upper-limb amputation and subsequent targeted reinnervation: A case report. NeuroImage: Clinical. 2015;8:329-336. DOI: 10.1016/j.nicl.2015.01.010

[37] Wall JT, Kaas JH, Sur M, Nelson RJ, Felleman DJ, Merzenich MM. Functional reorganization in somatosensory cortical areas $3 \mathrm{~b}$ and 1 of adult monkeys after median nerve repair: possible relationships to sensory recovery in humans. The Journal of Neuroscience. 1986;6(1):218-233. DOI: 10.1523/ JNEUROSCI.06-01-00218

[38] Osborn L, Kaliki RR, Soares AB, et al. Neuromimetic event based detection for closed-loop tactile feedback control of upper limb prostheses. IEEE Transactions on Haptics. 2016;9(2):196-206. DOI: 10.1109/TOH.2016.2564965

[39] Mioton LM, Dumanian GA, Cheesborough J, et al. targeted muscle Reinnervation successfully treats neuroma pain and phantoms in major limb amputees: A randomized clinical trial. Journal of Neurosurgery. 2018;65(1):86-86. DOI: 10.1093/neuros/ nyy303.117

[40] Zhang MX, Zhang Y, Bian YH, et al. Effect of long-term electroacupuncture stimulation on recovery of sensorimotor function after peripheral nerve anastomosis. Acupuncture in Medicine. 2018;36(3):170-175. DOI: $10.1136 /$ acupmed-2017-011367

[41] Wu J, Lu Y, Hua X, et al. Cortical remodeling after electroacupuncture therapy in peripheral nerve repairing 
model. Brain Research. 2018;1690:61-73. DOI: 10.1016/j.brainres.2018.04.009

[42] He GH, Ruan JW, Zeng YS, et al. Improvement in acupoint selection for acupuncture of nerves surrounding the injury site: Electro-acupuncture with governor vessel with local meridian acupoints. Neural Regeneration Research. 2015;10(1):128-135. DOI: 10.4103/1673-5374.150720

[43] Li XQ, Yu TY, Yu Y, et al. Effects of electroacupuncture on serum myelin basic protein and its antibody in rats with sciatic nerve injury. Journal of Beijing University of Traditional Chinese Medicine. 2015;38(12):842-846. DOI: 10.3969/j. issn.1006-2157.2015.12.010

[44] Hu LN, Guo HD, Tian JX, et al. Electroacupuncture and moxibustion promote regeneration of injured sciatic nerve through Schwann cell proliferation and nerve growth factor secretion. Neural Regeneration Research. 2018;013(003):477-483. DOI: $10.4103 / 1673-5374.228731$

[45] Lu MQ, Yu TY, Yao BB, et al. Effects of tuina therapy on the neural ultrastructure of sciatic nerve injury model rats. Journal of Nanjing University of Traditional Chinese Medicine. 2015;31(4):349-352. DOI: 10.14148/j.issn.1672-0482.2015.0349

[46] Wang XL, Zhu XP, Ji DX, et al. Beneficial effect of traditional Chinese medicine fumigation "bone-healing powder" in postoperative pain and recovery of neurological function of traumatic thoracolumbar spine fractures: A case-control study. Medicine. 2018;97(35):e11983. DOI: 10.1097/MD.0000000000011983

[47] Shao SJ. Acupuncture therapy for peripheral nerve injury. Modern Rehabilitation. 2000;(11). DOI: 10.3321/j.issn:1673-8225.2000.11.006
[48] Zhang MX, Liu YY, Liu JW, et al. The clinical research progress of electroacupuncture treatment of peripheral nerve injury. Bright Chinese Medicine. 2016;31(08):1196. DOI: CNKI:SUN:GMZY.0.2016-08-074

[49] Liu XJ, Cheng Q, Ding F. Experimental study on the regeneration of mouse sciatic nerve by nerve regeneration extract from Achyranthes bidentata. Shizhen Guo Yi Guo Yao. 2009;20(01):16-18. DOI: 10.3969/j. issn.1008-0805.2009.01.008

[50] Liu H, Liu YJ, Liu ZH. Study on the neurotrophic effect of Astragalus membranaceus extract. Journal of Practical Medicine. 2010;26 (14):2480-2482. DOI: CNKI:SUN:SYYZ.0.2010-14-007 



\title{
The Glial Cell of Human Cutaneous Sensory Corpuscles: Origin, Characterization, and Putative Roles
}

\author{
Ramón Cobo, Yolanda García-Mesa, Jorge García-Piqueras, \\ Jorge Feito, José Martín-Cruces, Olivía García-Suárez \\ and José $A$. Vega
}

\begin{abstract}
Sensory corpuscles of human skin are structures located at the peripheral end of the mechanoreceptive neurons and function as low-threshold mechanoreceptors (LTMRs). In its structure, in addition to the axon, there are glial cells, not myelinating, that are organized in different ways according to the morphotype of sensitive corpuscle, forming the so-called laminar cells of Meissner's corpuscles, the laminar cells of the inner core of Pacinian corpuscles, or cells of the inner core in Ruffini's corpuscles. Classically the glial cells of sensory corpuscles have been considered support cells and passive in the process of mechanotransduction. However, the presence of ion channels and synapses-like systems between them and the axon suggests that corpuscular glial cells are actively involved in the transformation of mechanical into electrical impulses. This chapter is an update on the origin, development, cytoarchitecture, and protein profile of glial cells of sensitive corpuscles especially those of human glabrous skin.
\end{abstract}

Keywords: terminal glial cells, lamellar cells, Meissner corpuscles, Pacinian corpuscles, human

\section{Introduction}

The human skin is supplied by sensory nerve fibers which form in the dermis complexes sensory structures known collectively as sensory corpuscles [1]. These sensory formations are connected to nerve fibers originating from intermediate- or large-sized neurons (see for a review [2]) that work as low-threshold mechanoreceptors (LTMRs). LTMRs are classified as $A \beta, A \delta$, or $C$ based on their degree of myelination and action potential conduction velocities $[2,3]$ and functionally fall into two categories: rapidly adapting (RA) and slowly adapting (SA) mechanoreceptors, which each have two variants, type I and type II $[4,5]$. RAI and RAII mechanoreceptors correspond to Meissner and Pacinian corpuscles, respectively; SAI mechanoreceptors are the Merkel cell-neurite complexes, and SAII mechanoreceptors are the dermal Ruffini's corpuscles [1]. This diversity of LTMRs suggests 
differentiated ability to detect and discriminate diverse stimuli in relation to their connectivity to central nervous system nuclei $[1,2,6,7]$.

The peripheral processes of $A \beta$ axons contact in the skin with specialized epithelial cells, i.e., Merkel cells to form Merkel cell-neurite complexes, or with glial Schwann-like cells to form a part of the sensory corpuscles, i.e., Meissner corpuscles, Ruffini's corpuscles, and Pacinian corpuscles [1, 3, 8, 9]. Structurally, the cutaneous sensory corpuscles consists of a dendritic zone (the extreme tip of the peripheral process of an A $\beta$ LTMR), surrounded by nonmyelinating glial cells variably arranged, and both are surrounded by a more or less developed capsule of endoneurial/perineurial cells [9-13]. Filling the spaces among cells, there is a chemically complex extracellular matrix, sometimes organized as a basal lamina [14-17]. So, periaxonic cells that form sensory corpuscles are continuous with the cells of nerve trunks, demonstrating a close relationship between the components of nerves and sensory corpuscles [11].

The peripheral tip of the sensory $A \beta$ axon is always coated by glial cells. These cells constitute a special population of peripheral glial cells denominated terminal glial cells or skin end-organ glia [18], but habitually they are a neglected entity in books and reviews in the topic and are not mentioned among peripheral glial cell types [19]. However, emerging data strongly suggest that glial cells of the sensory corpuscles play key roles in mechanotransduction.

In this review we summarize the current knowledge about the origin and development, cytoarchitecture, immunohistochemical profile, and putative roles of glial cells in sensory corpuscles especially in the genesis of mechanical potential action.

\section{Origin of the sensory corpuscles glial cells}

The glial cells forming a part of the cutaneous sensory corpuscles are regarded as nonmyelinating Schwann-related cells and share some molecular markers. During embryonic development, peripheral glial progenitors originated in the neural crest (NC) cells contact the surface of developing axons and differentiate into Schwann cell precursors (SCPs) [20]. Subsequently, SCPs originate immature Schwann cells (ISCs) that later generate adult peripheral glial cells types, including the glia found in cutaneous sensory end organs [18].

Neural crest boundary cells (NC-BCs) can also generate peripheral glia during embryonic development [21] which in the presence of neuregulins (NRs) are able to differentiate into SCPs [22] and then into ISCs before maturing into myelinating and nonmyelinating Schwann cells [23]. The immediate progeny of NC-BCs, together with other nerve-associated SCPs, migrate along the sensory nerves toward the skin to give rise to highly specialized glial cells (denominated specific sensory nerve fiber-associated glia in the skin) associated to nerve endings in the epidermis although these cells are distinct to the specialized glial subtype found within the cutaneous sensory corpuscles [24]. But according to Etxaniz and coworkers [25], in the skin BC derivatives give rise to at least three glial populations: Schwann cells (mainly nonmyelinating) associated with subcutaneous and dermal nerves and two types of terminal Schwann cells, associated with lanceolate endings or free nerve endings. It can be speculated that the glial cells of sensory corpuscles can derivate of that first type.

Furthermore, NC-derived stem cells are retained postnatally in the skin and peripheral nerves after differentiation the SCPs cells and do not completely lost multipotentiality [26] retaining certain characteristics of NC cells and remaining multipotent $[27,28]$. 
In view of the diverse possible origins of the glial cells of sensory corpuscles, it is not possible to know exactly the cells from which they come. Probably these cells are a consequence of combinatory distinct molecular signatures and local factors during development [18].

\section{Development of the sensory corpuscles glial cells}

Sensory axons are critical inducing the development of sensory corpuscles, and reciprocal interactions between axons and target cells, especially peripheral glial cells, seem to initiate their morphogenesis [29]. SCPs comigrate with growing axons in peripheral nerves upon axonal signals such as NRG-1 [30,31].

Some of the molecules that interplay those axon-peripheral glial cells relationships in developing sensory corpuscles are now also known. The neurotrophin (NT) family of growth factors is involved in the development of mechanoreceptors controlling the development of mechanosensory neurons. Mice lacking TrkB and its ligand brain-derived neurotrophic factor (BDNF), but not NT-4, do not develop Meissner-like corpuscles [32-34], whereas overexpression of BDNF [35] and NT-4 [36] leads to an increase in the size and density of those corpuscles. The role of NTs in regulating development of Pacinian corpuscles is more complex and controversial since multiple NT-Trk signals participate, resulting in a reduction of the number of Pacinian corpuscles in mice deficient for BDNF and NT-3 and TrkA and TrkB [37]. However, Pacinian corpuscles of postnatal TrkB-deficient mice were found largely normal [33, 38]. ER81 is also present in developing murine Pacinian corpuscles [39], and in the absence of this transcription factor, Pacinian corpuscles do not form because their afferents do not survive. NRG-1 interacts with ErbB2/ErbB3 receptors on Schwann cell lineage and is broadly involved in Schwann cell development [40]. Recently, it has been demonstrated that a RET-ER81-NRG-1 signaling pathway promotes axon communication with nonmyelinating Schwann cells. The glial cells forming inner core of murine Pacinian corpuscles display NRG receptors erbB2, erbB3, and erbB4, whereas the central axon is immunoreactive for NRG-1 and ablating Ret and Nrg-1 in mechanosensory neurons results in the absence of Pacinian corpuscles, while Meissner's corpuscles were unaffected [41-43]. Interestingly, the dependence of the corpuscular glial cells from the axons continues during adult life at least for the expression of some antigens. After denervation, glial cells of Meissner-like corpuscles lack some specific markers [44, 45] and strongly decrease the expression of TrkA [46], and the glial cells forming the inner core of Pacinian corpuscles undergo apoptotic death that can be prevented by administration of glial growth factor 2 [47].

In addition to the axon-glial cells interactions, probably local molecules participate in the development of sensory corpuscles including growth factors $[48,49]$, $\beta$-arrestin-1 [50], semaphorins [49, 51, 52], ankyrin-B [53], and also mechanical signals [54].

NC cells, SCPs, and ISCs share some markers (neuregulin receptors ErbB2 and ErbB3, L1, nestin, vimentin). Two markers used for labeling of mature Schwann cells, i.e., S100 protein and vimentin, are also present in ISCs but are absent or expressed at much lower levels in PSCs [30,55]. Using these proteins as ISCs or mature Schwann and Schwann-related cell markers, we have determined the timetable of the development of sensory corpuscles. In murine Meissner-like corpuscles start to express immunoreactivity for S100 protein by postnatal day 7 (Pd7), vimentin by $\mathrm{Pd} 12$, and $\mathrm{p} 75^{\mathrm{LNGFR}}$ (a marker for peripheral glia too) transitory from Pd7 to Pd19. Pacinian corpuscles show S100 protein in the inner core at Pd7, whereas vimentin starts expression at Pd19 and later [56]. In human, the first evidence 
of Pacinian corpuscles was at 13 weeks of estimated gestational age (wega). At this time, and until 16 wega, the S100 protein positive from one or two layers of rounded cells then (16-18 wega) become flattened and show lamellar organization, thus originating a primitive inner core. Thereafter, between 20 and 24 wega, the S100 protein-positive cells emitted cytoplasmic expansions that invaded the outer region of the corpuscle forming a network. In the period between 24 and 36 wega, the edge of the inner core was still not totally defined until the fourth month of life, when the lamellae forming it become strongly packed and the inner core clefts are clearly distinguished. During development of Pacinian corpuscles, expression of vimentin started shortly later than that of S100P and did not vary along lifespan. On the other hand, at 23 wega hook-shaped axonal profiles are identified in the dermal papillae, but S100P-positive cells reach this place at 33 wega. By 36-40 wega, the S100 protein-positive lamellar cells of incipient Meissner's corpuscles can become progressively flattened, and around these cells also express vimentin, but their definite and typical arrangement occurs in the first weeks of life. Along the first semester of life, the S100P-positive cells become definitively flattened, reaching the adult morphology around 8 months [57].

\section{Cytoarchitecture of glial cells in the different morphotypes of cutaneous sensory corpuscles}

The arrangement of terminal glial cells in the different morphotypes of sensory corpuscles varies from one to another, either irregularly (Krause and Ruffini's corpuscles), regularly (Meissner corpuscles), or forming the lamellar system of the inner core (Pacinian corpuscles) [8,9].

In Meissner's corpuscles, the terminal glial cells are currently denominated lamellar cells.

The organization of the lamellar has a typical flattened appearance due to the horizontal lamelation that form stacks of lamellae separated by axon branches [8, 58]. Habitually the nuclei of the cells are at the periphery of the corpuscle and are total or partially covered by a CD34-positive capsule of endoneurial origin [13] (Figure 1).

The inner core is the zone of Pacinian corpuscles that lies between the axon and the intermediate layer and consists of tightly packed lamellae of peripheral glial cells which are diversely organized in the preterminal, terminal, and ultraterminal zones of the corpuscle. In the preterminal zone, the axon is still covered by the myelin sheath; the terminal zone characteristically has a bilateral symmetric organization; and in the ultraterminal zone, glial cells lose bilaterally. The flattened lamellar cells forming the terminal zone of the inner core have the nuclei lying in the outer core of the inner core itself. The lamellar cells project processes from the outer margin into the inner core, and flattening give the appearance of concentric layers of lamellae arranged in bilateral symmetry, hemilamellae, and the tips of the lamellae are separated by two clefs that run along the entire length of the axon until the ultraterminal zone. Interestingly the lamellae of the inner core have numerous gap (tight) junctions as well as desmosome-like junctions (see for a review [8, 9, 59]) (Figure 2).

Regarding Ruffini's corpuscles, the glial cells forming the inner core have an irregular distribution within the capsule with variable relationship with the dendritic zone of the axon tip [8] (Figure 3).

The terminal glial cells forming the inner core of Pacinian corpuscles and the lamellar cells of the Meissner ones are covered by a basal lamina, and around there is a complex extracellular matrix, whose composition in now rather well known [14-17, 60]. 
The Glial Cell of Human Cutaneous Sensory Corpuscles: Origin, Characterization... DOI: http://dx.doi.org/10.5772/intechopen.91815
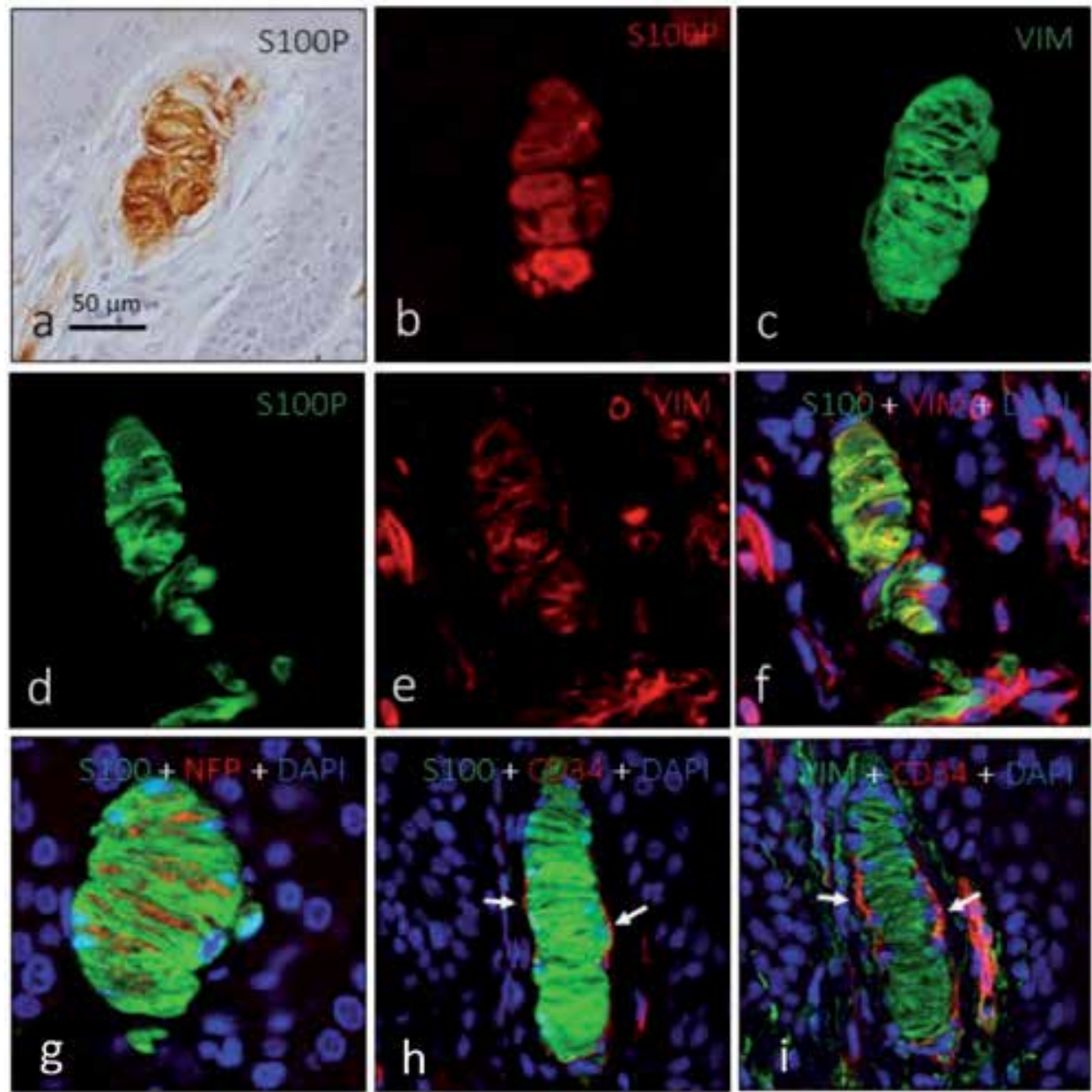

Figure 1.

Immunohistochemical localizacion of S10o protein (S10oP; $a, b, g, h$ ), vimentin (VIM; $c, e, i$ ), neurofilament proteins (red in $g$ ) and $C_{34}$ (red in $h, i$ ) in Meissner'w corpuscles. The lamellar cells display intense and selective cytoplasmic S10oP and VIM which are colocalized $(d-f)$. The lamellar are in close contact with the axon (red fluorescence in $g$ ) and an endoeneurial capsule (red fluorescence in $h$ and $i$ ).

\section{Immunohistochemical profile}

\subsection{Cytoskeletal proteins and general markers}

Glial fibrillary acidic protein (GFAP) is theoretically the intermediate filament protein filling the cytoplasm of Schwann and Schwann-related cells forming cutaneous sensory corpuscles. However, most authors consider that peripheral glial cells express vimentin as the main intermediate filament protein [61]. In agreement with this assumption, GFAP was always absent from rat [62] and human [63] cutaneous sensory corpuscles. In contrast, the cytoplasm of the peripheral glial cells of sensory corpuscles expresses vimentin [62-64]. However, GFAP immunoreactivity was detected in the innermost lamellae of the inner core of feline Pacinian corpuscles [65] and human pancreatic Pacinian corpuscles [66].

The $\mathrm{Ca}^{2+}$-binding proteins represent one of the physiological mechanisms for maintaining intracellular $\mathrm{Ca}^{2+}$ homeostasis [67]. Some of these proteins have been 

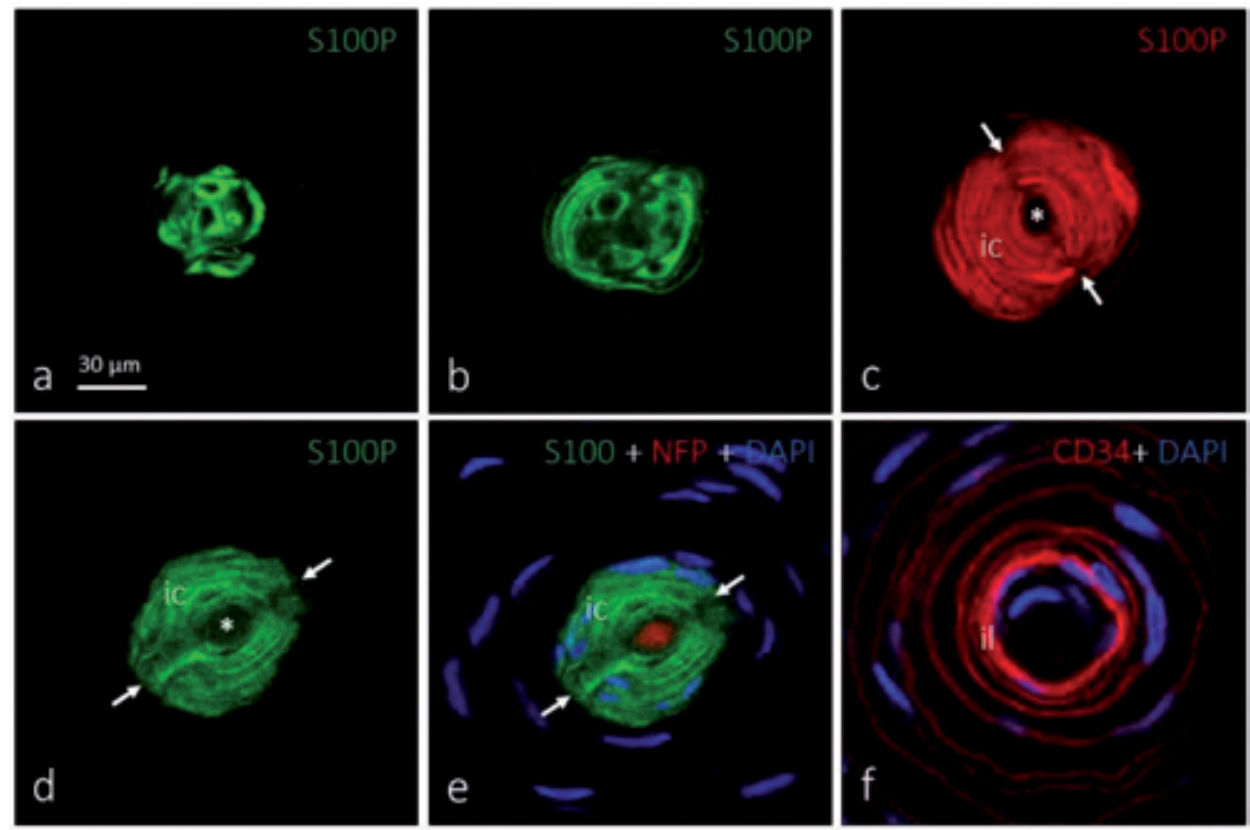

Figure 2.

Immunohistochemical localization of S10o protein (S10o; a-e), neurofilament protein (NFP; red fluorescence in e) and $C_{34}$ (red fluorescence in $f$ ). The glial cells forming the central zone of the inner core are characteristically arranged into two symmetrical hemilamellar systems separated by clefts (arrows in c-e) whereas in the ultraterminal zone of the inner core are irregularly disposed $(a, b)$. The inner most lamellae of the inner core is in close contact with the axon (e) while de outer most lamellae is closely related with the so-called intermediate layer. ic: inner core; il: intermediate layer; asterisks indicate the zone occupied by the axon.
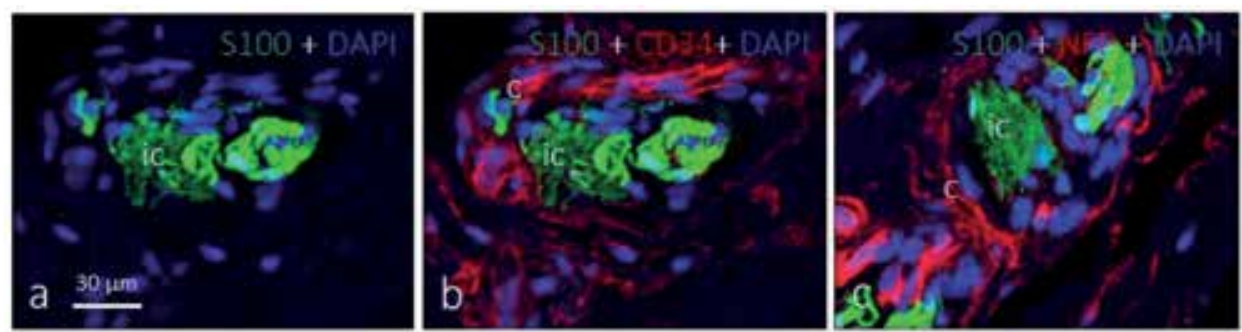

Figure 3.

Immunohistochemical localization of S10o protein (a-c) and CD34 in human cutaneous Ruffini's corpuscles. The glial cells are irregularly arranged and display a strong S10oP immunofluorescence $(a-c)$. The capsule of these corpuscles is $C D 34$ positive suggesting and endoneurial origin $(b, c)$. c: capsule; ic: inner core.

found in the glial cells of cutaneous sensory corpuscles, most of them belonging to the so-called "EF-hand" family. They include S100 protein, calbindin D28k, parvalbumin, and calretinin (sensory corpuscles) [10, 68, 69].

\subsection{Growth factors and growth factor receptors}

As far as we know, the only growth factors detected in the glial cells of sensory corpuscles are TGF- $\beta$ in the lamellar cells of rat Meissner corpuscles [70] and BDNF in the inner core cells of digital Pacinian corpuscles of Macaca fascicularis [71]. However, the glial cells of sensory corpuscles display a wide range of receptors for different growth factors. Epidermal growth factor receptor was detected in the lamellar and the inner core cells of human Meissner and Pacinian corpuscles, 
respectively [72], as well as low- and high-affinity receptors for NTs including p75 ${ }^{\mathrm{NNR}}$ [73-76], TrkA [77], and TrkB [78, 79]. TGF- $\beta$ receptors RI and RII are also present in the inner core lamellar cells of cat Pacinian corpuscles and the lamellar cells of Meissner corpuscles [70].

\section{Are the corpuscular glial cells accessory cells or active cells?}

\subsection{The GABA-ergic/glutamatergic system in Pacinian corpuscles}

The inner core of Pacinian corpuscles expresses immunoreactivity for glutamate receptors, vesicular-glutamate transporters, and the synaptic proteins synaptobrevin (VAMP2) and SNAP-23. Moreover, inner core cells release neurotransmitters (glutamate, GABA) when they are stimulated by glutamate, ATP, or even by mechanical motion [80]. This implies "synaptic-like" interaction between the axon and the glial cells of Pacinian corpuscles. This hypothesis, postulated by Pawson and co-workers [80,81], argues that: "action potentials in response to dynamic stimuli are due to depolarization of the axon by cations entering mechano-gated channels that are opened due to mechanical motion; however, action potentials in the static portion of the Pacinian corpuscle rapidly adapting response are due to glutamatergic excitation, which are then inhibited by GABA released from the modified Schwann cells of the inner core." These results suggest that in the Pacinian corpuscles, GABA emanating from the capsule inhibits glutamate excitation (stemming either from the neurite itself or from the capsule), leading to a glial-neuronal "mechanochemical," rather than solely mechanical, RA response to sustained pressure. These elegant and attractive results should be confirmed in other mechanoreceptors and in different vertebrate species.

\subsection{Ion channels}

In the past, investigators proposed that the response of sensory corpuscles could be explained entirely by the mechanical properties periaxonic cells, especially the capsule. Then the discovery that some ion channels are gated by mechanical forces (mechanosensitive ion channels) suggests that mechanotransduction occurs through the activation of ion channels along the somatosensory neurons that reach the skin. The opening of these channels consent the entry of ions within the axon to produce the mechanotransduction [82-86]. Thus, deformations in the membrane of different cells that form the mechanoreceptors (i.e., axon, glial cells, and endoneurial and/or perineurial fibroblast) trigger the opening of mechanosensitive ion channels that transduce mechanical energy into electrical activity. Consistently, the cells forming the mechanoreceptors are thought to express ion channels activated by force or displacement to act as mechanodetectors and/or mechanotransducers. Thus, mechanotransduction can be defined as the conversion of a mechanical stimulus into an electrical signal, and in the sensory corpuscles, the first step of mechanotransduction takes place $[87,88]$.

Numerous types of mechanically gated ion channels were found in vertebrate sensory corpuscles, but most of them were localized in the axon. Nevertheless, evidence exists that some of them are also present in the glial cells. The inner core of murine [89] and human [90] Pacinian corpuscles displays immunoreactivity for ASIC2, a member of the acid-sensing ion channels included in the degenerin/ epithelial $\mathrm{Na}^{+}$channel superfamily. In a subpopulation of human Meissner corpuscles, the lamellar cells also show ASIC2 immunoreactivity [91]. Some members of the superfamily of the transient receptor potential (TRP) ion channels have been 
detected in glial cells of sensory corpuscles. TRPV4 was detected in the lamellar cells of human Meissner corpuscles [92]. On the other hand, voltage-sensitive $\mathrm{Na}^{+}$ channels ( $\alpha$-subunit type I and type II voltage-gated $\mathrm{Na}^{+}$channel) present in the inner core lamellae and the axon might participate in both transduction and action potential generation [93].

Based on the above data in addition to the hypothesis of voltage-gated and nonvoltage-gated channels, a possible classical neurotransmission cannot be excluded for the genesis of the action potential in sensory corpuscles. In the process of touch sensation, a mechanical stimulus is converted into electrical activity in peripheral sensory neurons, and this conversion may occur through the activation of ion channels that gate in response to mechanical stimuli.

\section{Concluding remarks}

The glial cells of the sensory corpuscles form a glial subpopulation, highly differentiated and with important functions in the mechanotransduction process that has been repeatedly forgotten in studies on peripheral glia. Its origin is not known exactly, and they could come from cells of the neural crest or the boundary cap cells. However, the chronology of its arrival and organization within the corpuscles has recently been established, as well as their interdependence with sensory axons. In the last decade, some mechano-gated ion channels have been discovered in corpuscular glial cells. This fact associated with the demonstration of a GABA-ergic/ glutamatergic neurotransmission system in the Pacinian corpuscles suggests that the glial cells of the sensory corpuscles are not support cells but have an active role in the mechanotransduction process. Nevertheless, whether or not this occurs only in Pacinian corpuscles or in all sensory corpuscles remains to be demonstrated.

\section{Acknowledgements}

This study was supported in part by a grant from Gerencia Regional de Salud de Castilla y León (GRS 1615/A/17). YG-M was supported by a grant "Severo Ochoa” from the Government of the Principality of Asturias (Ref. BP17-044). The authors thank Dr. Marta Guervos (Servicios Comunes de Investigación, Microscopia Confocal, Universidad de Oviedo) and Marta Sánchez-Pitiot (Grupo de Histopatología Molecular, Instituto Universitario de Oncología del Principado de Asturias) for their technical assistance. 
The Glial Cell of Human Cutaneous Sensory Corpuscles: Origin, Characterization...

DOI: http://dx.doi.org/10.5772/intechopen.91815

\section{Author details}

Ramón Cobo ${ }^{1}$, Yolanda García-Mesa ${ }^{1}$, Jorge García-Piqueras ${ }^{1}$, Jorge Feito ${ }^{1,2,3}$, José Martín-Cruces ${ }^{1}$, Olivía García-Suárez ${ }^{1}$ and José $\mathrm{A}$. Vega ${ }^{1,4 *}$

1 Departamento de Morfología y Biología Celular, Grupo SINPOS, Universidad de Oviedo, Oviedo, Spain

2 Departamento de Anatomía e Histología Humanas, Universidad de Salamanca, Spain

3 Servicio de Anatomía Patológica, Complejo Hospitalario, Universitario de Salamanca, Salamanca, Spain

4 Facultad de Ciencias de la Salud, Universidad Autónoma, Santiago, Chile

*Address all correspondence to: javega@uniovi.es

\section{IntechOpen}

(C) 2020 The Author(s). Licensee IntechOpen. This chapter is distributed under the terms of the Creative Commons Attribution License (http://creativecommons.org/licenses/ by/3.0), which permits unrestricted use, distribution, and reproduction in any medium, provided the original work is properly cited. (cc) BY 


\section{References}

[1] Zimmerman A, Bai L, Ginty DD. The gentle touch receptors of mammalian skin. Science. 2014;346:950-954

[2] Abraira VE, Ginty DD. The sensory neurons of touch. Neuron. 2013;79:618-639

[3] Rice FL, Albrecht PJ. Cutaneous mechanisms of tactile perception: Morphological and chemical organization of the innervation to the skin. In: Somatosensation GE, Kaas JA, editors. The Senses: A Comprehensive Reference. Vol. 6. Amsterdam: Elsevier; 2008. pp. 1-31

[4] Jones LA, Smith AM. Tactile sensory system: Encoding from the periphery to the cortex. Wiley Interdisciplinary Reviews. Systems Biology and Medicine. 2014;6:279-287

[5] Olson W, Dong P, Fleming M, Luo W. The specification and wiring of mammalian cutaneous lowthreshold mechanoreceptors. Wiley Interdisciplinary Reviews: Developmental Biology. 2016;5:389-404

[6] Oaklander AL, Siegel SM. Cutaneous innervation: Form and function. Journal of the American Academy of Dermatology. 2006;53:1027-1037

[7] McGlone F, Reilly D. The cutaneous sensory system. Neuroscience and Biobehavioral Reviews. 2010;34:148-159

[8] Munger BL, Ide C. The structure and function of cutaneous sensory receptors. Archives of Histology and Cytology. 1988;51:1-34

[9] Zelena J. Nerves and

Mechanoreceptors: The Role of Innervation in the Development and Maintenance of Mammalian Mechanoreceptors. New York: Chapman \& Hall; 1994
[10] Vega JA, Haro JJ, Del Valle ME. Immunohistochemistry of human cutaneous Meissner and Pacinian corpuscles. Microscopy Research and Technique. 1996;34:351-361

[11] Vega JA, García-Suárez O, Montaño JA, Pardo B, Cobo JM. The Meissner and Pacinian sensory corpuscles revisited: New data from the last decade. Microscopy Research and Technique. 2009;72:299-309

[12] García-Piqueras J, García-Suárez O, Rodríguez-González MC, Cobo JL, Cabo R, VegaJA, et al. Endoneurial-CD34 positive cells define an intermediate layer in human digital Pacinian corpuscles. Annals of Anatomy. 2017;211:55-60

[13] García-Piqueras J, Cobo R, Cárcaba L, García-Mesa Y, FeitoJ, CoboJ, et al. The capsule of human Meissner corpuscles: Immunohistochemical evidence. Journal of Anatomy. 2020. DOI: 10.1111/joa.13139

[14] Dubový P, Bednárová J. The extracellular matrix of rat pacinian corpuscles: An analysis of its fine structure. Anatomy and Embryology. 1999;200:615-623

[15] García-Piqueras J, Carcaba L, García-Mesa Y, Feito J, García B, Viña E, et al. Chondroitin sulfate in human cutaneous Meissner and Pacinian sensory corpuscles. Anatomical Record (Hoboken, N.J.). 2019;302:325-331

[16] García-Piqueras J, García-Mesa Y, Feito J, García B, Quiros LM, Martín-Biedma B, et al. Class I and class II small leucine-rich proteoglycans in human cutaneous pacinian corpuscles. Annals of Anatomy. 2019;224:62-72

[17] García-Piqueras J, García-Suárez O, García-Mesa Y, García-Fernandez B, Quirós LM, Cobo R, et al. Heparan 
The Glial Cell of Human Cutaneous Sensory Corpuscles: Origin, Characterization...

sulfate in human cutaneous Meissner's and Pacinian corpuscles. Anatomical Record (Hoboken, N.J.). 2020. DOI: 10.1002/ar.24328

[18] Kastriti ME, Adameyko I. Specification, plasticity and evolutionary origin of peripheral glial cells. Current Opinion in Neurobiology. 2017;47:196-202

[19] Jessen KR, Mirsky R. Schwann cell precursors; multipotent glial cells in embryonic nerves. Frontiers in Molecular Neuroscience. 2019;12:69

[20] Furlan A, Adameyko I. Schwann cell precursor: A neural crest cell in disguise? Developmental Biology. 2018;444(Suppl 1):S25-S35

[21] Maro GS, Vermeren M, Voiculescu O, Melton L, Cohen J, Charnay P, et al. Neural crest boundary cap cells constitute a source of neuronal and glial cells of the PNS. Nature Neuroscience. 2004;7:930-938

[22] Petersen J, Adameyko I. Nerveassociated neural crest: Peripheral glial cells generate multiple fates in the body. Current Opinion in Genetics \& Development. 2017;45:10-14

[23] Aquino JB, Hjerling-Leffler J, Koltzenburg M, Edlund T, Villar MJ, Ernfors P. In vitro and in vivo differentiation of boundary cap neural crest stem cells into mature Schwann cells. Experimental Neurology.

2006;198:438-449

\section{[24] Gresset A, Coulpier F,} Gerschenfeld G, Jourdon A, Matesic G, Richard L, et al. Boundary caps give rise to neurogenic stem cells and terminal glia in the skin. Stem Cell Reports. 2015;5:278-290

[25] Etxaniz U, Pérez-San Vicente A, Gago-López N, García-Dominguez M, Iribar H, Aduriz A, et al. Neuralcompetent cells of adult human dermis belong to the Schwann lineage. Stem Cell Reports. 2014;3:774-788

[26] Dyachuk V, Furlan A, Shahidi MK, Giovenco M, Kaukua N, Konstantinidou C, et al. Neurodevelopment. Parasympathetic neurons originate from nerve-associated peripheral glial progenitors. Science. 2014;345:82-87

[27] Widera D, Heimann P, Zander C, Imielski Y, Heidbreder M, Heilemann M, et al. Schwann cells can be reprogrammed to multipotency by culture. Stem Cells and Development. 2011;20:2053-2064

[28] Calavia M, Viña E, MenéndezGonzález M, López-Muñiz A, Alonso-Guervós M, Cobo J, et al. Evidence of nestin-positive cells in the human cutaneous Meissner and Pacinian corpuscles. CNS \& Neurological Disorders Drug Targets. 2012;11:869-877

[29] Saxod R. Ontogeny of the cutaneous sensory organs. Microscopy Research and Technique. 1996;34:313-333

[30] Jessen KR, Mirsky R. The origin and development of glial cells in peripheral nerves. Nature Reviews. Neuroscience. 2005;6:671-682

[31] Nave KA, Salzer JL. Axonal regulation of myelination by neuregulin 1. Current Opinion in Neurobiology. 2006;16:492-500

[32] IchikawaH,MatsuoS,Silos-SantiagoI, Sugimoto T. Developmental dependency of Meissner corpuscles on trkB but not trkA or trkC. Neuroreport. 2000;11:259-262

[33] González-Martínez T, Germanà GP, Monjil DF, Silos-Santiago I, de Carlos F, Germanà G, et al. Absence of Meissner corpuscles in the digital pads of mice lacking functional TrkB. Brain Research. 2004;1002:120-128 
[34] González-Martínez T, Fariñas I, Del Valle ME, Feito J, Germanà G, Cobo J, et al. BDNF, but not NT-4, is necessary for normal development of Meissner corpuscles. Neuroscience Letters. 2005;377:12-15

[35] LeMaster AM, Krimm RF, Davis BM, Noel T, Forbes ME, Johnson JE, et al. Overexpression of brain-derived neurotrophic factor enhances sensory innervation and selectively increases neuron number. The Journal of Neuroscience. 1999;19:5919-5931

[36] Krimm RF, Davis BM, Noel T, Albers KM. Overexpression of neurotrophin 4 in skin enhances myelinated sensory endings but does not influence sensory neuron number. The Journal of Comparative Neurology. 2006;498:455-465

[37] Sedý J, Szeder V, Walro JM, Ren ZG, Nanka O, Tessarollo L, et al. Pacinian corpuscle development involves multiple Trk signaling pathways. Developmental Dynamics. 2004;231:551-563

[38] Pérez-Piñera P, García-Suarez O, Germanà $\mathrm{A}$, Díaz-Esnal $\mathrm{B}$, de Carlos $\mathrm{F}$, Silos-Santiago I, et al. Characterization of sensory deficits in TrkB knockout mice. Neuroscience Letters.

2008;433:43-47

[39] Sedý J, Tseng S, Walro JM, Grim M, Kucera J. ETS transcription factor ER81 is required for the Pacinian corpuscle development. Developmental Dynamics. 2006;235:1081-1089

[40] Birchmeier C, Nave KA. Neuregulin-1, a key axonal signal that drives Schwann cell growth and differentiation. Glia. 2008;56:1491-1497

[41] Luo W, Enomoto H, Rice FL, Milbrandt J, Ginty DD. Molecular identification of rapidly adapting mechanoreceptors and their developmental dependence on ret signaling. Neuron. 2009;64:841-856

[42] Fleming MS, Li JJ, Ramos D, Li T, Talmage DA, Abe SI, et al. A RET-ER81NRG1 signaling pathway drives the development of Pacinian corpuscles. The Journal of Neuroscience.

2016;36:10337-10355

[43] González-Martínez T, Germana A, Catania S, Cobo T, Ochoa-Erena FJ, de Carlos F, et al. Postnatal developmental changes in the expression of ErbB receptors in murine Pacinian cospucles. Neuroscience Letters. 2007;420:90-95

[44] Del Valle ME, Cabal A, AlvarezMendez JC, Calzada B, Haro JJ, Collier W, et al. Effect of denervation on lamellar cells of Meissner-like sensory corpuscles of the rat. An immunohistochemical study. Cellular and Molecular Biology. 1993;39:801-807

[45] Albuerne M, López S, Naves FJ, Martinez-Almagro A, Represa J, Vega JA. S100 $\alpha$ and S100 $\beta$ proteins in human cutaneous sensory corpuscles: Effects of nerve and spinal cord injury. The Anatomical Record. 1998;251:351-359

[46] Márquez J, Pérez-Pérez M, Naves FJ, Vega JA. Effect of spinal cord and peripheral nerve injury on human cutaneous sensory corpuscles. An immunohistochemical study. Journal of the Peripheral Nervous System. 1997;2:49-59

[47] Kopp DM, Trachtenberg JT, Thompson WJ. Glial growth factor rescues Schwann cells of mechanoreceptors from denervationinduced apoptosis. The Journal of Neuroscience. 1997;17:6697-6706

[48] Schecterson LC, Bothwell M. Novel roles for neurotrophins are suggested by BDNF and NT-3 mRNA expression in developing neurons. Neuron. 1992;9:449-466 
The Glial Cell of Human Cutaneous Sensory Corpuscles: Origin, Characterization... DOI: http://dx.doi.org/10.5772/intechopen.91815

[49] Dontchev VD, Letourneau PC. Growth cones integrate signaling from multiple guidance cues. The Journal of Histochemistry and Cytochemistry. 2003;51:435-444

[50] Komori N, Neal J, Cain SD, Logan J, Wirsig C, Miller KE. Presence of betaarrestin-1 immunoreactivity in the cutaneous nerve fibers of rat glabrous skin. Brain Research. 2003;988:121-129

[51] Curley JL, Catig GC, HornRanney EL, Moore MJ. Sensory axon guidance with semaphorin $6 \mathrm{~A}$ and nerve growth factor in a biomimetic choice point model. Biofabrication. 2014;6:035026

[52] McCormick AM, Jarmusik NA, Leipzig ND. Co-immobilization of semaphorin $3 \mathrm{~A}$ and nerve growth factor to guide and pattern axons. Acta Biomaterialia. 2015;28:33-44

[53] Engelhardt M, Vorwald S, Sobotzik JM, Bennett V, Schultz C. Ankyrin-B structurally defines terminal microdomains of peripheral somatosensory axons. Brain Structure \& Function. 2013;218:1005-1016

[54] Koser DE, Thompson AJ, Foster SK, Dwivedy A, Pillai EK, Sheridan GK, et al. Mechanosensing is critical for axon growth in the developing brain. Nature Neuroscience. 2016;19:1592-1598

[55] Aquino JB, Sierra R. Schwann cell precursors in health and disease. Glia. 2018;66:465-476

[56] Albuerne M, De Lavallina J, Esteban I, Naves FJ, Silos-Santiago I, Vega JA. Development of Meissnerlike and Pacinian sensory corpuscles in the mouse demonstrated with specific markers for corpuscular constituents. The Anatomical Record. 2000;258:235-242

[57] Feito J, García-Suárez O, García-Piqueras J, García-Mesa Y,
Pérez-Sánchez A, Suazo I, et al. The development of human digital Meissner's and Pacinian corpuscles. Annals of Anatomy. 2018;219:8-24

[58] Vega JA, López-Muñiz A, Calavia MG, García-Suárez O, Cobo J, Otero J, et al. Clinical implication of Meissner's corpuscles. CNS \& Neurological Disorders Drug Targets. 2012;11:856-868

[59] Bell J, Bolanowski S, Holmes MH. The structure and function of Pacinian corpuscles: A review. Progress in Neurobiology. 1994;42:79-128

[60] Vega JA, Esteban I, Naves FJ, del Valle ME, Malinovsky L. Immunohistochemical localization of laminin and type IV collagen in human cutaneous sensory nerve formations. Anatomy and Embryology. 1995;191:33-39

[61] Bianchini D, De Martini I, Cadoni A, Zicca A, Tabaton M, Schenone A, et al. GFAP expression of human Schwann cells in tissue culture. Brain Research. 1992;570:209-217

[62] Vega JA, Malinovsky L, Del Valle ME, Hernandez LC, Dubovy P, Perez-Casas A. Immunohistochemical study of sensory formations from the glabrous skin of the rat. Zeitschrift fur mikroskopisch-anatomische Forschung. 1990;104:779-787

[63] Haro JJ, Vega JA, Del Valle ME, Calzada B, Zaccheo D, Malinovsky L. Immunohistochemical study of sensory nerve formations in the human glabrous skin. European Journal of Morphology. 1991;29:271-284

[64] Vega JA, Del Valle ME, Haro JJ, Naves FJ, Calzada B, Uribelarrea R. The inner-core, outer-core and capsule of the human Pacinian corpuscles: An immunohistochemical study. European Journal of Morphology. 1994;32:11-18 
[65] Vega JA, Dubovy P, del ValleSoto ME, Hernandez LC, Perez-Casas A, Malinovsky L. Immunohistochemical study of Pacinian corpuscles using monoclonal antibodies for neurofilament protein, glial fibrillary acidic protein and S-100 protein. Cellular and Molecular Biology. 1989;35:627-633

[66] García-Suárez O, Calavia MG, Pérez-Moltó FJ, Alvarez-Abad C, Pérez-Piñera P, Cobo JM, et al. Immunohistochemical profile of human pancreatic pacinian corpuscles. Pancreas. 2010;39:403-410

[67] Andressen C, Bliimke I, Celio MR. Calcium-binding proteins: Selective markers of nerve cells. Cell and Tissue Research. 1993;271:181-208

[68] Del Valle ME, Vazquez E, Naves FJ, Represa JJ, Malinovsky L, Vega JA. Immunohistochemical localization of calcium-binding proteins in the human cutaneous sensory corpuscles. Neuroscience Letters. 1994;168:247-250

[69] Gonzalez-Martinez T, PerezPiñera P, Díaz-Esnal B, Vega JA. S-100 proteins in the human peripheral nervous system. Microscopy Research and Technique. 2003;60:633-638

[70] Stark B, Carlstedt T, Risling M. Distribution of TGF- $\beta$, the TGF- $\beta$ type I receptor and the R-II receptor in peripheral nerves and mechanoreceptors; observations on changes after traumatic injury. Brain Research. 2001;913:47-56

[71] Cabo R, Alonso P, San José I, Vázquez G, Pastor JF, Germanà $A$, et al. Brain-derived neurotrophic factor and its receptor $\operatorname{TrkB}$ are present, but segregated, within mature cutaneous Pacinian corpuscles of Macaca fascicularis. Anatomical record (Hoboken, N.J.). 2015;298:624-629
[72] Vega JA, Vazquez E, Naves FJ, Calzada B, Del Valle ME, Represa JJ. Expression of epidermal growth factor receptor (EGFr) immunoreactivity in human cutaneous nerves and sensory corpuscles. The Anatomical Record. 1994;240:125-130

[73] Ribeiro da Silva A, Kenigsberg RL, Cuello AC. Light and electron microscopic distribution of nerve growth factor receptor-like immunoreactivity in the skin of the rat lower lip. Neuroscience. 1991;43:631-646

[74] Vega JA, Del Valle ME, Haro JJ, Calzada B, Suarez-Garnacho S, Malinovsky L. Nerve growth factor receptor immunoreactivity in Meissner and Pacinian corpuscles of the human digital skin. The Anatomical Record. 1993;236:730-736

[75] Vega JA, Haro JJ, De Lamo A, Ordieres M, Del Valle ME, Calzada B. Distribution of low affinity nerve growth factor receptors (NGFr) immunoreactivity in the human digital skin. European Journal of Dermatology. 1993;2:509-516

[76] Schatteman GC, Langer T, Lanahn AA, Bothwell MA. Distribution of $75-\mathrm{kD}$ low affinity nerve growth factor receptor in the primate peripheral nervous system. Somatosensory \& Motor Research. 1993;10:415-432

[77] Vega JA, Vazquez E, Naves FJ, Del Valle ME, Calzada B, Represa JJ. Immunohistochemical localization of the high affinity NGF receptor (p140kDa-trkA) in adult dorsal root and sympathetic ganglia, and in the nerves and sensory corpuscles supplying digital skin. The Anatomical Record. 1994;240:579-588

[78] Stark B, Risling M, Carlstedt T. Distribution of the neurotrophin receptors $\mathrm{p} 75$ and trkB in peripheral 
The Glial Cell of Human Cutaneous Sensory Corpuscles: Origin, Characterization... DOI: http://dx.doi.org/10.5772/intechopen.91815

mechanoreceptors; observations on changes after injury. Experimental Brain Research. 2001;136:101-107

[79] Calavia MG, Feito J, LópezIglesias L, de Carlos F, García-Suárez O, Pérez-Piñera $P$, et al. The lamellar cells in human Meissner corpuscles express TrkB. Neuroscience Letters. 2010;468:106-109

[80] Pawson L, Pack AK, Bolanowski SJ. Possible glutaminergic interaction between the capsule and neurite of Pacinian corpuscles. Somatosensory \& Motor Research. 2007;24:85-95

[81] Pawson L, Prestia LT, Mahoney GK, Güçlü B, Cox PJ, Pack AK. GABAergic/ glutamatergic-glial/neuronal interaction contributes to rapid adaptation in pacinian corpuscles. The Journal of Neuroscience. 2009;29:2695-2705

[82] Tsunozaki M, Bautista DM. Mammalian somatosensory mechanotransduction. Current Opinion in Neurobiology. 2009;19:362-369

[83] Lumpkin EA, Marshall KL, Nelson AM. The cell biology of touch. The Journal of Cell Biology. 2010;191:237-248

[84] Gu Y, Gu C. Physiological and pathological functions of mechanosensitive ion channels. Molecular Neurobiology. 2014;50:339-347

[85] Paluch EK, Nelson CM, Biais N, Fabry B, Moeller J, Pruitt BL, et al. Mechanotransduction: Use the force(s). BMC Biology. 2015;13:47

[86] Ranade SS, Syeda R, Patapoutian A. Mechanically activated ion channels. Neuron. 2015;87:1162-1179

[87] Roudaut Y, Lonigro A, Coste B, Hao J, Delmas P, Crest M. Touch sense: Functional organization and molecular determinants of mechanosensitive receptors. Channels (Austin). 2012;6:234-245

[88] Fleming MS, Luo W. The anatomy, function, and development of mammalian $A \beta$ low-threshold mechanoreceptors. Frontiers in Biology (Beijing). 2013;8(4). DOI: 10.1007/ s11515-013-1271-1

[89] Montaño JA, Calavia MG, GarcíaSuárez O, Suarez-Quintanilla JA, Gálvez A, Pérez-Piñera $P$, et al. The expresssssion of $\mathrm{ENa}(+) \mathrm{C}$ and ASIC2 proteins in Pacinian corpuscles is differently regulated by TrkB and its ligands BDNF and NT-4. Neuroscience Letters. 2009;463:114-118

[90] Calavia MG, Montaño JA, García-Suárez O, Feito J, Guervós MA, Germanà A, et al. Differential localization of acidsensing ion channels 1 and 2 in human cutaneus pacinian corpuscles. Cellular and Molecular Neurobiology. 2010;30:841-848

[91] Cabo R, Alonso P, Viña E, Vázquez G, Gago A, Feito J, et al. ASIC2 is present in human mechanosensory neurons of the dorsal root ganglia and in mechanoreceptors of the glabrous skin. Histochemistry and Cell Biology. 2015;143:267-276

[92] Alonso-González P, Cabo R, San José I, Gago A, Suazo IC, García-Suárez O, et al. Human digital Meissner corpuscles display immunoreactivity for the multifunctional ion channels Trpc6 and Trpv4. Anatomical Record (Hoboken, N.J.). 2016. 2016;300:1022-1031

[93] Pawson L, Bolanowski SJ. Voltagegated sodium channels are present on both the neural and capsular structures of Pacinian corpuscles. Somatosensory \& Motor Research. 2002;19:231-237 



\title{
Chapter 3
}

\section{Vestibular System: Anatomy, Physiology, and Clinical Evaluation}

\author{
Dmytro Illich Zabolotnyi and Nina Serhiivna Mishchanchuk
}

\begin{abstract}
Abstract Studies on vestibular system have brought new experimental studies, clinical examinations, and the development of effective treatment for a number of diseases of this system. In particular, vestibular paroxysmal positional disorders of peripheral and central origin have been studied. The main criteria for differential diagnosis of these disorders have been determined. Vestibular dysfunction in canalolithiasis and cupololithiasis has been investigated clinically and histologically. Effective therapeutic and prophylactic positional maneuvers of three types have been introduced into clinical practice. They were developed taking into account the anatomical and physiological features of the vestibular system. Currently only $20 \%$ of vestibular reactions, in particular, using electronystagmography test (ENG), are estimated in the horizontal plane. Videonystagmography (VNG) gives the possibility of video recording of nystagmus in the directions of semicircular channels (vertical, diagonal, horizontal). The vestibular evoked myogenic potential test (VEMP) is being widely used in clinical practice. Magnetic coils and scanning laser ophthalmoscopes are gaining increasing significance in examining patients. A brief information on vestibular disorders after the Chornobyl nuclear power plant accident is also given.
\end{abstract}

Keywords: vestibular dysfunction, vertigo, postural balance and control, spontaneous, positional, and experimental nystagmus

\section{Introduction}

Sensory functions, in particular, vestibular and auditory ones, represent a reliable criterion for assessing human health, social adequacy, professional suitability, and working capacity.

Symmetry of the anatomical-topographical localization of these systems and proper space orientation provided by them are important regarding all social life aspects. The vestibular system plays a crucial role in space orientation, both at rest and in movement, as well as in professional activity of the person. Its effect onto the motor memory mechanisms, underlying all human motor activity forms, including the professional ones, is extremely important. The vestibular system, with its extremely keen sensitivity, dynamism, and high informativeness of symptoms of the ear labyrinth diseases, as well as different parts of the brain, significantly exceeds the informativeness of all other analyzers. It is the first system to respond to any disorders in the body. 
Auditory system provides for social communication function. Binaural hearing impairment leads to spatial orientation disorders. Vestibular and auditory sensory systems stimulate cortical processes, being responsible for various activities, postural balance and control, emotions, and creative thought. Therefore, the need for a comprehensive clinical study of vestibular and auditory disorders in order to increase the effectiveness of prevention and treatment, correction and rehabilitation, identification of issues of professional suitability, and work capacity is very important.

\section{Overview and anatomy of the vestibular system}

Vestibular system (VS) is the most mysterious biological system. It was the first to develop during the embryogenesis. The vestibular system consists of the peripheral and central components, which have a complex structure that has not been fully understood. Its peripheral components are located in the paired ear labyrinth of the pyramid of the temporal bone. Its central components are composed of the conductive part, vestibular nuclei in the brain stem and cortical representation [1,2].

Bony labyrinth is located in the temporal bone. The membranous labyrinth is located in the middle of the bony labyrinth, resembling the shape of its small cavities. Membranous labyrinth is fixed to the inner wall of the bony labyrinth by means of helical formations acting as shock absorbers. Due to this, it is held in relation to the bony labyrinth, being suspended. Membranous labyrinth consists of the peripheral acoustic section of the cochlea, where the auditory receptor organ of Corti is located, as well as the peripheral component of the vestibular system. The peripheral component of the vestibular system is represented by the vestibulum containing spherical (saccule) and elliptical (utricle) sacs that form the receptor otolith organ. Another three semicircular canals of the peripheral vestibular system are located in the membranous labyrinth [3].

Each canal is thickened and enlarged at one side, resembling ampoules by shape (ampullary receptor organ), and it has a smooth terminal region that connects to the vestibulum on the other side. The three semicircular canals are located approximately at straight angle to each other. Therefore, they may sense angular motion, conducted in any plane or direction. One canal is horizontal and the other two (front and back) are vertical. The perception of head movement is provided by activation of the right and left semicircular canals, which are located within the movement plane, thus forming functional pairs.

The membranous labyrinth is washed by perilymph fluid, which in chemical composition is similar to the cerebrospinal fluid. The middle of membranous labyrinth contains endolymph. It is intracellular fluid that provides the helix, otolith, and ampullar apparatus with oxygen, nutrients, and hormones. The potassium concentration in it is high $(144 \mathrm{mmol} / \mathrm{L})$, and the concentration of sodium is low $(5 \mathrm{mmol} / \mathrm{L})$. The mechanisms of endolymph formation and its circulation in the membranous labyrinth have not been yet well understood. The membranous labyrinth is elastic, and its size can increase by $2-3$ times, filling all the "cavities" of the bony labyrinth, for example, in the case of ascites and other pathological conditions [4].

In the receptor otolithic apparatus, spherical (saccule) and elliptic (utricle) sacs contain hairy receptor cells covered by supporting dense layers of cells called overhead covers. These receptor cells are sensitive to linear acceleration. They flex in response to appropriate irritation. The clusters of the neuroepithelial receptor cells of the otolith apparatus, which are located between the supporting cells in elliptical and spherical sacs, interweave, forming loops. Calcium salt microcrystals are located in these loops; they are called otoliths [5]. 
The receptor structures of the ampullar apparatus, three semicircular canals (anterior, posterior, and horizontal), are located in mutually perpendicular planes. The smooth terminal parts of two semicircular canals (anterior and posterior) are joined into a single canal that enters the utricle. It is connected to the receptive auditory structures of the Corti organ. Endolymph, which is produced by the vascular strip of the helix, passes through this canal into the vestibular endolymphatic canals. This important fact, as the authors emphasize [4], is significant for various ear diseases and pathological changes of the temporal bone, as well as the brain, accompanied by vestibular disorders.

The hair cells of the vestibulum and the ampoules of three semicircular canals generate a receptor potential, which through the synapses (by production of acetylcholine) transmits signals to the terminal regions of the vestibular nerve fibers.

The vestibular nerve fibers originate in the vestibular ganglion. It is located deep in the internal auditory canal. This node consists of bipolar cells. The dendrites of these cells penetrate into the bony labyrinth through the internal auditory canal opening, approaching the saccule and utricle receptor structures, as well as the ampullar semicircular canal receptor structures. Axons of these cells, joining the helix nerve, represent the vestibulocochlear (auditory vestibular) nerve-the VIII cranial nerve. In the internal auditory canal, this nerve meets facial nerve-the VII cranial nerve-proceeding into the cavity of the skull; then in the thickness of the rhombic fossa of the medulla oblongata, four-paired vestibular nuclei enter: upper and lower and lateral and medial [6].

Reflex pathways originate in the vestibular nuclei, and these pathways are in various ways related to different systems and organs in the body. There are five pathways:

1. Tractus vestibulo-spinalis, through which impulses from the vestibular nuclei reach the voluntary muscles of the spinal cord anterior horns.

2. Tractus vestibulo-longitudinalis joins vestibular nuclei with nuclei of the III, IV, and VI cranial nerves, resulting in responses produced by the oculomotor nerves-varieties of nystagmus.

3. Tractus vestibulo-cerebellaris joins the nuclei with the cerebellum.

4. Tractus vestibulo-reticularis joins vestibular nuclei with the vagus nerve nuclei in the reticular formation, producing reflexes in the internal organ smooth muscles.

5. Tractus vestibulo-cortikalis joins the vestibular nuclei with the temporal lobes of the cerebral cortex through multisynaptic connections, with the midbrain crossway.

It should be noted that disorders of the vestibular system occur at any of its levels from the receptor structures of the ear maze to the cortical representation. When leaving the internal auditory canal, V, VI, and VII pairs of cranial nerves are located near the VIII pair of cranial nerve as well as a group of pairs of caudal (IX-XII) cranial nerves. They reflect the lesions of structures located near the vestibular system.

It should be emphasized that in the cases of disorders in the area of the labyrinth, temporal bone, and cerebellar angle, vestibular disorders are often accompanied by unilateral auditory disorders. Central vestibular disorders are less 
often combined with auditory disorders. In the brain stem, four pairs of vestibular nuclei are located in a relatively small anatomical space. The presence of these nuclei indicates the great importance of the vestibular system in evolutionary terms $[2,3,5]$.

Vestibular disorders very often occur in patients with brainstem pathology. The nuclei of the III, IV, and VI cranial nerves are located with the vestibular conduction pathways, the damage of which is accompanied by diplopia. Pathology of the $\mathrm{V}$ cranial nerve is manifested by a decrease in the sensitivity of the skin of the face. Facial (VII) nerve disorders lead to facial distortion, speech disorders, pathology of the IX-X cranial nerves, and disorders of swallowing and articulation. Vestibular disorders through close anatomical connection with the cerebellum can contribute to the development of cerebellar ataxia [4].

\section{Physiology of the vestibular system}

Researchers have been studying functions of the ear labyrinth for centuries. Describing the feelings during self-spinning, the symptoms of responses occurring in this case were reported: vertigo, balance problems, sweating, nausea, vomiting, changes in heart rate and blood pressure, and respiratory disorders. In the study on birds, destroying semicircular canal of the ear labyrinth, it was first found that sensory perception is based on stimulation of ampullary receptors. In further experiments it was reported that semicircular canals are receptor organs that perceive rotational motions. These movements cause shifting of the endolymph, which causes irritation of receptor neuroepithelial cells in the semicircular canal ampoules, producing nerve impulse [6].

Purkyně [7], reporting on his feelings in the rotation, first described the symptoms of the reactions that occur in this case: vertigo, imbalance, sweating, nausea, vomiting, changes in heart rate and blood pressure, and breathing.

Florens [8] in the experiment on birds, destroying the semicircular canals of the ear labyrinth, first established that the stimulation described by J. Purkiunje was based on the irritation of the ampullary receptors of these canals. These observations initiated the experimental study of the vestibular labyrinth. It was defined that semicircular canals are receptive organs that perceive rotational movements. These movements cause displacement of the endolymph, leading to irritation of receptor neuroepithelial cells in ampoules of semicircular canals, resulting in a nerve impulse.

Evald [9] conducted unique experimental studies and discovered the patterns of functioning of vestibular semicircular canals, which are known as the three laws of Evald:

1. Nystagmus always occurs in the plane of the semicircular canal irritation.

2. Ampullopetal (toward the canal ampulla) movement of the endolymph causes richer response than the ampullofugal (toward the smooth terminal part of the canal) one.

3. Nystagmus is always directed toward more active semicircular canal.

4. Prominent scientists of the last century presented clinical and experimental evidence of the vestibular system connection with other body systems and organs, the dependence of response on both strength of the stimulus and functional state of the CNS (cited by [6]). 
If vestibular system is affected, three types of pathological responses may occur: vestibular sensory, vestibular somatic, and vestibular vegetative, with different levels of manifestation.

I. The vestibulo-sensory subjective pathological responses are caused by the processes that occur in the cerebral cortex. These structures analyze and synthesize obtained stimuli. The disorder here may be manifested as a pathological subjective sensation of continuous or periodic vertigo of different direction and intensity, as well as orientation disorders with the reflex action. Vertigo is the most widespread specific sensation after pain, which indicates a disease. The vestibulo-sensory subjective pathological responses originate in the vestibulo-cortical tract.

II. The vestibulo-somatic objective pathological responses are manifested as muscle reflexes, accompanied by objective spontaneous static and kinetic equilibrium disturbance, as well as pathological objective vestibulo-oculomotor reflexes: spontaneous or positional nystagmus of various direction and intensity (horizontal, rotating, vertical, diagonal, multiple). They are an objective feature of the vestibulo-sensory subjective sensation vertigo of appropriate orientation. These reflexes are provided via tractus vestibulo-spinalis, tractus vestibulo-longitudinalis, and tractus vestibulo-cerebellaris.

III. The vestibulo-vegetative objective pathological responses arise in the receptor vestibular apparatus, as a response onto the smooth muscle of the internal organs' irritation (overpronounced response, hyperreflexia; suppressed response, hyporeflexia) with symmetric or asymmetric sensitivity due to vestibular pathologies, as well as intense and prolonged vestibular loads. These responses occur via tractus vestibulo-reticularis. They often lead to, combined with other vestibular responses, the development of various symptom complexes, including various forms of kinetosis, formerly called motion sickness (marine, air) [6, 10, 11].

These responses are differently significant for human life. As for professional activity, the vestibular vegetative and vestibular sensory responses are the most important. The first ones cause vegetative discomfort, in which a person feels bad and his/her workability is reduced. Others disorient a person in space and can cause serious danger when performing work that involves precise and coordinated movements.

According to the literature [12], up to $20 \%$ of the professionals whose jobs are related to the effect of prolonged or elevated vestibular system stimuli may become disabled due to vestibular disorders. Such professionals, as a result of movement discoordination or space disorientation, can make professional mistakes, which may cause man-made accidents or even catastrophes.

In anatomical, physiological, and functional relationship between the vestibular and auditory systems, the relation between the nuclear areas and different central nervous system formations and cerebral circulation contributed to the development of otoneurology. Progress in otosurgery and neurosurgery has identified a separate medical specialty: otoneurosurgery.

Pressure changes and various pathological changes associated with purulent and non-purulent processes in the tympanic cavity cause impaired middle-ear sounding function. These changes reach the window of the helix and vestibulum, causing sensory and neural hearing loss and vestibular dysfunction.

The structural, vascular, toxic, allergic disorders of the labyrinth may lead to hyperfunction of the endolymph and various vestibulopathies and labyrinthopathies.

Vestibular system, as a system consisting of paired organs, functions by the lever principle. The receptor hairy cells in each of the semicircular canals are located so that shift of the cupula into one side increases afferent input along the vestibular nerve directed toward that side and decreases toward the opposite side. 
For example, turning the head to the right will increase afferent input of the right lateral semicircular canal and decrease afferent input of the left one. As a result, two conclusions can be made, based on the above mentioned: (1) vertigo may develop at unilateral hypofunction state, even at rest; (2) in the case of complete dysfunction of the semicircular canal on one side, the functioning semicircular canal on the opposite side may perceive head movements in both directions of appropriate plane (by decreased or increased afferent input perception). This ability of the preserved labyrinth to perceive head movements represents the basis of vestibular compensation, providing for restored vestibular functions with unilateral vestibular dysfunction (VD) [12].

The adequate stimulus of the three semicircular canal ampulla apparatus receptor structures is acceleration and slowing during rotation movements. The receptor cells may shift due to the endolymph inertia shift. At the movement acceleration stage, it will be the maximum one. While rotating the head with steady speed, the endolymph shift gradually decreases, and in 15-20 s of rotation it attains the background properties. If to stop movement, the cupula shift turns the cupula toward the opposite side. This phenomenon represents the "stop-stimulus" while conducting classical rotation test using Barany rotational chair. If to compare duration of the caloric test, or the bilateral caloric cold and heat stimulation as well as the post-rotation nystagmus after rotating to the left and right, we should assess the symmetry or asymmetry of vestibular functions [1].

An adequate stimulus of the otolith organ results in acceleration or slowing down at the straight-line movements. As the gravity force is a linear acceleration, the otolith receptors perceive head inclination movements regarding the earth gravity force vector. Absent action of the gravity force onto the otolith organ at null gravity causes significant disturbance in the body, which are thoroughly studied by cosmic medicine. The sensitivity of otolith hairy cells to linear acceleration is determined by the mass of heavy calcium crystals-otoliths. Elliptic sacs of the otolith organ, situated close to horizontal plane, are more sensitive to acceleration within this plane. Spheric sacs are located in almost vertical plane, so they are more sensitive to acceleration and slowing down within a vertical plane. It is necessary to stress that the head movements are the combined ones, i.e., they are accompanied with linear and angular acceleration within various planes and directions. Due to joint functioning of four otolith organs and six semicircular canals, it is possible to perceive complex movements in space $[11,13,14]$.

The nystagmus subtypes are the most valuable specific and objective characteristics of acute peripheral, chronic, or acute progressing central VD. The nature of these nystagmuses has not been understood completely. It is supposed that the standard tone of the eye-moving muscles is supported by the impulses of similar symmetric force which derive from the both labyrinths. When we irritate the labyrinth, there appears reflex asymmetry from the eye-moving muscles, expressed as a gradual turning away of the eyes toward the muscle hypertonia side, with gradual nystagmus component developing. Gradual turning away of the eyes causes instant response from the central (cortex) parts, so the eyes turn to the previous position. This is how a quick component of the spontaneous or other nystagmus may appear. The confirmation of cortex genesis of quick nystagmus component is its absence in the case of unconsciousness or narcosis. The slow nystagmus component is preserved in these cases. The nystagmus direction is determined by its quick component [12].

There are three stages of spontaneous nystagmus: in the first phase, when the nystagmus is manifested when the eyes turn away toward the quick component side; in the second phase, when the eyes move toward the quick component or when a person looks straightforward; in the third phase, when the eyes look toward the quick component, straightforward and to the opposite side [13]. 
The studies established that the peripheral or labyrinth-borne spontaneous nystagmus, as a rule, by its intensity is a small- or medium-swinging; by its direction the nystagmus may be horizontal or horizontal-rotating and rhythmical, with distinctly changing quick and slow phases [12].

Peripheral nystagmus in the acute stage lasts from the first 2-3 hours to 2-3 days, being directed toward acute stimulation of the labyrinth receptor structures. With gradual labyrinth depression, the nystagmus changes its direction toward the healthy side. In the case of the VD acute stage of peripheral syndrome type, the spontaneous horizontal or horizontal-rotating nystagmus is accompanied with sensory subjective sensation, horizontal vertigo toward labyrinth hyperreflexia side and, in the case of depression, toward the opposite intact side. So, shifts in statics and movement coordination, keeping to the vector laws, are observed the same, directed toward stimulation side or toward the healthy side in depression.

The specific acute vestibular symptoms are accompanied by considerable general discomfort, manifested dually; its first manifestation is characterized by nausea, vomiting, and vascular disorders associated with sympatho-adrenergic crisis. Here we may observe increased arterial pressure, tachycardia, hyperemia, and skin dryness, as well as dry oral mucosa. Its second manifestation is characterized by decreased arterial pressure, bradycardia, pale skin and mucosa, extreme sweating, and salivation $[13,14]$.

The acute vestibular syndrome requires urgent medical aid. As the authors observe [14-16], the peripheral vestibular syndrome is characterized by high capacity for compensating the affected functions due to central regulating mechanisms. In 2-4 weeks the spontaneous nystagmus decreases in intensity and then disappears completely. At the same time, vertigo decreases; kinetic and then static equilibrium functions are restored.

The pressure changes and pathological disorders in purulent and non-purulent processes in the external ear and tympanic membranes cause problems with the sound-conducting function of the middle ear. These pathological changes through the helix window and vestibule may migrate onto the ear labyrinth. In the labyrinth, under the effect of these conditions, the content and shift of endolymph movement may be changed. These endolymph changes lead to the VS receptor structure impairment, and finally they result in sensorineural deafness and vestibular dysfunction.

In the case of the acute central vestibular syndrome (pathology of the cerebellopontine angle or the pons bodies), there may be other characteristics of spontaneous nystagmus observed. It may be large-swinging or medium-swinging. It may develop within various planes: the vertical, diagonal, horizontal, or multiple. In some cases, spontaneous nystagmus is defined as a tonic-clonic or tonic (with prolonged average angular speed of the slow stage of nystagmus or decrease of its frequency). Spontaneous nystagmus in the case of the central vestibular syndrome is continuous and may progress in intensity [12].

\section{Clinical disorders of the vestibular system}

\subsection{Benign paroxysmal positional vertigo (BPPV)}

Benign paroxysmal positional vertigo (BPPV) is the most frequent cause of light-headedness. Its treatment has become possible due to application of the original correction methods. The main sign of the BPPV is short-lasting vertigo attacks, which appear when a person abruptly changes his head position related to the gravity vector, for example, changing from the vertical onto the horizontal position, when a 
person lies down, or vice versa, when he gets up, or upturns in the bed, or abruptly throws his head back. These attacks of vertigo more often appear in the morning after sleep. They are mostly manifested when the person first changes body position after sleep. They are weakened with repeated head movements. The patients' complaints are very characteristics, quite often basing on the complaints it is possible to diagnose the condition and define the affected side. The patient quite often shows himself, from what side vertigo appears $[1,4,10,12]$.

If the vertigo and nystagmus are horizontally or horizontally rotating directed, this shows us that the horizontal (lateral) semicircular canal is affected. The provoking moment may be when a patient inclines his head toward the affected ear side. The vertigo may be accompanied with nausea and vomiting. The attack appears after a person moves his head, in 1-2 s and lasts for 30-60 s.Vertigo and nystagmus depend on the head movement. If a person looks upward, vertigo and nystagmus are vertical. Vertical nystagmus in this case evidences about excitement of receptors of the posterior semicircular canal. BPPV may develop at any age, most often from 50 to 70 years, affecting women twice as often as men. It may develop after prolonged bed stay, particularly after craniocerebral traumas $[11,14,16,17]$.

The diagnosis is established due to position tests. The position tests should be conducted with all patients complaining of the vertigo even if a patient's complaint is typical for the other vestibular disorders (Meniere's disease, vestibular neuronitis, migraine, and various etiology labyrinth diseases). When there are no effective method of the primary disease treatment, it is always possible to relieve the vertigo, related to the BPPV $[12,15]$.

According to the cupulolothiasis theory, which was histologically confirmed, in the ampulla of the posterior canal, which is located lower than the other canals, otoliths may deposit more frequently. They, due to various reasons, leave the otolith membrane and stick to the cupula walls. The cupula walls with otoliths become heavy. They become a sensor of linear acceleration instead of the sensor of the angular one though this theory cannot explain many peculiarities of nystagmus [4].

The canalolithiasis theory explains the positional nystagmus signs. Otoliths, according to this theory, do not stick but freely float in the endolymph. As they narrow or obstruct the canal diameter, they cause positional vertigo. As they travel along the canal, otoliths disrupt free shift of the endolymph from the utricle or to the utricle. This theory allows predicting the direction, latent period, and duration of the nystagmus, as well as the changes of its characteristics at various positional maneuvers [4].

In 10-20\% of patients suffering from BPPV, lateral semicircular canals have been affected. There are two types of them: related to canalolithiasis (more widespread) and a rarer one, cupulolithiasis. Identification of the lateral semicircular canal BPPV is characterized by some features which were earlier considered to be characteristics of the central positional vertigo. In the case of the lateral semicircular canal canalolithiasis, the patient experiences vertigo when he turns his head to sides, being supine. Changing the position from the supine onto the sitting one and vice versa is not accompanied with significant symptoms. Exacerbations with the lateral canal BPPV are less prolonged than those with the posterior canal affected. The cause of lateral canal BPPV as a canalithiasis type is the invasion of aggressive otoliths in this canal. Remissions are often observed in patients with lateral canal BPPV as otoliths can easily leave the lateral canal during head movements. On the contrary, otoliths do not move from the posterior canal independently due to the anatomical position (it is located below all other canals). A quick result from the maneuvers testifies to the lateral canal BPPV. BPPV anterior canal is used very rarely $[4,6,17]$.

The Dix-Hallpike test is the technique used to diagnose posterior semicircular canal BPPV. A patient is brought from sitting to a supine position, with the head 
turned $45^{\circ}$ to one side and extended over the end of the stretcher. In the case of left posterior semicircular canal dysfunction, the test will first cause an increased and then decreased nystagmus that occurs after a short latent period. After returning to the starting position, the nystagmus will change the direction [15-17].

A number of positional maneuvers have been developed to treat BPPV. They are aimed at removing conglomerates of otoliths from semicircular canals. At present, maneuvers developed by Brandt and Daroff and Semont and Epley maneuvers are used. Within the theory of canalolithiasis, Brandt and Daroff were the first to come up with a set of exercises, including head movements that destroy the otolith conglomerates. Their fragments move to the other sections of the labyrinth, where they may be stationary. Therefore, they do not affect the function of semicircular canals [4].

These maneuvers, when applied correctly, are always effective but $50 \%$ relapse. In case of relapses, it is recommended to repeat the maneuver that was more effective at the first attack $[12,14]$.

\subsection{Vestibular neuronitis}

Vestibular neuronitis is a common cause after BPPV peripheral vestibular disease. Clinically, it is manifested by vertigo in the direction of a healthy ear, by spontaneous nystagmus of a horizontally rotary direction to a healthy ear. Functional tests check various degrees of hyporeflexia to the affected ear and postural abnormalities toward a healthy ear. It is considered that vestibular neuronitis is of viral etiology. It is recommended to prescribe glucocorticoids at first as they effectively reduce functional disorders and have a strong anti-inflammatory effect. In addition, they accelerate the central mechanisms of vestibular compensation for postural disorders. Antiviral drugs are less effective [10].

\subsection{Meniere’s disease}

Meniere's disease refers to the peripheral diseases of both vestibular and auditory systems. During an acute attack in the excitation phase in the affected labyrinth, there occurs a strong vertigo of the horizontal direction with horizontal nystagmus, postural disorders, and significant vestibulo-vegetative pathological reactions. At the same time, there is a strong noise in the affected ear, hearing loss, feeling of pawning and fullness in it, and the phenomena of sound discomfort on enhanced sound stimuli. In the phase of inhibition of the labyrinth, vertigo and nystagmus change the direction toward a healthy labyrinth. Postural disorders gradually settle, and vestibulo-vegetative pathological reactions disappear. Within functional stimulation, hyporeflection of the nystagmus is fixed from the side of the affected labyrinth. At the same time, noise, stuffiness, and fullness in the ear decrease, and the effects of sound discomfort are reduced. These facts indicate a common mechanism of the processes of excitation and inhibition in both sensory systems in this disease $[6,10,18,19]$.

It was histologically confirmed that the basis for Meniere's disease is the periodic accumulation of endolymphatic dropsy (endolymphatic hydrops). An acute attack of vestibular dysfunction lasts from 30 minutes to 3-4 hours and recurs at different intervals. One of the effective treatment methods for Meniere's disease is shunting of a damaged labyrinth, vestibular gymnastics, and salt restriction. Long-term betahistine treatment is often prescribed to prevent attacks and reduce their manifestation. However, the causes of its occurrence have not been fully understood $[11,12,18]$. 


\subsection{Migrainous vertigo}

Migrainous vertigo is the most common form of spontaneous recurrent systemic vertigo. It occurs at any age, from preschoolers to older adults. Family history is often noted, indicating the contribution of genetic factors. It occurs with an aura less often than without an aura. During the attack, transient symptoms occur, such as atrial fibrillation, unilateral paresthesia, and aphasia. These symptoms are often preceded by headaches. Migraine-associated vertigo increases when the position of the head changes. The duration of symptomatic episodes when positional nystagmus can be detected may take several days. Relapses occur more frequently than BPPV. Migrainous vertigo is referred to as central positional vertigo [2, 3, 5, 19].

\subsection{Central positional vertigo}

Central positional vertigo occurs not frequently, in less than $5 \%$ of patients with vertigo. But it is a sign of a serious disease that is associated with structural damage of central divisions of the vestibular system and lobes of the cerebellum. Therefore, central lesion should be excluded first of all in patients with positional vertigo. The most reliable differential feature of BPPV and central positional vertigo is the direction of positional nystagmus. In the case of BPPV, by stimulation in the plane of a certain canal, positional nystagmus characteristic for this canal is typical. When stimulating horizontal (lateral) canalis semicircularis, horizontal positional nystagmus always occurs. The direction of the central positional nystagmus is often not correlated with the stimulating canal. Pure vertical or rotary nystagmus is a sign of central vestibular disorder. In patients with central vertigo, in the absence of changes in MRI, it is possible to suspect a migrainous or other vertigo $[14,16,19]$.

\subsection{The effect of Chornobyl nuclear power plant accident on vestibular system}

Our own experience $[20,21]$ of vestibular examinations for more than three decades of 8136 participants of Chornobyl nuclear power plant accident indicates that the patients had vestibular and sensorineural dysfunction. These two dysfunctions are characterized by a predominant disorder in the central (brain stem and subcortical) structures. The priority of changes (preclinical and early clinical) in the central departments of the vestibular and auditory systems, the dependence of the degree of manifestation on the dose, and duration of radiation exposure indicate the parallelism of the common mechanism under the influence of the radiation factor.

The occurrence of deterministic radiation effects of these dysfunctions by the type of central syndrome in radiation doses greater than $0.20 \mathrm{~Gy}$ has been investigated. Mathematical modeling determined the dependence of these effects on the age, magnitude of the dose, and duration of stay in the exclusion zone of the Chornobyl power plant, both by clinical electrophysiological and audiometric psychoacoustic and electroacoustic methods. Elongation of magnitudes of the latent periods of the components $\mathrm{P}-2$ and N-2 of auditory evoked long latency or cortical potentials (AEP) in patients with progressive sensorineural hearing loss (SHL) is characterized by slowing the intelligibility of speech and its paradoxical decrease in the conditions of high loads of noise. These features alongside with presbyacuzis praecox indicate common inhibitory processes of subcortical and cortical structures of the auditory system [20,21].

At the same time, there was a decrease in the parameters of the induced nystagmus reaction and a decrease in the postural balance, as well as changes in the manifestation of pathological vestibulo-vegetative reflexes inpatients with VD in 
late post-accident period. It has been proven on the basis of epidemiological studies that the inhibition processes in these systems can be regarded as a symptomocomplex of premature aging, with the development of cognitive, emotional, and volitional responses. They require regular treatment and prevention and rehabilitation measures to improve quality and life expectancy [21].

\section{Clinical tests for the vestibular system}

Determination of vestibular dysfunction of peripheral, central, or combined (mixed) character requires timely vestibulometric examination for differential diagnosis. This is necessary for the clinical needs of ENT specialists in the treatment of ear diseases (non-purulent and purulent otitis media, otosclerosis, sensory and neural deafness of different genesis, Meniere's disease, neoplasms of the ear).

Varieties of vestibular and acoustic dysfunctions may be detected by the physicians of other specialties to provide optimal medical care for neoplasms and traumas of the skull (including the temporal bone, brain), cardiovascular, neuropsychic, infectious, noise-vibrating, radiation-induced, endocrine, toxic, and other diseases [12, 14].

According to the clinical data [13,15-17], vestibular test methods should be economical, informative, and adequate to the patient's condition and the task of diagnostics and include the study of the functional state of other cranial nerves which may be required to provide a qualified advisory opinion. ENT examination, as well as voice and speech audiometry, is mandatory for vestibular examination.

In the case of unilateral or asymmetric bilateral disorder of sound-conducting or sound processing functions, or their combined disorder, it is necessary to carry out one of the following procedures: pure tone audiometry, impedancemetry, otoacoustic emission, registration of short-latent (brain stem) and long-latent (cortex) auditory evoked potentials. The results of the state of auditory function permit making certain focuses during vestibular examination and determining directions for further study of a sick person.

Schemes of vestibular surveys which include vestibular card surveys and a certain sequence of procedures were developed [6]. Scheme of vestibular examination includes:

1. The study of complaints, medical history, and past diseases using a standardized card or survey.

2. Investigation of spontaneous vestibular reactions (equilibrium functions, varieties of spontaneous, positional and pressor nystagmuses) and their registration.

3. Conducting vestibular experimental loads (functional stimulations) and their registration.

4. Analysis and evaluation of vestibular test results, making conclusions about the status of vestibular function and recommendations.

Special vestibular test cards or surveys include a list of general and specific complaints. The sensory subjective reaction-vertigo (the nature of the rotation of objects or their floating or self-rotation, the sense of failing, etc.) - is explained in detail. The direction of vertigo (left, right, up, down, chaotic) and postural disorders (while walking, in the darkness, in fatigue, during transport loads) should 
be determined as well as the presence of autonomic disorders (nausea, vomiting, changes in heart rhythm, changes in skin color, sweating). In cases of complaints about vertigo and postural disorders, attention is focused on the possibility of detecting specific objective spontaneous symptoms-registration of types of nystagmus and posturographic control of these complaints. Some patients pay attention to the fact that they have impaired vision fixation in vertigo. Visual performance is reduced, and fatigue occurs when using a personal computer or mobile phone. In the inter-onset, when there is no vertigo, vision fixation and visual acuity are restored.

The history of the disease is collected in detail: the sequence of its development and the combination with signs of disorders in other systems and organs (cardiovascular, neuro-psychiatric, endocrine, digestive, and musculoskeletal) and past diseases. Attention is paid to the presence of noise, its nature, the acuity, and asymmetry of hearing. Also the asymmetry of the pair of cranial nerves of V, VII, IX, and XII must be examined. Allergic or toxic manifestations are recorded during the survey.

In the course of clinical vestibular test, a large number of trials and tests have been accumulated for postural control. Some of them are of historical importance, and some are still in use, particularly, walking in a straight line, flank walking by steps to the right and left-for differential diagnosis of vestibular and cerebellar disorders.

Romberg's test (classical and sensitized) is the basis for determining the static and kinetic equilibrium in modern types of postural test techniques (in particular, stabilography-a mobile platform with processing of the center of "gravity" and Fourier oscillation analysis.). The speed of movement of the center of gravity in three directions (back and forth, left-right, up-down) is calculated quickly and accurately by a computer. Deviations in the performance of these methods are taken into account when assessing the degree of manifestation of disturbances of the equilibrium function. Postural tests are widely used in the differential diagnosis of peripheral and central balance disorders, for postural control during rehabilitation measures, for postural balance before and after cochlear implantation, for expert and prognostic purposes.

The next step in vestibular examination is the detection of spontaneous, positional, and pressor nystagmuses and registration of their characteristics [13,14]. At first, nystagmuses can be evaluated visually as well as using Frenzel glasses (+10-12 diopters, which have the backlight inside). A type of spontaneous nystagmus is a positional nystagmus that occurs in one of the head positions and can change its parameters depending on its change. The positional nystagmus is of peripheral or central origin.

Applying pressure, fistula test is of great importance in vestibular field in cases when fistula, prefistula, or dyskinesia in the bony labyrinth is suspected. As a rule, its presence is associated with the destruction of the capsule of bony labyrinth in the purulent process in the middle ear and in the temporal bone. Pressor nystagmus occurs most frequently in the lateral semicircular canal but can often occur in the anterior and posterior semicircular canals. In the presence of a fistula, the pressor nystagmus is directed in the direction of thickening of the air, and in the case of rarefaction (decompression) - in the opposite direction. Tullio phenomenon is characterized by under acoustic loads with vertigo and nystagmus. The Tullio phenomenon is often a barrier to the use of sound-enhancing equipment and hearing aids. The presence of a fistula in the dynamics of clinical observation and surgery is specified at the MSCT.

Barany tests [22] continue to be the most common methods of functional stimulation in clinical practice for the study of the characteristics of induced nystagmus. They are called classic caloric and rotating. The essence of the induced nystagmus reaction 
in caloric stimulation according to Barany is explained by the different movements of the endolymph during its cooling or heating of the liquid. In the caloric test, the patient bends his head back at $60^{\circ}$. In this location, the lateral and semicircular canals occupy a position when their ampoules are on top and the smooth end is below [22].

The caloric test is generally well tolerated. It can be performed in the case of the integrity of the eardrum, even in the state of unconsciousness, but in the absence of other obstacles. Temperature regimes can be changed depending on the tasks of the examinations and the accepted schemes in certain clinics.

Barany rotary test causes angular acceleration. In this test, the subject with the eyes closed and the head tilted forward $30^{\circ}$ rotates according to the program in a chair 10 times for $20 \mathrm{~s}$. in the lateral plane of the semicircular canals. These channels are of particular importance in people's life, because most frequently they make movements in the horizontal plane [22].

During the rotating test to the right, after stopping the chair the movement of the endolymph in the left semicircular duct will be ampullopetal, that is, the postlateral nystagmus reaction will be directed to the left according to Ewald's laws, and during the rotating test to the left, it will be directed to the right. The disadvantage of the probe is that each labyrinth cannot be inspected separately in isolation.

The Fitzgerald-Hallpike caloric test estimates the function of each of the lateral semicircular canals separately $[1,2]$. Before examination, the pathology of the tympanic membrane is excluded, and the head is raised up to $30^{\circ}$ forward, so that the lateral semicircular canals take a strictly vertical position, which will maximize the sensitivity of canals to thermal stimulation. Water at a temperature of 30 and $44^{\circ} \mathrm{C}$ is infused bilaterally (after 5-10 minutes) to the external auditory canal each time for $40 \mathrm{~s}$. Electronystagmography (ENG) records horizontal and vertical nystagmus, which occurs during cold calorization toward irritation and during hot calorization, in the opposite direction. A difference of more than $25 \%$ indicates an asymmetric lesion.

When infused with hot water $44^{\circ} \mathrm{C}$ in the same amount, the endolymph molecules rise up, causing the ampullopetal movement of the endolymph, which causes a caloric nystagmus reaction toward the irritated ear. The main advantage of caloric stimulation is monaurality, which allows surveying each labyrinth separately. This advantage is especially important for otoneurology. The disadvantages are limited possibilities for perforated ear drums, traumas, and neoplasms of the outer, middle ear, and ear labyrinth.

Electronystagmography (ENG) and videonystagmography (VNG) record varieties of spontaneous nystagmus and experimental nystagmus reaction to functional stimulation using special mass-produced instruments, with computer-aided analysis of their quantitative and qualitative characteristics $[1,6,10]$.

ENG is the first noninvasive quantitative and qualitative method of recording the difference of the corneo-retinal potential for the evaluation of spontaneous and induced nystagmuses in cold and warm caloric stimulation, as well as in rotational and optokinetic tests in the horizontal plane. ENG ensures accurate recording of results in patients with known vestibular disorders not only for clinical but for forensic and insurance medicine.

In caloric and rotational tests, the induced nystagmus reaction in the case of central vestibular syndrome is arrhythmic or dysrhythmic, with the presence of "silent fields," which indicates a violation of the central regulatory mechanisms of nystagmus (see dysrhythmic nystagmus in Figure 1).

At the same time, illusory sensory sensations - vertigo and autonomic reactions-arise, the degree of manifestation of which is taken into account when assessing the reactivity of the vestibular system. The more excitable it is, the greater and longer the illusory reactions will be. 
1)

2)

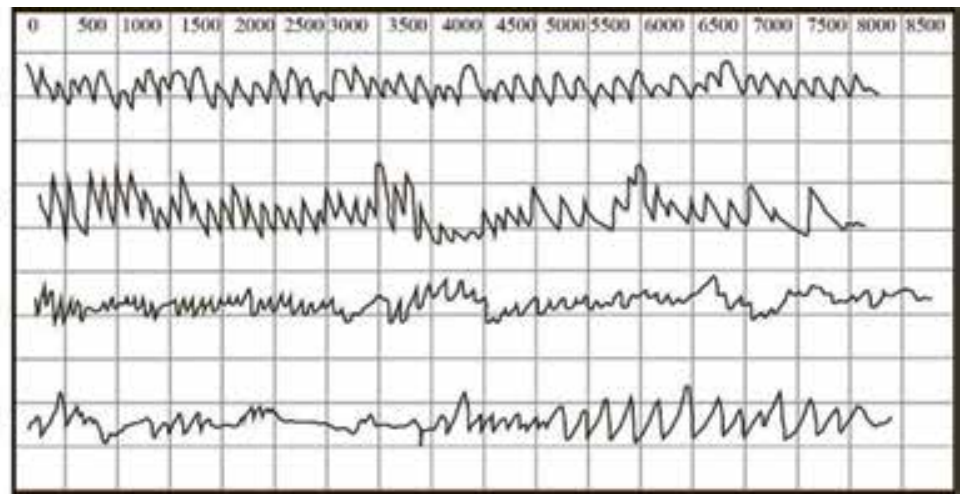

Figure 1.

ENG in caloric stimulation: (1) normoreflexia, (2) hyperreflexia, (3) hyporeflexia, and (4) dysrhythmia (obtained with permission from [6]).

Computer analysis of induced nystagmus responses to calorific and rotational stimulations can be performed every $10 \mathrm{~s}$ from the onset to attenuation, as also the duration of the induced and sensory responses can be determined and their changes tracked in the dynamics of the process and control of the treatment.

We would like to emphasize that caloric and rotational stimulations are based on the study of the functions of horizontal semicircular canal. It is considered that this is only an estimation of $20 \%$ of the functions of the vestibular system. However, the ability to capture the functions of horizontal semicircular canal is clinically important for otology and labyrinthology $[2,4]$.

The following variants of the state of the induced nystagmus reaction to functional loads are distinguished by ENG:

\section{Normoreflexia.}

2. Hyperreflexia, characterized by an increase in the qualitative and quantitative parameters of the induced nystagmus reaction on one side or on two sides. It indicates unilateral asymmetric or bilateral symmetric state of excitement of vestibular function; the symmetrical state of excitement of vestibular function is often registered at congenital and acquired kinetoses.

3. Hyporeflexia, which is characterized by a decrease in the qualitative and quantitative parameters of the induced nystagmus reaction on one side or on two sides. It indicates a one-way asymmetric or two-way symmetrical state of decrease in sensitivity of vestibular function. Symmetrical state of decrease in sensitivity in the absence of disturbances of the functions of equilibrium occurs in trained people of certain professions (pilots, athletes).

4. Areflexia is the complete absence of experimental nystagmus reaction on one side or two sides. It is confirmed by two stimuli-caloric and rotational.

5. Dissociated vestibular reactions are marked by dysrhythmia of experimental nystagmus with disharmony of all constituent reactions (vestibulo-sensory, vestibulo-vegetative, and vestibulo-somatic). This condition is observed in the central disorders of the vestibular function [6].

In addition to assessment of the condition of the vestibular function, the type of lesion, the degree of its manifestation, and stage of development will be determined. 
There are three types of vestibular dysfunction: (1) peripheral, (2) central, and (3) mixed.

There are three stages of manifestation of vestibular dysfunction and three stages of development-compensation, subcompensation, and decompensation $[6,11]$.

ENG has some limitations. It is important to emphasize that ENG records the function of the lateral semicircular canals and does not capture information about the anterior and posterior semicircular canals and spherical and elliptical sacs.

ENG testing is not sensitive to rotary, vertical, and other types of spontaneous and induced nystagmuses.

These examinations are now possible with the help of videonystagmography (VNG). VNG is the second noninvasive method which is increasingly used in clinical practice. On VNG, which is based on the principle of video telemetry of the eyeball movements, a video photo of spontaneous or positional nystagmuses is performed in different directions (vertical, horizontal, diagonal). It quickly and accurately captures horizontal and vertical eye movements, without artifacts (motion blinks or electrical noise when recording). VNG is performed with the eyes wide open. Eye movement is recorded with a camera recorder using a monocular and binocular mask. The resolution of the method is limited by the speed of the camera recorder shot (the most common currently available is the camcorder capacity of 100 frames per second).

Computer processing of video of pupil movements and light reflexes on the cornea are carried out, and two-dimensional image of eye movements is obtained. VNG registers Fitzgerald-Hallpike bithermal caloric stimulation with graphical image and digital computer analysis of its indices in relation to each other, which is important for clear determination of asymmetries in otoneurology (Figure 2).

Vestibular evoked myogenic potential (VEMP) testing has been studied in recent decades. In conducting this test, intense sound is used to stimulate one of the receptor otolith organs, the spherical sac. This stimulus elicits an electrophysiological response through the lower branch of the vestibular nerve.

The vestibulospinal reaction with relaxation in the ipsilateral sternocleidomastoid (SCM) muscle is measured by changing its activity. It is recorded using electromyography (EMG). SCM muscle is used because the response to this muscle is considered reliable due to the availability of EMG technology with electrodes on the skin surface. Initially, VEMP is recorded with a background muscle contraction and

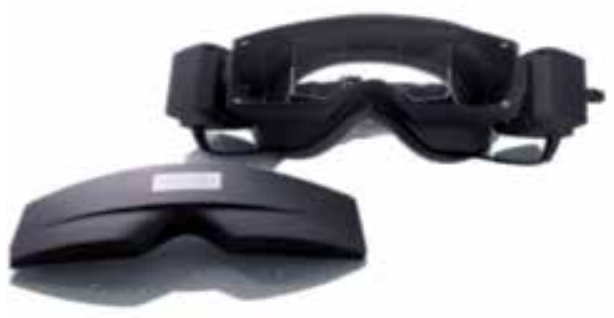

A)

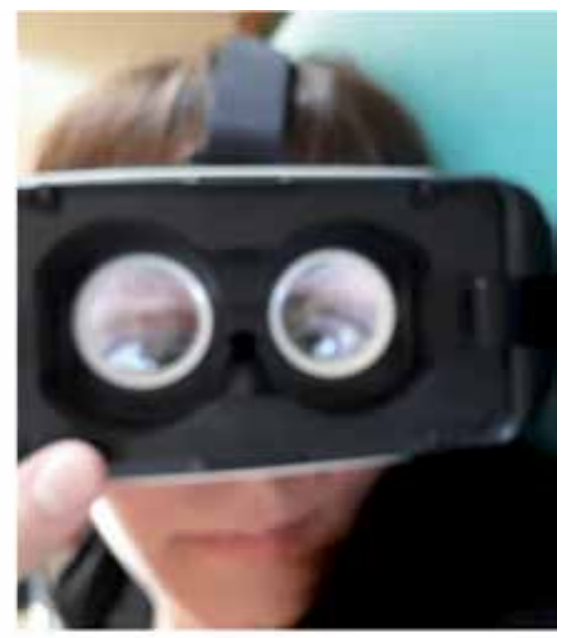

B)

Figure 2.

(A) Equipment for videonystagmography. (B) Examination of a patient with a videonystagmograph (from our own archive). 
then with a 90-100 dB acoustic click stimulation for 30 s from the ear to which the acoustic stimulus is applied. The VEMP test is considered promising for the assessment of labyrinth in norm and pathology [23].

Recently, methods of examination with the use of magnetic coils and scanning laser ophthalmoscopes have become increasingly important. The main purpose of these methods is differential diagnosis of peripheral and central vestibular and oculomotor disorders.

In patients with acute pathology of the external, middle, and inner ears of inflammatory nature and other genesis, functional loads at vestibulometry are performed with the implementation of rotational stimulation. Caloric stimulation is limited. With purulent chronic otitis media, complicated by cholesteatoma, retraction pockets, and other pathology, fistula (pressor) tests and rotational stimulation with mandatory appointment of MSCT with contrast are required.

In bilateral or asymmetric sensorineural hearing loss of different genesis, vestibulometric examination is performed using caloric and rotational stimulations, with consultations of psycho-neurologists, hematologists, vertebrologists, endocrinologists, and toxicologists.

The otoneurologic examination should necessarily include the studies which make it possible to detect structural changes in the state of the auditory and vestibular systems in the ear labyrinth, temporal bone, cervical spine, skull, and brain. They are performed using multispiral computed tomography (MSCT) and magnetic resonance imaging (MRI) with contrast.

Data on the functional status of sensory systems are needed for clinical physicians to obtain expanded information on diseases with consideration of morphological, etiological, and pathogenetic factors for nosological diagnosis.

To make conclusions the most commonly used classification of vestibular syndromes is used according to the syndromotopic scheme:

Peripheral and central syndromes are distinguished by this scheme. The peripheral syndrome includes labyrinthine and radicular ones. Central syndromes are divided into subtentorial and supratentorial. Subtentorial syndromes are subdivided into subnuclear, nuclear, and super-nuclear. Supratentorial syndromes include diencephalic-hypothalamic, subcortical, and cortical. Often combined vestibular syndromes are identified.

This classification is not universal. However, in the creation of a universal one, as emphasized by researchers [3-5], the obstacle is the "ubiquity" of the vestibular representation in the CNS. In addition, the subtle sensitivity to various changes in the internal and external environment and the globality of the vestibular symptom complex with similar symptoms, known as "vestibular triad," complicate the development of the principle of classifications of vestibular disorders according to the nosological principle.

Due to the anatomical, physiological, and functional closeness of the receptor structures of the auditory and vestibular systems, given the specificity of their disorders in the ear labyrinth, it is rational to isolate the peripheral cochleovestibular syndrome. Specific changes in the central departments of the vestibular and auditory systems are the bases for the formation of the central audio-vestibular syndrome and their combination—-for mixed audio-vestibular syndrome.

\section{Conclusions}

1. The presented anatomical and physiological bases of the vestibular system indicate the structural and functional interrelation of its peripheral and central departments in normal and pathological conditions. 
2. The parity and symmetry of the anatomical and physiological localizations of the vestibular and auditory systems play a crucial role in the spatial orientation in the state of motion and rest, in the formation of motor, sensory memory, and cortical stimulation of their activity.

3. The vestibular system is closely linked to the departments of the central nervous system, cerebral circulation, organs, and other systems of the body, so before others, it responds to the smallest changes in the internal and external environment.

4. The priority of changes in the central departments of the vestibular system under the influence of radiation has been proven.

5. It is established that the inhibitory processes in the vestibular and auditory systems as a result of radiation from the Chornobyl accident can be regarded as a symptomocomplex of premature aging of the organism with a decrease in cognitive and emotional-volitional reactions.

6. Along with the widespread use of objective electrophysiological methods of registration, in particular, electronystagmography, new diagnostic tests have been developed. The method of videonystagmography, scanning laser ophthalmoscopes, and magnetic coil examinations are gaining popularity. They contributed to the study of vestibular dysfunction in the positional paroxysmal vertigo of peripheral and central origins.

7. Three types of effective therapeutic and prophylactic positional provocative maneuvers have been implemented in clinical practice. They were developed by taking into account the anatomical and physiological features of vestibular system peripheral and central components.

Further experimental and clinical studies are needed to explore regarding the receptor functions of three semicircular canals and the otolith apparatus of the vestibular system. Developing new and refining existing objective methods for examining and recording varieties of spontaneous and experimental nystagmus not only in horizontal but also in other planes are necessary to enhance the assessment of vestibular functions in normal and pathological conditions.

The solutions to the important problems of vocational guidance; professional selection and career aptitude in sports, arts, maritime, aviation, and space technologies; and jobs related to ground and underground transportations are extremely needed. These professions are associated with heavy loads on the vestibular system. Particularly high demands are placed not only on vestibulo-vegetative and postural stability but also on vestibulo-sensory reactions. The development of preventive measures for safety and high endurance of the vestibular system, along with modern technological advancements, is of high priority. 


\section{Author details}

Dmytro Illich Zabolotnyi and Nina Serhiivna Mishchanchuk ${ }^{*}$ O.S. Kolomiychenko Institute of Otolaryngology, National Academy of Medical Sciences of Ukraine, Kyiv, Ukraine

*Address all correspondence to: nsmisch@i.ua; nsmishch@gmail.com

\section{IntechOpen}

(c) 2020 The Author(s). Licensee IntechOpen. This chapter is distributed under the terms of the Creative Commons Attribution License (http://creativecommons.org/licenses/ by/3.0), which permits unrestricted use, distribution, and reproduction in any medium, provided the original work is properly cited. $(\mathrm{cc}) \mathrm{BY}$ 


\section{References}

[1] Gulya AJ, Minor LB, Poe D, editors. Glasscock-Shambaugh Surgery of the Ear. Trans. from English; Karpichenko SA, Editor. 6th ed. PH Panfilov: Moscow; 2015. 864p

[2] Bronstein A, Lempert T. Dizziness (A Practical Approach Diagnosis Management). Trans. from English; Parfenova VA, Editor. GEOTAR-media: Moscow; 2010. 216p

[3] Veber P, editor. Vertigo and Disequilibrium: A Practical Guide to Diagnosis and Management. 2nd ed. New York: Thieme; 2017. 232p

[4] Brandt T, Dieterich M, Strupp M. Vertigo and Dizziness. Common Complaints. Trans. from English; Timonina N, Editor. Moscow: Practica; 2009. 200p

[5] Jáuregui-Renaud K, GutierrezMarquez A, Viveros-Rentería L, Ramos-Toledo V, Gómez-Alvarez F. Neurotology symptoms at referral to vestibular evaluation. Journal of Otolaryngology - Head \& Neck Surgery. 2013;42(1):55. DOI: 10.1186/1916-0216-42-55

[6] Babiyak VI, Lantsov AA, Bazarov VG. Clinical Vestibulology: A Guide for Doctors. St. Petersburg: Gippokrat; 1996. 332p

[7] Purkyně E. On the forms of the cells of the anthers, with fibrous and also on the grains, pollinarium: commentary on phytochemical. J.D. costs Grusonii; 1830. $112 \mathrm{p}$

[8] Flourens P, Flourens P-M-J, Rolando L. Recherches Expérimentales Sur Les Propriétés Et Les Fonctions Du Système. Chez Crevot; 1824. 384p

[9] Evald JR. Physiologische Untersuchungen Bber Das Endorgan
Des Nervus Octavus Wiesbaden. Germany: Bergman; 1892

[10] Babiyak VI, Hoffman VR, Nakatis YK. Neurootorinolaryngology: A Guide for Doctors. Gippokrat: St. Petersburg; 2002. 726p

[11] Babiyak VI, Yanov YK. Vestibular Functional System.St. Gippokrat: Petersburg; 2007. 426p

[12] Blakley BW, Chan L. Methods considerations for nystagmography. Journal of Otolaryngology - Head \& Neck Surgery. 2015;44(25). DOI: 10.1186/s40463-015-0078-2

[13] Kim HA, Bisdorff A, Bronstein AM, Lempert $\mathrm{T}$, et al. Hemodynamic orthostatic dizziness/vertigo. Diagnostic Criteria. 2019;29(2-3):45-56. DOI: 10.3233/VES-190655

[14] Cohen HS. A review on screening tests for vestibular disorders. Journal of Neurophysiology. 2019;122(1):81-92. DOI: 10.1152/jn.00819.2018

[15] Zhou YF, Wang ZL, Zheng HZ, Zhang HW, et al. Application of modified Dix-Hallpike maneuver in the diagnosis of posterior semicircular canal benign paroxysmal positional vertigo. Lin Chung Er Bi Yan Hou Tou Jing Wai Ke Za Zhi. 2019;33(6):512-514. DOI: 10.13201/j.issn.1001-1781.2019.06.009

[16] Imai T, Okumura T, Sato T, Takeda N, et al. Effects of interval time of the Epley maneuver on immediate reduction of positional Nystagmus: A randomized, controlled, non-blinded clinical trial. Frontiers in Neurology. 2019;10:304. DOI: 10.3389/ fneur.2019.00304

[17] Gupta AK, Sharma KG, Sharma P. Effect of Epley, Semont maneuvers and Brandt-Daroff exercise on quality of life in patients with posterior semicircular 
canal benign paroxysmal positional vertigo (PSCBPPV). Indian Journal of Otolaryngology and Head \& Neck Surgery. 2019 Mar;71(1):99-103. DOI: 10.1007/s12070-018-1322-7

[18] Tavartkiladze GA. Guidelines for clinical audiology. Moskva: Meditsina; 2013. 676p

[19] Lopez-Escamez JA, Dlugaiczyk J, Jacobs J, Lempert T, et al.

Accompanying symptoms overlap during attacks in Menière's disease and vestibular migraine. Frontiers in Neurology. 2014;5:265. DOI: 10.3389/ fneur.2014.00265

[20] Mischanchuk N, Sushro Y, Buzunov V, Fedirko PH. Sensory organs. In: Cerdiuk A, Bebeshko V, Bazyra D, Yamashita S, editors. Chapter 18. Health Effects of the Chornobyl Accident-A Quarter of Century Aftermath. KyivNagasaki: DIA; 2011. pp. 491-532

[21] Zabolotnyi DI, Mishchanchuk NS. Vestibular and acoustic dysfunctions in clean-up workers of Chornobyl accident (30 years of follow-up). Problemy Radiacijnoyi Medytsynyi Radiobiologii. 2016;21:218-237. (in Ukrainian)

[22] Barany R, Ibershoff AE. Physiology and Pathology of the Semicircular Canals; Being an Excerpt of the Clinical Studies of Dr. Robert Barany with Notes and Addenda Gathered from the Vienna Clinics. New York: P. B. Hoeber; 1910. 66p

[23] Semmanaselvan K, Vignesh SS, Muthukumar R, Jaya V. Vestibular evoked myogenic potentials after Epleys manoeuvre among individuals with benign paroxysmal positional vertigo. Indian Journal of Otolaryngology and Head \& Neck Surgery. 2019 Jun;71(2):195-200. DOI: 10.1007/ s12070-019-01581-6 
Section 2

Motor Research 



\title{
Examination of New Parameters from F-Wave Waveform Using Addition Averaging Method
}

\author{
Marina Todo
}

\begin{abstract}
To evaluate the excitability of the spinal motor neural function, $\mathrm{F}$ wave in evoked electromyogram is often used. As the dominant nerve to the muscle was electrically stimulated, retrograde action potentials are transmitted from the stimulation point to the anterior horn cells of the spinal cord. Action potentials that are regenerated in the anterior horn cells of the spinal cord are transmitted to the muscle in an anterograde manner, which are recorded at the muscle. From this route, because $\mathrm{F}$ wave can be measured from the whole body nerve, it has been used in various situations. Usually, it is characteristic that the waveform of $F$ wave appears in variety. In other words, the same waveform does not appear in healthy people. However, the same waveform may appear when a certain disease occurs. It has been reported that the same waveform called "repeater F" appears in patients with neurological diseases. However, techniques and experience are required to analyze the waveform, and it is not widely used in clinical practice. This article will explain the $\mathrm{F}$ waves in terms of neurophysiology and investigate whether the averaging method can be applied to F-wave waveform analysis.
\end{abstract}

Keywords: F-wave waveform, usefulness of the addition averaging method, correlation coefficient, same waveform, F-wave parameters

\section{Introduction}

When we move, the electrical signals are generated in the brain through the spinal cord, and muscles contract, which causes movement. In the electrical signal transmission, although the function of the spinal cord is important, there is no consensus on the function (i.e., spinal cord excitability). The F wave which is an evoked electromyogram is one of the means to reflect the excitability of the spinal cord. Although the generation mechanism of the $\mathrm{F}$ wave will be described later, the $\mathrm{F}$ wave is characterized by various waveform shapes that can be usually recorded. On the one hand, in cases of spinal cord disease and cerebral infarction, the $\mathrm{F}$ wave with same shape may be recorded. Recently, due to these characteristics of the $\mathrm{F}$ wave, the excitability of the spinal cord has been utilized for grasping the pathological condition of neurological disorders. On the other hand, the analysis for the waveform of the $\mathrm{F}$ wave varies depending of the researcher, and the $\mathrm{F}$ wave appears in various ways; it has not yet been established as one evaluation. Hence, the ultimate objective is to establish an analysis method of waveform with low cost 
and effort and to improve the versatility of the analysis method in clinical setting. I will explain the $\mathrm{F}$ waves in terms of neurophysiology and investigated whether the averaging method can be applied to F-wave waveform analysis. In this article, we will explain with a brief reference to the physiological aspects of the F-wave generation mechanism in order to understand waveform analysis more deeply. The same waveform appears in motor nerve demyelinating diseases and diseases involving degeneration of spinal anterior horn cells. However, the same criterion for waveforms may differ from each researcher. Therefore, we found the same criterion using the correlation coefficient. The usefulness of the addition averaging method in waveform analysis was examined using the reference value of the same waveform and will be introduced here.

\section{Outbrealk mechanism and characteristics of $F$ wave}

F waves conduct retrogradely toward the center when a certain electrical stimulus is afforded to the $\alpha$ motor neuron (hereinafter $\alpha \mathrm{MN}$ ). Originally, the axon does not reach the cell body due to impedance mismatch (resistance $\Omega=\mathrm{V} / \mathrm{I}$ ) at the axion hillock. Therefore, the spinal cord anterior horn cells do not refire and do not generate $\mathrm{F}$ waves. However, when spikes cross the axion hillock and reach the anterior horn cells of the spinal cord due to some effect, they propagate to the dendrites and generate SD (soma-dendritic) spikes there. This spike reaches the axion hillock that has escaped from the absolute refractory period and conducts the axon antegrade from there, resulting in compound muscle action potential (CMAP) evoked from the governing muscle [1]. Nerve fibers have an absolute refractory period of $1 \mathrm{~ms}$, and if the spike returns during the period corresponding to the refractory period of the axion hillock, no F waves can be generated because they cannot be conducted antegrade. Also, $\alpha \mathrm{MN}$ has a structure of antidromic inhibition via Renshaw cells. Antidromic inhibition takes $2 \mathrm{~ms}$, and it takes $1 \mathrm{~ms}$ to return from the axion hillock to the spinal cord anterior horn cells. In other words, in order to generate the $\mathrm{F}$ wave, the spinal cord anterior horn cells must reignite and conduct to the periphery between $1 \mathrm{~ms}$ after the absolute refractory period of the axion hillock has ended and $2 \mathrm{~ms}$ before antidromic inhibition of Renshaw cells. Whether or not the axion hillock is out of the absolute refractory period is determined by slight timing differences, and this is thought to significantly affect the incidence of $F$ waves $[1,2]$. In addition, the following three conditions are considered in which the F wave is likely to occur: (1) the hyperpolarized state in the cells increases the time required for conduction to the dendrites, which makes it easier to avoid the absolute refractory period of the axion hillock; (2) reduction of the absolute refractory period; and (3) excessive depolarization of the axon lowers the threshold level, making spikes more likely to occur with small stimuli [1] (Figure 1).

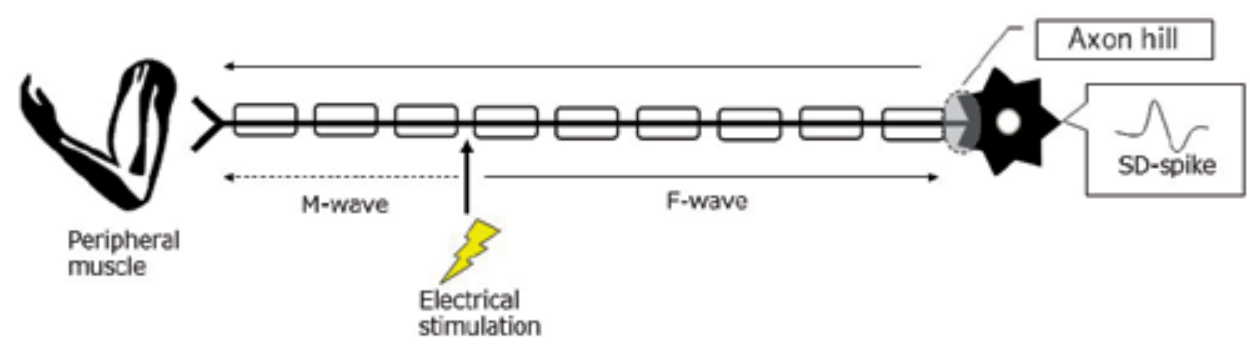

Figure 1.

F-wave generation mechanism. 
Although the $\mathrm{F}$ wave is conducted antegrade by refiring of the spinal cord horn cells and can be recorded, refiring does not occur in all neurons. Furthermore, the probability of firing the same cell with each electrical stimulation is usually low, and re-excitation occurs only once every 10-100 times [1]. Komori et al. reported that $1406 \mathrm{~F}$ waves were evoked by a total of 1957 electrical stimuli in 10 cases, which consisted of 1160 waveforms, of which 1033 (89.1\%) had only 1 occurrence [3]. F waves can be evoked from most motor nerves, but the nerves used mainly for examination are the median nerve, ulnar nerve, and tibial nerve. Among these nerves, the ulnar and tibial nerves have a persistence close to $100 \%$. In addition, the normal value of the F-wave persistence varies among researchers. For the median nerve, Komori and Suzuki et al. reported more than $40 \%$, and Fisher et al. reported 79-100\%, but if too high, they may suspect spasticity, a disorder of upper motor neurons. There are reports of peroneal nerves in the nerves targeted for F-wave tests, but it is said that there are cases of difficulties in Japanese people even in healthy subjects $[4,5]$. In the background, Japanese people are suspected of having a potential peroneal neuropathy due to the unique Japanese culture such as sitting straight custom. Hirashima et al. reported that CMAP (M-wave) amplitude increased by peroneal nerve stimulation since 1995 [6] and examination of the peroneal nerve is now being examined.

\section{Relationship between $\mathrm{F}$ wave and motor unit}

In the anterior horn of the spinal cord, there are neurons of various motor units (motor neuron pool). A motor unit is a unit composed of motor neurons, and the muscle fibers are governed by the neurons. One F wave appears due to the combination of refiring motor units. The F-wave waveforms are different due to the combination of the motor units and the timing shift; therefore, the F wave has a feature in which its waveforms appear in a variety. There are three types of motor units. The $\mathrm{S}$ type (slow-twitch type) is small in size and slow in contraction speed. The FF type (fast-twitch fatigable type) is large in size and fast in contraction speed but is easily fatigued. The FR type has characteristic between $S$ type and FF type. When a person exerts, it depends on the number of motor units activated (recruitment) and the frequency of firing of motor neurons (rate coding) [7].

Henneman reports that there is a strong correlation between motor neuron size and recruitment order [8]. When exercising, there are rules that are mobilized in order from smaller motor units to larger motor units (size principle) [1, 2, 7]. Small motor neurons have a low stimulus threshold and are easy to fire even with weak input. The percentage of motor units varies from muscle to muscle. Enoka reported that $92.5 \%$ of S-type motor neurons and $7.5 \%$ of FR-type motor neurons dominated in gastrocnemius muscles. Similarly, the musculus interossei dorsales pedis and triceps brachii muscle were dominated mostly by small motor neurons [9].

When humans exert their power, fluctuations in rate coding, in addition to recruitment of motor units, have a great effect, too $[10,11]$. For example, in a study by Moritz et al., as the muscle contraction strength of the musculus interossei dorsales pedis of the hand was gradually increased, the rate coding of firing and the number of motor units increased, and the contraction strength reached $60 \%$ or more (against maximum contraction strength). At $60 \%$ or more contraction, most of the work was done only by increasing the rate coding. In the case of $\mathrm{F}$ wave, it has no effect on one waveform, but $F$ wave treats the average value of the waveform obtained by 16 or more stimuli. At that time, the rate coding of the same motor unit with respect to the number of stimulations is reflected [12]. 
From the above, it is necessary to adjust the rate coding of firing of units of various sizes according to the scene. When various units fire, they appear in the waveform of the $\mathrm{F}$ wave, and the rate coding is directly reflected in the persistence of the $\mathrm{F}$ wave. In other words, it can be said that the waveform and appearance persistence of the $F$ wave reflect the motor unit. However, it has been reported that when the number of anterior horn cells decreases or degenerates due to spinal cord anterior horn cell disease or motor neuron damage, the firing of the same unit is biased and the $F$ wave shows the same waveform. Evaluation the $\mathrm{F}$ wave reflecting the unit this way has the potential to lead to an objective evaluation of muscle output and voluntary motion.

\section{F-wave parameters}

\subsection{Latency (ms)}

Latency is the time taken of an electrical stimulation conduction to reach the spinal cord anterior horn cells and return to the dominant muscle. This parameter is most commonly used in clinical practice and is a highly reproducible index. In addition, the shortest rise latency in the recorded $\mathrm{F}$ wave is called the minimum latency, which reflects motor fibers with fast conduction time. Conversely, the longest rise latency is called the maximum latency, which reflects motor fibers with slow conduction times (Figure 2). The speed of conduction is said to be affected by height, limb length, and skin temperature. Speed of conduction is particularly reduced in diseases that affect the myelin of peripheral nerves (e.g., Guillain-Barre syndrome, Charcot-Marie-Tooth disease, etc.) [1].

\subsection{Amplitude $(\mu \mathrm{V})$}

Amplitude represents the magnitude of the waveform. There are two types of measurement methods. The first is the baseline to peak amplitude. The second is the peak-to-peak amplitude of the negative and positive vertices (Figure 3). We used

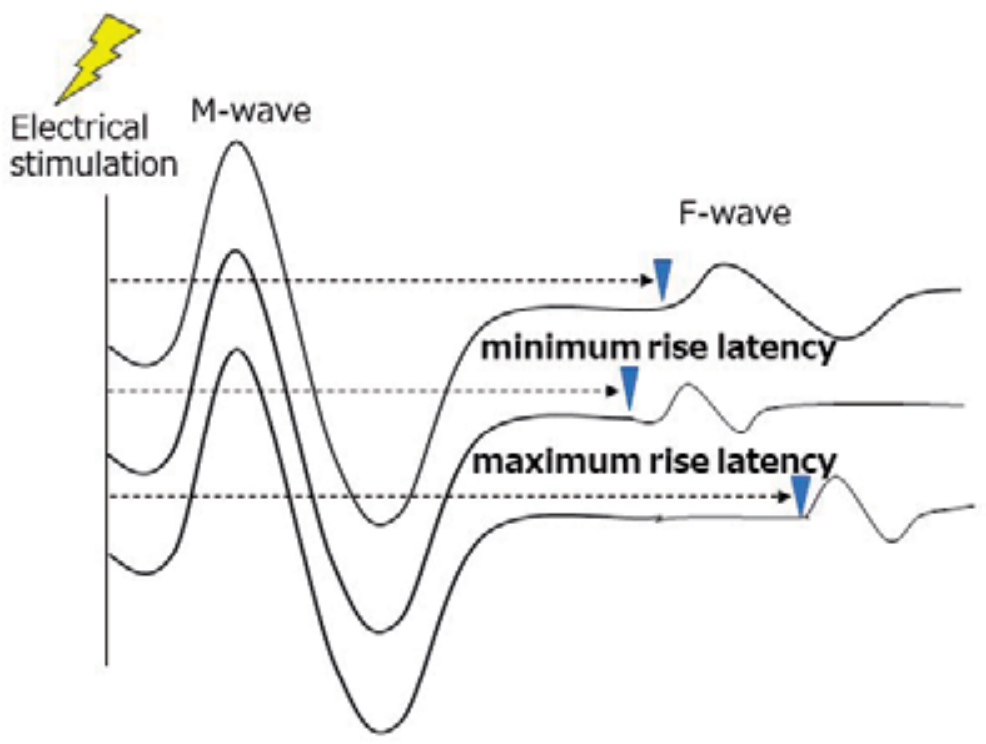

Figure 2.

Latency type. 


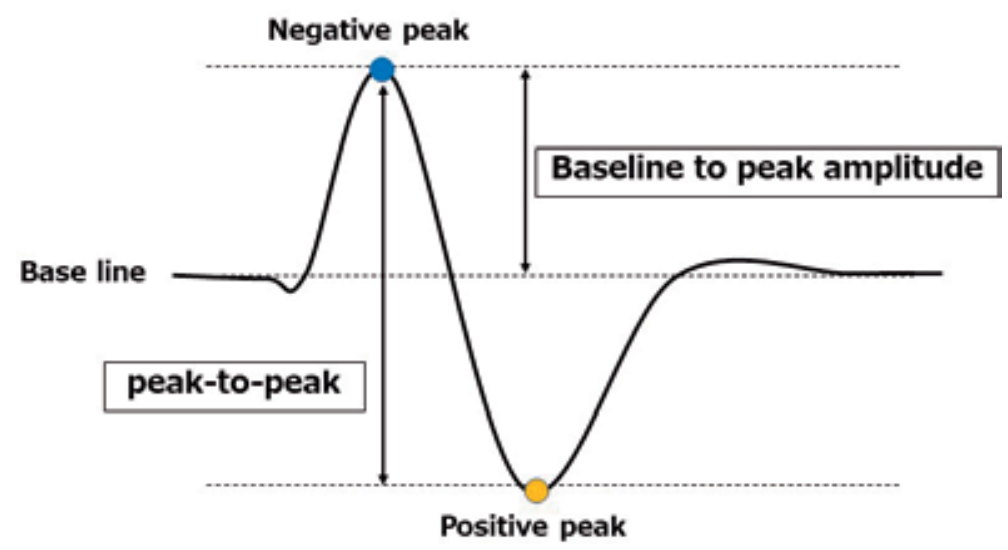

Figure 3.

A way to measure amplitude value.

the second peak-to-peak amplitude this time to analyze the waveform, including the positive wave. To normalize the amplitude of the $\mathrm{F}$ wave, the amplitude $\mathrm{F} / \mathrm{M}$ ratio is expressed by the ratio divided by the peak-to-peak amplitude of the $M$ wave when the maximal stimulation is given to the motor nerve. It is said that if the amplitude $\mathrm{F} / \mathrm{M}$ ratio shows a value of $5 \%$ or more, the possibility of upper motor neuropathy is suspected [13].

\subsection{Duration (ms)}

Duration indicates the time from the rise of the amplitude of the $\mathrm{F}$ wave to its return to the baseline. However, depending on the waveform, the position to return to the baseline is unclear, and care must be taken when using this index because it may be affected by the experience value of the measurer. In the case of the median nerve, the location where the $F$ wave is generated is on the baseline after the generation of the $\mathrm{M}$ wave, so it is difficult to define the baseline (Figure 4).

\subsection{Persistence (\%)}

Persistence indicates the ratio of the appearance of the $\mathrm{F}$ wave to the total number of stimulations. Analysis of only one waveform is not sufficient because

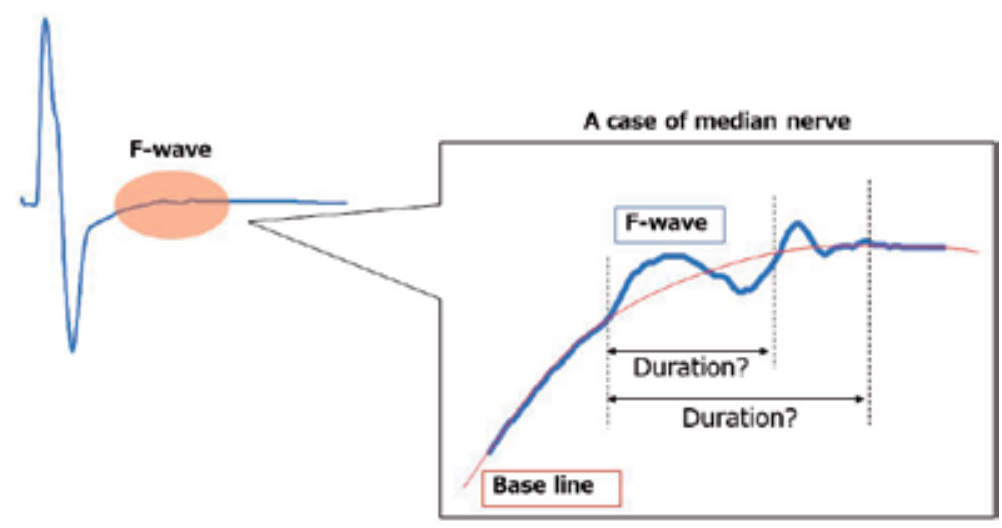

Figure 4.

Cases where it is difficult to measure the duration of the median nerve. 
the $\mathrm{F}$ wave has different waveforms at each stimulus. Komori et al. reported that sufficient reproducibility was obtained with more than 50 stimuli [14]. Currently, Kimura et al. have generally analyzed using the average value of $\mathrm{F}$ waves obtained at least 16-30 times or more stimuli.

Others analyze the number of negative vertices of the waveform. There is also an index called Fchronodispersion which indicates the variation of latency from the difference between the minimum latency and the maximum latency.

\section{Characteristics of disease and F-wave waveform}

Among peripheral neuropathies (neuropathies), abnormalities of F-wave waveform due to motor nerve disorders have been reported mainly by Kimura et al. [1, 15-19]. There are reports of Guillain-Barre syndrome [15-17], Charcot-Marie-Tooth disease [19], and diabetic neuropathy [20, 21], which are diseases in which motor nerves are predominantly impaired. There are reports related to spasticity, one of the pyramidal tract disorders [22-25]. In recent years, there have been many reports of amyotrophic lateral sclerosis that is an intractable disease in which both upper and lower motor neurons are degenerated $[1,2]$. In neuropathy, demyelination of axon myelin sheaths inhibits jump conduction and slows the conduction speed. As a result, it is easy to obtain a result such as a delay in the rise latency and a decrease in the persistence of the $\mathrm{F}$ wave. These diagnoses can be determined by latency which has high reproducibility among F-wave parameters.

The authors focused on abnormal $\mathrm{F}$ waveforms in disorders involving upper motor neuron disorders and spinal cord anterior horn cell degeneration. In cases of spasticity among stroke, Suzuki et al. report that as the electrical stimulation intensity is increased, the waveform of the $\mathrm{F}$ wave appears more like the $\mathrm{H}$ wave as the degree of muscle tone and tendon reflex increases [26]. In addition, Suzuki et al. measured $\mathrm{F}$ waves obtained from the abductor of the thumb by median nerve stimulation in one case of cerebrovascular disease and compared the disease duration at 9 months, 52 months, and 70 months later. Meanwhile, physical therapy was given. As a result, they reported that as the duration of physiotherapy increased, the amplitude of $F$ waves decreased, and the waveforms varied. In addition, improved hand muscle tone and voluntary motion. Komori et al. also reported that the amplitude of $F$ waves increased with spasticity [25]. However, other researchers measured $\mathrm{F}$ waves when median nerve stimulation was applied to 14 stroke patients, and the latency and persistence were not significantly different between the non-paralyzed side and the paralyzed side. The persistence of motor unit has been reported to decrease significantly on the paralyzed side regardless of the degree of muscle tone $[23,27]$. In the case of amyotrophic lateral sclerosis, atrophy of the anterior horn cells of the spinal cord reduces the number of motor units and the number of firing anterior horn cells, so the persistence decreases significantly, and the same waveform begins to appear [27]. Similarly, spinal and bulbar muscular atrophy (SBMA) has been reported to produce F-wave waveform with similar characteristics [28-30].

\section{Regulation of the same waveform}

F waves with matching latency and amplitude values may appear when spasticity due to stroke or when atrophy of the spinal cord horn cells occurs, such as in ALS and SBMA. This is known as "repeater F." This repeater F is reported 
to significantly appear more in patients with diabetic polyneuropathy, ALS, carpal tunnel syndrome, ulnar neuropathy, and L5 radiculopathy than in healthy subjects $[1,2,31,32]$.

The authors wondered how it is difficult to determine the consistency of the waveforms using only the amplitude value or the latency and how to judge the waveforms to be the same. Some studies report the same waveform as an analysis item, but few describe the judgment method in detail. Some of the methods described for determining the same waveform include the following: (1) a method of visual judging by superimposing recorded waveforms [3, 33], (2) a method of determining the correlation coefficient between waveforms at a value of 1.00 $[34,35]$, and (3) in recent years, some researchers have decided to develop their own analysis software [36]. There is a risk that the evaluation method can be used only by those who are familiar with $\mathrm{F}$ waves when using the visual observation to determine the same waveform, because the degree of match defined as the same waveform includes individual differences.

\subsection{Regulation of the same waveform using correlation coefficient}

The authors examined the relationship between the results that three researchers familiar with $\mathrm{F}$ waves judged visually the same waveform and the results of the correlation coefficient calculated from the data [37]. The target waveform is a waveform of 30 shots derived from the musculus abductor pollicis brevis by median nerve stimulation of a healthy person for 5 trials. A total of 150 waveforms were prepared. We asked each researcher to select the same waveform in a separate room to avoid interference. The data processing method of the $\mathrm{F}$ wave was a moving average of three terms using Microsoft Excel. The correlation between the waveform after the moving average and the raw waveform was an extremely high value of $\mathrm{R}=0.9963$, which sufficiently reflects the raw waveform. Next, the correlation coefficients of all combinations of waveforms evoked by applying 30 electrical stimuli per trial were calculated. The waveforms visually judged the same waveforms selected by two or more out of three people.

The results showed that the same waveform was selected by the 2 individuals as 10 waveforms, of which 9 (90\%) showed a correlation coefficient of 0.95 or more. Next, the waveforms selected by the three persons had two waveforms, and both waveforms showed a correlation coefficient of 0.95 or more. It was suggested that the number of correlation coefficients $R=0.95$ may be one index of the same waveform when analyzing the waveform of the $\mathrm{F}$ wave (Table 1 ).

However, one waveform judged to be the same waveform was recognized, although the correlation coefficient was 0.71 . They were similar in waveform

\begin{tabular}{|c|c|c|c|c|c|}
\hline & \multicolumn{3}{|c|}{ _ Correlation coefficient } & -1 & \multirow[b]{2}{*}{$\begin{array}{l}\text { Total number } \\
\text { checks }\end{array}$} \\
\hline & 0.95 or more & $0.95 \sim 0.90$ & $0.89 \sim 0.85$ & 0.85 or less & \\
\hline $\begin{array}{l}\text { Zperson } \\
\text { Max:1.00 } \\
\text { Min:0.71 }\end{array}$ & $9(90 \%)$ & orom; & $010 \% i$ & $1: 10 \%$ & 10 \\
\hline $\begin{array}{l}\text { 3person } \\
\text { Max:0.99 } \\
\text { Miา:0.9: }\end{array}$ & $20100 x$ & aroms & alosi & $9(0 \%)$ & 2 \\
\hline
\end{tabular}

Table 1.

Results of the selection of the same waveform. 
but slightly different in latency when the actual raw waveforms were checked. Therefore, the latency was shifted by $0.625 \mathrm{~ms}$ until the negative peak matches with the visual confirmation; as a result the waveform showing a correlation coefficient of 0.71 showed a correlation coefficient of 0.96 .

Although the waveforms were the same in this study, the different latencies suggested that the correlation coefficient was low. We thought that the slight shift in the rise latency was affected the time it took for the spike to occur in the spinal cord anterior horn excitation threshold. This time the evoked musculus abductor pollicis brevis is composed of 115-171 motor units, considering the possibility that the rise latency could be shifted due to the spatial relationship of the cell bodies when cells are similar in size during refiring. Komori et al. allow for differences in negative peak latencies up to $1 \mathrm{~ms}$ when comparing the reproducibility of $\mathrm{F}$ waves [38]. It may be necessary to consider such differences in latency when analyzing the same waveform.

\section{Research using the addition averaging method}

An analysis method that takes into account the $\mathrm{F}$ wave, in which various waveforms appear, has not yet been established. In addition, there is a report on the F-wave method (F-MUNE) for estimating motor units using F waves. However, analysis requires time and effort, and versatility is poor at present $[27,39,40]$. Therefore, as stated in this introduction, we examined whether the averaging method is applicable to $\mathrm{F}$ waves to increase versatility by establishing simple analysis methods. The averaging method approaches 0 by averaging the waveforms that appeared in diversity with repeated stimuli. On the other hand, a waveform that appears at a fixed latency also appears as a waveform after overlapping and addition averaging [1]. In the case $\mathrm{F}$ wave appears in the diversification, the value become close to 0 when the addition averaging process is performed. The wave remains after the addition averaging process when the same waveform appears repeatedly. It may be possible to distinguish the same or similar waveforms if the addition averaging method is used to analyze the waveform of the $\mathrm{F}$ wave. However, there is a risk that the waveform may disappear due to a slight difference in latency when applying this addition averaging method to the analysis of the waveform of the $F$ wave. Furthermore, meaning is still unclear what waveform is calculated by the addition averaging method, so we first examined the relationship between the averaging waveform and the raw waveform.

\subsection{Previous research on waveform analysis using the addition averaging method}

Eisen et al. [13] first studied $\mathrm{F}$ waves using the addition averaging method. They addition averaged the $\mathrm{F}$ waves obtained by giving 32 electrical stimulations into the tibial nerve of cerebrovascular patients and compared them with that of the healthy subjects. The waveform after addition averaging reported that the duration and amplitude could be reproduced in both cerebrovascular and healthy subjects [13]. In Japan, Komori et al. focused on the latency of the negative peak in order to pursue an evaluation method that combines reproducibility and simplicity in order to promote clinical application of $\mathrm{F}$ wave. The waveform after adding the negative peaks manually (addition method) showed that the reproducibility of the waveform was $86.7 \%$ for the median nerve and $73.3 \%$ for the tibial nerve, even when measured on another day [38]. Next, they reported that the waveform obtained by the addition averaging method sufficiently reflected the waveform of the proven addition method [41]. Sakamaki et al. point out that such as F waves, when negative waves 
and positive waves appear at the same latency, may be offset when the addition averaging method is used for waveforms that appear in diversity [42]. Hiratsuka et al.'s research also tried to use the addition averaging method, but the amplitude was significantly reduced, making analysis difficult [43]. There have been fewer studies using the addition averaging method for $\mathrm{F}$-wave analysis since the first half of 1988 from this background. There are other reports that use the addition averaging method for the F-wave analysis items [44-46], but only the latency and duration reflect the raw waveform, and it is a difficult situation to establish these as parameters.

\subsection{Relationship between F-wave persistence and addition averaging waveform}

We reexamined the usefulness of the addition averaging method, taking into contents what Sakamaki pointed out. First, we examined whether the persistence of $\mathrm{F}$ waves needs to be considered for the median nerve since the persistence of $\mathrm{F}$ waves differs for each nerve to be affected.

The method measured the $\mathrm{F}$ wave at rest and calculated the coincidence rate between addition averaging waveform for all waveforms and averaging waveform reflecting only F-wave appearance waveform. Subjects were 99 healthy volunteers (55 males and 45 females) who agreed to the study with an average age of $23.3 \pm 5.3$ years. The $\mathrm{F}$ wave was obtained by electrically stimulating the median nerve of the non-dominant upper limb. From the measured $\mathrm{F}$ waves, the waveform after addition average of the "waveform data of 1 trial (30 shots) (thereafter, TW)" and "waveform data of only the F-wave appearance waveform (thereafter, AW)" was obtained using Microsoft Excel. Next, the correlation coefficient between the two waveforms after addition average values was calculated using CORREL function. This correlation coefficient indicates the coincidence rate. The reference value of the same waveform used the correlation coefficient of 0.95 from previous studies. Next, a scatter diagram from the data of the F-wave persistence of each subject is created, and the coincidence rate is calculated using single regression analysis. The coefficient of determination (R2) is calculated, and the variation is obtained. It is examined whether the coincidence rate depends on the persistence of $\mathrm{F}$ wave. 80/99 subjects had a correlation coefficient between TW and AW of 0.95 or more, accounting for $80 \%$ of the total. Among them 38 subjects showed a correlation coefficient of 1.00 , representing $38 \%$ of the total. $19 / 99$ subjects showed a correlation coefficient of

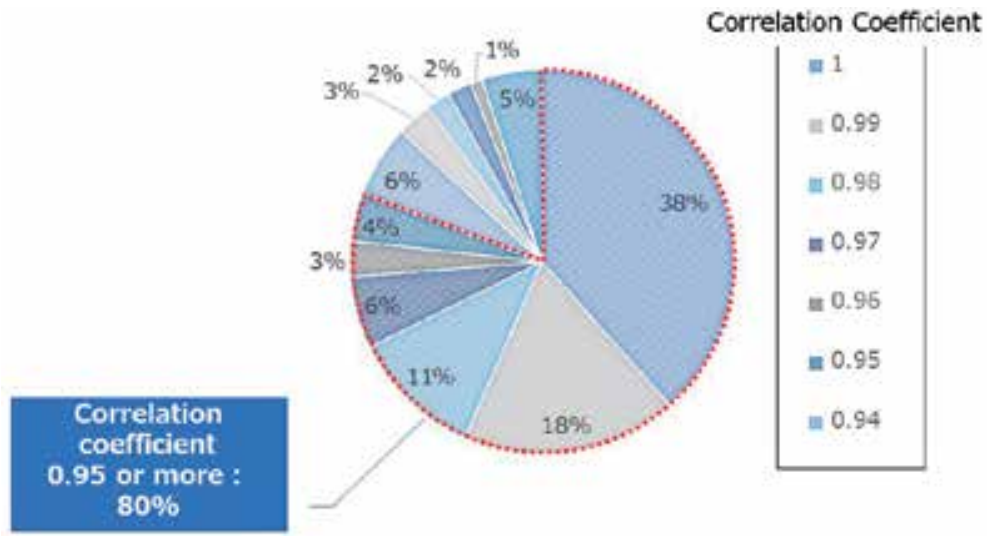

Figure 5.

A circle graph showing the correlation coefficient of two addition averaging waveforms. 


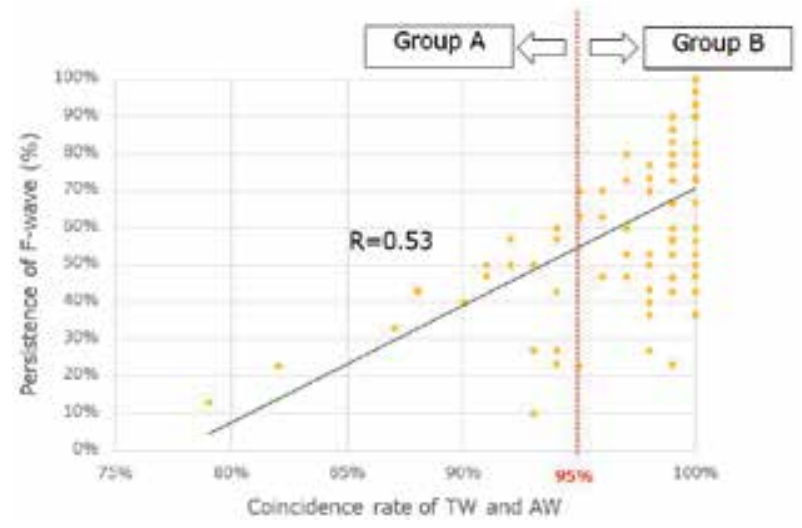

Figure 6.

A scatter plot showing the persistence of $F$ wave and the coincidence rate.

0.95 or less, accounting for $20 \%$ of the total (Figure 5). A scatter plot showing the persistence of $\mathrm{F}$ wave and the coincidence rate is presented in Figure 6.

The coefficient determination $(\mathrm{R})$ was 0.53 , indicating "somewhat correlated." The highest $\mathrm{F}$-wave persistence was $60 \%$, and the lowest value was $10 \%$ in the group that showed a concordance rate of 0.95 or less (thereafter, group A). The highest F-wave persistence was $100 \%$, and the lowest value was $23 \%$ in the group that showed a concordance rate of 0.95 or higher (thereafter, group B) (Figure 6). The result we expected was that the higher the persistence of $\mathrm{F}$ waves, the higher the coincidence rate. However, those with a high coincidence rate and those with a low coincidence rate were recognized despite low persistence of $\mathrm{F}$ wave in group $\mathrm{A}$ and group $\mathrm{B}$. Therefore, we confirmed the raw waveform of those with low persistence of $F$ wave in both groups. Then, there were two factors that reduced the coincidence rate in Group A.

The first factor is that the persistence of $\mathrm{F}$ waves is significantly lower. The amplitude could be confirmed visually because the fewer the number of waveforms that appeared, the smaller the value to be divided. On the other hand, the amplitude value was lost because the denominator to be divided was large. From the above, it is considered that the coincidence rate of the two waveforms has a low value. This result is similar to the reported study of Hiratsuka et al. [43].

The second factor is that high-amplitude waveforms appear localized. It was considered that the addition averaging waveform of AW had a higher amplitude value and a lower coincidence rate because of appearing several high-amplitude waveforms. In group $\mathrm{B}$, there were also two possible factors for the high coincidence rate despite the low persistence of $\mathrm{F}$-wave appearance as in group $\mathrm{A}$. The first factor is that the persistence of the $\mathrm{F}$ wave is around $50 \%$, and the appearing waveform shows almost a similar waveform. The second factor is, even though the number of appearing waveforms was few, they were canceled out by the appearance of diversity which is also a characteristic of the $\mathrm{F}$ wave. As a result, we thought that the matching rate approached the baseline and showed a high value. We think that the tasks of making the persistence of $\mathrm{F}$ waves approach $100 \%$ using muscle contraction or evoked from tibial nerve are suitable when using addition averaging method to analyze F-wave waveform.

\subsection{Method of addition averaging}

\subsubsection{Normal or match of negative peak or match of rise latency}

Compared with the commonly used amplitude F/M ratio, the amplitude value of after addition averaging waveform showed about $1 / 2$ of the value [41]. It is 


\begin{tabular}{|c|c|c|c|c|}
\hline & \multirow[b]{2}{*}{$\begin{array}{l}\text { Common } \\
\text { values }\end{array}$} & \multicolumn{3}{|c|}{ Addition averaging } \\
\hline & & Normal & $\begin{array}{l}\text { Match of } \\
\text { negative peak }\end{array}$ & $\begin{array}{l}\text { Match of ilse } \\
\text { latency }\end{array}$ \\
\hline Meseu'ed value $(a v)$ & $185,9 \overline{7}$ & $\begin{array}{l}-E 0.13 \\
(-75.79)\end{array}$ & $\begin{array}{l}146.38 \\
(-39.59)\end{array}$ & $\begin{array}{l}123.92 \\
(-67.05)\end{array}$ \\
\hline rik amolizde rajo ins: & 2.00 & $\frac{-7}{(-0.3)}$ & $\begin{array}{l}1.57 \\
(-0.43)\end{array}$ & $\begin{array}{l}1.33 \\
(-1 . .57)\end{array}$ \\
\hline
\end{tabular}

Table 2.

Comparison of three addition averaging methods and average data.

considered to be the result of the overlap between the negative and positive waves as reported by Sakamaki and Takasu [42]. To avoid the reverse winding proposal, averaging was performed in three ways, and the ratio of amplitudes of addition averaging and the average of the values obtained for each stimulus were compared. The three ways of performing the averaging method are described below:

1. A method of addition averaging the recorded $\mathrm{F}$ wave as it is (normal).

2. A method of addition averaging after the match of negative peak.

3. A method of addition averaging after the match of rise latency.

Subjects picked up and analyzed one subject who showed $100 \%$ persistence of $\mathrm{F}$ wave. The results are shown in Table 2. The error amplitude F/M ratio was $2.0 \%$, and the measured value was $25.79 \mu \mathrm{V}$ during normal addition averaging. Next, the error when matching the negative peak was $0.43 \%$, and the measured value was $39.59 \mu \mathrm{V}$. Finally, the error when the rise latency was matched was $0.67 \%$, and the measured value was $62.05 \mu \mathrm{V}$. From the above, in the case of one subject this time, the average is usually the closest to the raw data, but it is necessary to increase the number of subjects and pursue it because it is the result of only one subject.

\section{Conclusions}

At the present time, when using the addition averaging method for the F-wave waveform analysis, (1) the constant criterion of the same waveform using the correlation coefficient should be 0.95 , (2) the persistence of $F$ waves when using the addition averaging method is at least $60 \%$ or more and (3) the normal addition averaging method has less error in the amplitude value compared to the raw data.

However, all the contents described this time are the analysis results of $\mathrm{F}$ waves evoked by electrically stimulating "median nerve." Therefore, we will analyze the ulnar nerve, the tibial nerve, and the peroneal nerve in the same way as described at the beginning and examine the innervated nerves to which the addition averaging method is applicable. In addition, "cluster analysis" will be introduced to avoid pointing out the content of Sakamaki et al. In this analysis method, similar waveforms are collected to create a cluster. By carrying out the cluster analysis before the addition averaging, the similar waveforms can be classified, and the cancelation of the positive wave and the negative wave due to the variation in the latency can be avoided. In addition, you can see how many F-wave clusters make up one trial 
during a case characteristic of F-wave waveform such as stroke with spasticity or ALS with atrophy of spinal cord horn cells.

\section{Acknowledgements}

We are grateful to Dr. Suzuki and laboratory members for their helpful discussions.

\section{Conflict of interest}

Nothing.

\section{Author details}

Marina Todo

Clinical Physical Therapy Laboratory, Kansai University of Health Sciences,

Osaka, Japan

*Address all correspondence to: todo@kansai.ac.jp

\section{IntechOpen}

(C) 2020 The Author(s). Licensee IntechOpen. This chapter is distributed under the terms of the Creative Commons Attribution License (http://creativecommons.org/licenses/ by/3.0), which permits unrestricted use, distribution, and reproduction in any medium, provided the original work is properly cited. (cc) BY 


\section{References}

[1] Kimura J, Kohara N. Nerve Conduction and Electromyography Studies. 2nd ed. Tokyo, Japan: IGAKUSHOIN Ltd.; 2018. pp. 65, 80-83, 90-104. DOI: $10.11477 / \mathrm{mf} .1416100783$ [in Japanese]

[2] Abe T. The characteristics of F-wave in neurological disorders. Japanese Journal of Clinical Neurophysiology. 2018;46(4):175-186. DOI: 10.11422/ jscn.46.175 [in Japanese]

[3] Komori T, Takahashi R, Hirose K, et al. Variability of F-wave shape. Clinical Electroencephalography. 1988;30(1):1-6 [in Japanese]

[4] Jin C, Baba M, Matsunaga M. A right-left difference in motor nerve conduction parameter in healthy young adults. Clinical [in Japanese] Electroencephalography. 2003;45:234-238

[5] Bischoff C, Stalberg E, Falk B, et al. Significance of A-waves recorded in routine motor nerve conduction studies. Electroencephalography and Clinical Neurophysiology. 1996;101(6):528-533. DOI: 10.1016/S0921-884X(96) 96553-4

[6] Hirashima F, Shimizu T, Koike K, et al. Interdecadal changes of nerve conduction studies of lower limb in healthy Japanese subject. Japanese Journal of Clinical Neurophysiology. 2019;47(6):519-525. DOI: 10.11422/ jscn.47.519 [in Japanese]

[7] Roger M. Neuromechanics of Human Movement. Niigata, Japan: Nishimura Co., Ltd.; 2017. pp. 141-151 [in Japanese]

[8] Henneman E. Relation between size of neurons and their susceptibility to discharge. Science. 1957;126(3287):1345-1347. DOI: 10.1126/ science.126.3287.1345

[9] Enoka R, Fuglevand A. Motor unit physiology: Some unresolved issues.
Muscle \& Nerve. 2001;24(1):4-17.

DOI: 10.1002/1097-4598(200101)24: 1<4::AID-MUS13>3.0.CO;2-F

[10] Desmedt J, Godaux E. Fast motor units are not preferentially activated in rapid voluntary contractions in man. Nature. 1977;267:717-719. DOI: $10.1038 / 267717 \mathrm{a} 0$

[11] Person R, Kudina L. Discharge frequency and discharge pattern of human motor units during voluntary contraction of muscle. Electroencephalography and Clinical Neurophysiology. 1972;32(5):471-483. DOI: 10.1016/0013-4694(72)90058-2

[12] Moritz C, Barry B, Pascoe M, et al. Discharge rate variability influences the variation in force fluctuations across the working range of a hand muscle. Journal of Neurophysiology. 2005;93(5):24492459. DOI: $10.1152 /$ in. 01122.2004

[13] Eisen A, Odusote K. Amplitude of the $F$ wave: A potential means of documenting spasticity. Neurology. 1979;29(9):1306-1309. DOI: 10.1212/ wnl.29.9_part_1.1306

[14] Komori T, Hirose K, Tsubaki T. A study of F-wave technique on the number of stimuli and the reproducibility. Clinical Electroencephalography. 1986;28(11):770-774 [in Japanese]

[15] Fraser J, Olney R. The relative diagnostic sensitivity of different $\mathrm{F}$-wave parameters in various polyneuropathies. Muscle \& Nerve. 1992;15(8):912-918. DOI: 10.1002/ mus. 880150808

[16] Kokubun N, Kuwabara S. Electrical diagnosis of Guillain-Barre syndrome. Clinical Electroencephalography. 2013;41(2):103-111. DOI: $10.11422 /$ jscn.41.103. [Translated from Japanese]

[17] Gordon P, Wilbourn A. Early electrodiagnostic findings in 
Guillain-Barré syndrome. Archives of Neurology. 2001;58(6):913-917. DOI: 10.1001/a rchneur.58.6.913

[18] Rajabally Y, Srinivas V. Practical electrodiagnostic value of $\mathrm{F}$-wave studies in chronic inflammatory demyelinating polyneuropathy. Clinical Neurophysiology. 2013;124(1):171-175. DOI: 10.1016/j.clinph.2012.05.019

[19] Christova L, Alexandrov A, Ishpekova B. Peripheral late waves in patients with hereditary motor sensory neuropathy. Electromyography and Clinical Neurophysiology. 1999;39(6):345-348

[20] Kohara N, Kimura J, Kaji R, et al. $\mathrm{F}$-wave latency serves as the most reproducible measure in nerve conduction studies of diabetic polyneuropathy: Multicentre analysis in healthy subjects and patients with diabetic polyneuropathy. Diabetologia. 2000;43:915-921. DOI: $10.1007 /$ s001250051469

[21] Pan H, Jian F, Lin J, et al. $\mathrm{F}$-wave latencies in patients with diabetes mellitus. Muscle \& Nerve. 2014;49(6):804-808. DOI: 10.1002/ mus. 24127

[22] Yamashita A, Suzuki T, Bunno Y. Characteristic appearances of the H-reflex and F-wave in the lower extremity with increased stimulus intensity in subjects with cerebrovascular disease. Rigakuryoho Kagaku. 2013;28(5):563-567. DOI: 10.1589/rika.28.563

[23] Naseri M, Petramfar P, Ashraf A. Effect of motor imagery on the $\mathrm{F}$-wave parameters in hemiparetic stroke survivors. Annals of Rehabilitation Medicine. 2015;39(3):401-408. DOI: 10.5535/ $\operatorname{arm} .2015 .39 .3 .401$

[24] Hara Y. Paralysis due to upper neuron lesion and motor units: Physiological motor unit number decrement on the hemiparetic side in strokes. Clinical Electroencephalography. 2003;45(7):423-428 [in Japanese]

[25] Suzuki T, Bunno Y, Tani M, et al. F-wave waveform values based on the correlation coefficient of each waveform increased following improved voluntary movements in a patient with cerebrovascular disease: A case study with long-term follow-up. International Journal of Neurorehabilitation. 2016;3(2):1000206. DOI: $10.4172 / 2376-0281.1000206$

[26] Suzuki T, Saitoh E, Tani M, et al. Characteristic appearances of the H-reflex and F-wave with increased stimulus intensity in patients with cerebrovascular disease. Electromyography and Clinical Neurophysiology. 2002;42(2):67-70

[27] Hara Y. The lower motor neuron impairment in the central nervous lesion. Japanese Journal of Rehabilitation Medicine. 2000;37(1):43-52. DOI: 10.2490/ jjrm1963.37.43 [in Japanese]

[28] Suzuki K, Katsuno M, Banno H, et al. CAG repeat size correlates to electrophysiological motor and sensory phenotypes in SBMA. Brain. 2008;131(1):229-239. DOI: 10.1093/ brain/awm289

[29] Hama T, Hirayama M, Hara T, et al. Discrimination of spinal and bulbar muscular atrophy from amyotrophic lateral sclerosis using sensory nerve action potentials. Muscle \& Nerve. 2012;45(2):169-174. DOI: 10.1002/ mus.22291

[30] Ibrahim K, El-Abd M. Giant repeater $\mathrm{F}$-wave in patients with anterior horn cell disorders.

Role of motor unit size. American Journal of Physical Medicine \& Rehabilitation. 
1997;76(4):281-287. DOI:

10.1097/00002060-199709000-00006

[31] Mesrati F, Vecchierini M. F-waves:

Neurophysiology and clinical

value. Neurophysiologie Clinique.

2004;34(5):217-243. DOI: 10.1016/i.

neucli.2004.09.005

[32] Maceod W. Repeater F waves: A comparison of sensitivity with sensory antidromic wrist-to-palm latency and distal motor latency in the diagnosis of carpal tunnel syndrome. Neurology. 1987;37(5):773-778. DOI: 10.1212/ wnl.37.5.773

[33] Peioglou-Harmoussi S, Fawcett P, Howe D, et al. F-responses: A study of frequency, sharp and amplitude characteristics in healthy control subject. Journal of Neurology, Neurosurgery, and Psychiatry. 1985;48(11):1159-1164. DOI: $10.1136 /$ jnnp.48.11.1159

[34] Komatsu N, Take N, Takamori K, et al. F-waves of healthy subjects elicited by voluntary movements of different muscle contraction strengths: Consideration of the type of F-wave waveforms. KANSAIRIGAKU. 2016;16:69-77. DOI: 10.11354/jkpt.6.69 [in Japanese]

[35] Suzuki T, Bunno Y, Tani M. et al, A new analysis method of $\mathrm{F}$-waves to obtain "F-wave waveform values". SM Journal of Neurology and Neuroscience. 2016;2(1):1005

[36] Chroni E, Veltsista D, Papapaulou C, et al. Generation of repeater $\mathrm{F}$ waves in healthy subjects. Journal of Clinical Neurophysiology. 2017;34(3):236-242. DOI: 10.1097/ WNP.0000000000000360

[37] Todo M, Suzuki T, Fukumoto Y, et al. Application of F-waveform for the effect of physical therapy relationship between the correlation coefficient of the f-wave waveforms and waveform identification by investigators. International Journal of Neurorehabilitation. 2017;4(4):1000287. DOI: 10.4172/2376-0281.1000287

[38] Komotri T, Takahashi R, Hirose $\mathrm{K}$, et al. The significance of $\mathrm{F}$-wave negative peak for clinical application. Japanese Journal of Electroencephalography and Electronmyography. 1989;17(3):255-262 [in Japanese]

[39] Doherty T, Komori T, Stashuk D, et al. Physiological properties of single thenar motor units in the F-response of younger and older adults. Muscle \& Nerve. 1994;17(8):860-872. DOI: $10.1002 /$ mus. 880170805

[40] Stshunk D, Doherty T, Kassam A, et al. Motor unit number estimates based on the automated analysis of F-responses. Muscle \& Nerve. 1994;17(8):881-890. DOI: $10.1002 /$ mus. 880170807

[41] Komotri T, Takahashi R, Hirose K, et al. F-wave analysis using signal averaging as a simple and reliable method. Japanese Journal of Electroencephalography and Electronmyography. 1990;18(3):293-301 [in Japanese]

[42] Sakamaki S, Takasu T. Clinical and electrophysiological study of patients with prolonged $\mathrm{F}$ wave duration. Clinical Electroencephalography. 1988;30(1):7-11 [in Japanese]

[43] Hiratsuka H, Inoue Y, Tomonaga S, et al. Comparison of $\mathrm{F}$ wave and $\mathrm{H}$ wave on spastic hand. Orthopedics and Traumatology. 1986;35(1):237-239. DOI: 10.5035/nishiseisai.35.237 [in Japanese]

[44] Abe N, Okamoto N, Otani K, et al. A study of $\mathrm{F}$ wave conduction velocity in normal children. Clinical Electroencephalography. 1988;30(8):530-534 [in Japanese] 
[45] Takada T, Baba H, Tobita T, et al.

Evaluation of the reproducibility

of $\mathrm{F}$-wave in relation to

stimulating condition. Clinical

Electroencephalography.

1993;35(5):302-309

[46] Tanaka S, Hachisuka K,

Ogata $\mathrm{H}$. The usefulness of automatic averaging tibial nerve $F$ waves for assessment of spasticity in stroke hemiplegics. The journal of Japan Accident Medical Association.

1995;45(11):739-743 


\title{
Motor Imagery for Neurorehabilitation: The F-Wave Study
}

\author{
Yoshibumi Bunno
}

\begin{abstract}
The immediate enrollment in rehabilitation program and facilitation of the excitability of spinal motor neurons are very important for post-stroke patients. We previously suggested that persistence and the F/M amplitude ratio, indicator of the excitability of spinal motor neurons, were significantly increased during MI. Thus, MI has a greater effect on the excitability of spinal motor neurons. We also indicated that the imagined muscle contraction strength may not affect the excitability of spinal motor neurons. Further, kinesthetic imagery can more facilitate the excitability of spinal motor neurons. However, longer duration of MI may not affect the excitability of spinal motor neurons. Therefore, slight imagined muscle contraction strength may be sufficient to facilitate the excitability of spinal motor neurons, and duration and strategy of imagery should be considered in neurorehabilitation.
\end{abstract}

Keywords: motor imagery, F-wave, imagined muscle contraction strength, duration, strategy, stroke, neurorehabilitation

\section{Introduction}

The excitability of the motor cortex is decreased after stroke along with damage to neural substrates, loss of sensory inputs, and disuse of the affected limb [1]. Further, the amplitude of motor evoked potentials (MEPs), obtained when transcranial magnetic stimulation (TMS) is applied over the primary motor cortex, is decreased [2]. At the spinal level, the excitability of spinal motor neurons is decreased in post-stroke patients during the acute phase $[3,4]$. The excitability of central and spinal neural function is decreased after stroke. The highest level of functional recovery due to neuroplasticity, cortical reorganization, and regeneration occurs at about 4 weeks after stroke, and recovery reaches plateau within 3 months $[5,6]$. Therefore, in post-stroke patients, the immediate enrollment in rehabilitation programs aiming to facilitate the corticospinal excitability, including the excitability of spinal motor neurons, should be important. Indeed, the early initiation of rehabilitation programs can facilitate the recovery of motor function [6-8].

Motor imagery (MI) is a cognitive process creating specific motor actions within working memory without an actual movement [9]. MI allows patients who cannot volitionally perform movements, such as stroke, to mentally practice a motor task. Numerous neurophysiological studies have discussed the effect of MI on the central nervous system by using positron emission tomography, functional magnetic resonance imaging, and near-infrared spectroscopy [10-13]. The primary motor cortex, 
premotor area, supplementary motor area, prefrontal cortex, parietal lobule, cingulate area, cerebellum, and basal ganglia were activated during MI [10-13]. These brain areas were also activated during motor execution (ME), and thus the MI and ME have a common neural network. Further, the MEP amplitude was significantly increased during MI $[2,14,15]$. Therefore, MI can facilitate the excitability of the central nervous system.

However, the influence of MI on the excitability of spinal motor neurons is still unclear. A significant increase of the spinal motor neuron excitability was observed during MI $[16,17]$. Our previous study also demonstrated a significant increase of the spinal motor neurons during MI of isometric thenar muscle activity at 50\% maximal voluntary contraction (MVC) [18]. Conversely, previous studies demonstrated that excitability of spinal motor neurons is not changed during MI $[14,19,20]$. Additionally, Oishi et al. [21] identified three changing patterns (i.e., facilitation, suppression, and no change) in the excitability of spinal motor neurons during MI. These results of previous studies indicated that MI has various effects on the spinal motor neuron excitability. MI includes various components of perception, such as visual, auditory, kinesthetic, proprioceptive, and vestibular. Further, MI incorporates the spatial (e.g., direction and amplitude), temporal (e.g., synchronization and continuously), and dynamic (e.g., muscle tension) information relevant to the motor task. Therefore, the excitability of spinal motor neurons during MI may be different depending on what modality of MI will be chosen. Then, we investigated the excitability of spinal motor neurons during MI under various conditions, specifically imagined muscle contraction strength, duration, and sensory modality. One of the final goals of our group is to find the best conditions for MI to achieve the optimum outcome during the rehabilitation program. In the following sections, we introduce our previous researches and suggest the application of MI to rehabilitation.

\section{The excitability of spinal motor neurons during MI at different imagined muscle contractions}

\subsection{Background and purpose}

Our research group previously reported that the excitability of spinal motor neurons was significantly increased during MI of isometric thenar muscle activity at 50\% MVC [18]. In actual movement, the excitability of spinal motor neurons was increased linearly with muscle contraction strength [22]. If MI and ME share a common neural network, the excitability of spinal motor neurons increases linearly with imagined muscle contraction strength. Firstly, we aimed to investigate the excitability of spinal motor neurons during MI at different imagined muscle contraction strength. The excitability of spinal motor neurons during MI was assessed using the F-wave [23-26].

\subsection{About the F-wave}

The F-wave is compound action potentials resulting from the re-excitation (backfiring) of spinal anterior horn cells by an antidromic impulse following distal electrical stimulation of $\alpha$ motor neurons [27-29] (Figure 1). The F-wave amplitude was significantly increased when the corticospinal descending volley collides with the antidromic peripheral volley [30]. Therefore, the F-wave is considered to be a probe of the excitability of spinal motor neurons. Further, Rossini et al. [31] suggested that the $\mathrm{F}$-wave is a reliable index of the excitability of spinal motor neurons, even when motor output is extremely minimal, as during MI. 


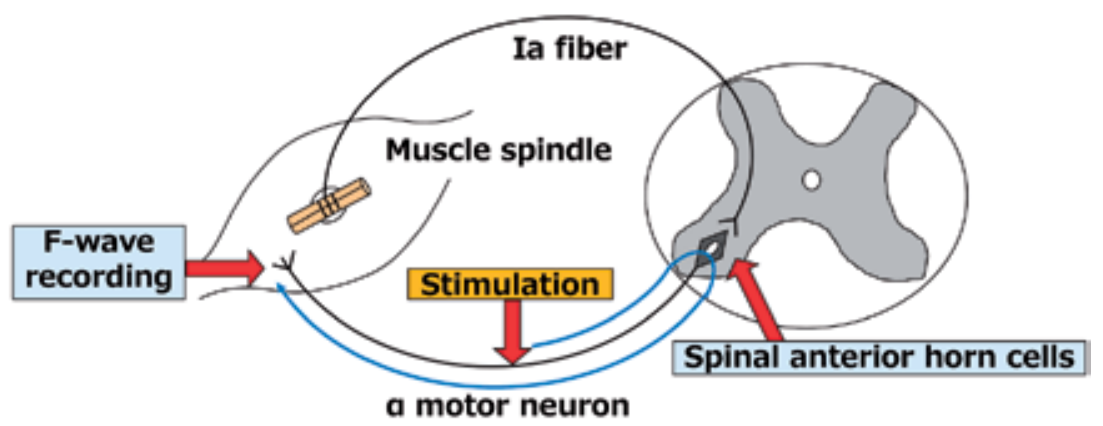

Figure 1.

Mechanism of the F-wave.

\subsection{Materials and methods}

\subsubsection{Participants}

Firstly, we assessed the excitability of spinal motor neurons during MI at 10, 30, 50 , and $70 \% \mathrm{MVC}$ for 10 healthy adults (mean age $=28.7 \pm 4.5$ years). Secondly, we assessed the excitability of spinal motor neurons during MI at 50 and $100 \%$ MVC for 15 healthy adults (mean age $=25.3 \pm 5.0$ years). Written informed consent was obtained prior to participation. The study was approved by the Research Ethics Committee at Kansai University of Health Sciences and conducted in accordance with the Declaration of Helsinki.

\subsubsection{Apparatus and condition for the F-wave recording}

A Viking Quest Electromyography (EMG) machine version 9.0 (Natus Medical, Inc., Pleasanton, CA, USA) was used to record the F-wave. Participants were in supine posture on a bed and instructed to fix one's eyes on the display of a pinch meter (Digital indicator F304A, Unipulse Corp., Japan) (Figure 2).
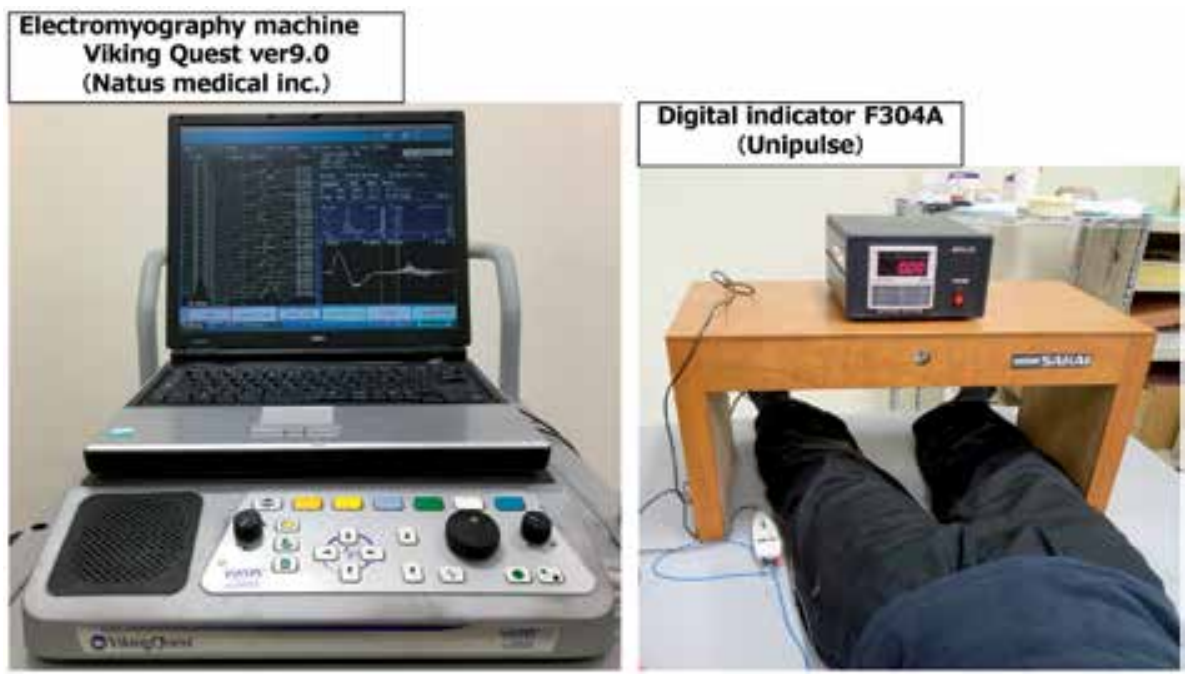

Figure 2.

$F$-wave recording status. 


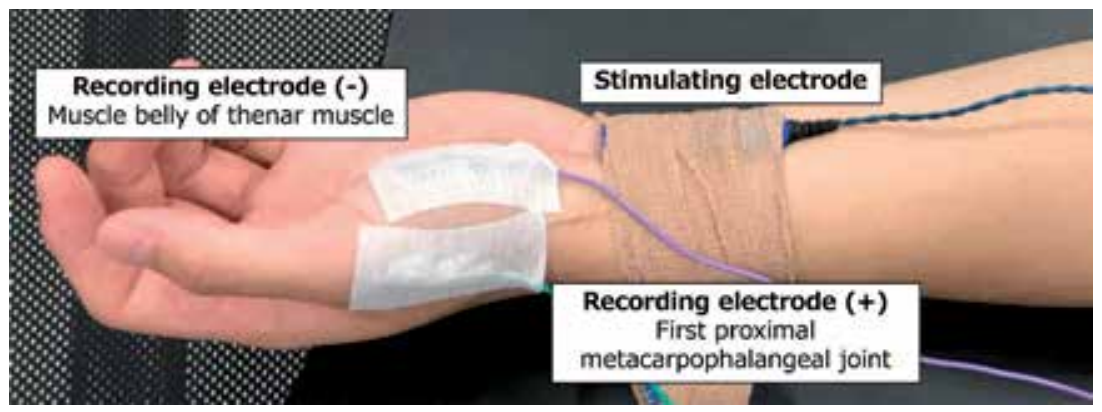

Figure 3.

F-wave recording condition.

The skin impedance was cleaned with an abrasive gel (Nuprep ${ }^{\circledR}$ Skin Prep Gel; Weaver and Company, Inc., Aurora, CO, USA) to maintain below $5 \mathrm{k} \Omega$. The room temperature was maintained at $25^{\circ} \mathrm{C}$. A pair of silver EEG cup electrodes (10mm diameter; Natus Medical, Inc., Pleasanton, CA, USA) was attached over the thenar muscles and the base of the first dorsal metacarpal bone (Figure 3).

The F-wave was evoked from the left thenar muscles by delivering supramaximal electrical stimuli to the left median nerve at wrist. Supramaximal stimulus intensity was determined to be $20 \%$ higher than the maximal stimulus intensity that could elicit the largest $\mathrm{M}$-wave amplitude. Thirty electrical stimuli in each trial were delivered at a duration of $0.2 \mathrm{~ms}$ and frequency of $0.5 \mathrm{~Hz}$. The sensitivity for the F-wave was set at $200 \mu \mathrm{V}$ per division and a sweep of $5 \mathrm{~ms}$ per division. The bandwidth filter ranged from $20 \mathrm{~Hz}$ to $3 \mathrm{kHz}$.

\subsubsection{Experimental protocol}

Firstly, to determine baseline of the excitability of spinal motor neurons, the F-wave was recorded during relaxation for $1 \mathrm{~min}$ (rest). After baseline of the F-wave recording, participants were instructed to learn isometric left thenar muscle activity at 50\% MVC for 1 min with visual feedback. Specifically, participants press the sensor of a pinch meter by the left thumb and index finger at 50\% MVC and keep the 50\% MVC value numerically recorded on the display. For the MI trial, participants imagined isometric left thenar muscle activity at 50\% MVC for $1 \mathrm{~min}$ (50\% MI). After MI trial, the F-wave was recorded during relaxation for $1 \mathrm{~min}$ (post). This protocol was repeated for 10, 30, 70, and 100\% MI conditions. Each condition was performed randomly on different days.

\subsection{Data analysis for the F-wave}

The F-wave data in each trial were analyzed with respect to two parameters: persistence and the F/M amplitude ratio. The minimum peak-to-peak amplitude of F-waves was $20 \mu \mathrm{V}$ [17, 32]. Persistence was defined as the number of detected F-wave responses to 30 electrical stimuli and expressed as percentage (\%).

Persistence reflects the number of backfiring spinal anterior horn cells $[28,29]$. The F/M amplitude ratio was defined as the mean amplitude of all detected F-wave responses divided by the $\mathrm{M}$-wave amplitude and expressed as percentage (\%). The F/M amplitude ratio reflects the size, number, and synchronization of backfiring spinal anterior horn cells $[29,33]$. Therefore, persistence and the F/M amplitude ratio indicate the excitability of spinal motor neurons. 


\subsection{Statistical analysis}

\subsubsection{The F-wave during 10-70\% MI condition}

IBM SPSS statistics version 26 (IBM Corp., Armonk, NY, USA) was used for statistical analysis. A nonparametric method was used for statistical analysis because the normality of obtained data was not confirmed by the Shapiro-Wilk test.

Persistence and the F/M amplitude ratio among three trials (rest, MI, and post) under each MI condition (10, 30, 50, and 70\%) were compared using the Friedman test and Scheffe's post hoc test.

We also calculated the relative value obtained by dividing the F-wave data during MI under four MI conditions by that at rest. The relative values among four MI conditions were compared using the Friedman test. The threshold for statistical significance was set at $\mathrm{p}=0.05$.

\subsubsection{The F-wave during 50 and 100\% MI condition}

Persistence and the F/M amplitude ratio among three trials (rest, MI, and post) under each MVC MI condition were compared using the Friedman test and Scheffe's post hoc test. The relative values between two MI conditions were compared using the Wilcoxon signed rank test.

\subsection{Results}

\subsubsection{The F-wave during 10-70\% MI condition}

Persistence during MI under all MI conditions was significantly higher than the rest (Tables 1-4). The F/M amplitude ratio during MI under 10, 30, and 50\% MI conditions was significantly higher than rest (Tables 1-3). The F/M amplitude ratio during MI under 70\% MI condition was tended to be increased than rest (Table 4).

The relative values of persistence and the F/M amplitude ratio did not demonstrate significant differences among all MI conditions (Table 5).

\subsubsection{The F-wave during 50 and 100\% MI condition}

Persistence during MI under the two MI conditions was significantly higher than rest (Tables 6, 7). The F/M amplitude ratio during MI under the two MI conditions was significantly higher than rest (Tables 6 and 7).

\begin{tabular}{lccc} 
& rest & $10 \%$ MI & post \\
\hline Persistence (\%) & $61.8 \pm 12.6$ & $91.9 \pm 9.70^{* *}$ & $73.1 \pm 20.7$ \\
F/M amplitude ratio (\%) & $0.90 \pm 0.35$ & $2.46 \pm 2.61 * *$ & $1.18 \pm 0.67$ \\
\hline Mean \pm SD. & & \\
$* * p<0.01 ;$ significant difference between rest and 10\% MI trial. & \\
$10 \%$ MI: Motor imagery of isometric thenar muscle activity at 10\% MVC
\end{tabular}

Table 1.

The F-wave under $10 \%$ MI condition. 


\begin{tabular}{lccc}
\hline & rest & $30 \%$ MI & post \\
\hline Perslstence (\%) & $61.2 \pm 19.5$ & $88.0 \pm 12.2 *$ & $60.0 \pm 18.7$ \\
F/M amplitude ratio (\%) & $1.00 \pm 0.94$ & $2.92 \pm 2.95 *$ & $1.11 \pm 0.52$ \\
Mean \pm SD. & & \\
* $p<0.05$; significant difference between rest and 30\% MI trial. & \\
$30 \%$ MI: Motor imagery of isometric thenar muscle activity at 30\% MVC
\end{tabular}

Table 2.

The F-wave under 30\% MI condition.

\begin{tabular}{lccc}
\hline & rest & $50 \%$ MI & post \\
\hline Persistence (\%) & $62.7 \pm 22.3$ & $94.0 \pm 9.40^{*}$ & $65.5 \pm 27.0$ \\
F/M amplitude ratio (\%) & $1.08 \pm 0.28$ & $2.60 \pm 2.30^{* * *}$ & $0.98 \pm 0.40$
\end{tabular}

Mean \pm SD.

$* \rho<0.05$; significant difference between rest and 50\% MI trial.

${ }^{*} p<0.01$; significant difference between rest and $50 \% \mathrm{MI}$ trial.

$50 \%$ MI: Motor imagery of isometric thenar muscle activity at $50 \%$ MVC

Table 3 .

The F-wave under 50\% MI condition.

\begin{tabular}{lccc}
\hline & rest & $70 \% \mathrm{MI}$ & post \\
\hline Persistence (\%) & $\mathbf{5 5 . 9} \pm \mathbf{1 7 . 6}$ & $\mathbf{8 8 . 1} \pm 10.8 * *$ & $65.3 \pm 19.9$ \\
F/M amplitude ratio (\%) & $0.94 \pm 0.33$ & $1.79 \pm 1.23$ & $1.11 \pm 0.44$ \\
\hline
\end{tabular}

Mean \pm SD.

$* * p<0.01$; slgniflcant difference between rest and $70 \%$ MI trlal.

$70 \%$ MI: Motor imagery of isometric thenar muscle activity at $70 \%$ MVC

Table 4.

The F-wave under $70 \%$ MI condition.

\begin{tabular}{lcccc} 
& $\begin{array}{c}10 \% \mathrm{MI} \\
\text { condition }\end{array}$ & $\begin{array}{c}30 \% \mathrm{MI} \\
\text { condition }\end{array}$ & $\begin{array}{c}50 \% \mathrm{MI} \\
\text { condition }\end{array}$ & $\begin{array}{c}70 \% \mathrm{MI} \\
\text { condition }\end{array}$ \\
\hline $\begin{array}{l}\text { relative values of } \\
\text { persistence }\end{array}$ & $1.53 \pm 0.31$ & $1.58 \pm 0.61$ & $1.78 \pm 0.93$ & $1.69 \pm 0.45$ \\
$\begin{array}{l}\text { relative values of F/M } \\
\text { amplitude ratio }\end{array}$ & $2.40 \pm 1.38$ & $3.31 \pm 0.56$ & $2.52 \pm 1.96$ & $2.10 \pm 1.37$ \\
$\begin{array}{l}\text { Mean } \pm \text { SD. } \\
\begin{array}{l}\text { MI: Mator imagery of isometric thenar } \\
\text { muscle activity }\end{array}\end{array}$ & & & \\
\hline
\end{tabular}

Table 5.

Comparison of relative values of the F-wave among $10 \% \mathrm{MI}, 30 \% \mathrm{MI}, 50 \% \mathrm{MI}$, and $70 \% \mathrm{MI}$. 


\begin{tabular}{lccc} 
& rest & $50 \% \mathrm{MI}$ & post \\
\hline Persistence (\%) & $50.8 \pm 21.7$ & $88.2 \pm 13.2 * *$ & $\mathbf{4 8 . 3} \pm 19.9$ \\
F/M amplitude ratio (\%) & $1.71 \pm 0.89$ & $3.96 \pm 4.56 * *$ & $1.29 \pm 0.56$ \\
Mean \pm SD. & & & \\
$-* p<0.01$; significant difference between rest and $50 \% \mathrm{MI}$ trial. & \\
\hline
\end{tabular}

Table 6.

The persistence and F/M amplitude ratio under 50\% MI condition.

\begin{tabular}{lccc} 
& rest & $100 \% \mathrm{MI}$ & post \\
\hline Persistence $(\%)$ & $60.8 \pm 24.9$ & $91.9 \pm 7.58^{* *}$ & $60.7 \pm 21.5$ \\
F/M amplitude ratio (\%) & $1.32 \pm 1.12$ & $3.57 \pm 4.67^{* * *}$ & $1.39 \pm 1.25$ \\
Mean \pm SD. & & \\
$* * p<0.01$; significant difference between rest and $100 \%$ MI trial. & \\
\hline
\end{tabular}

Table 7.

The persistence and F/M amplitude ratio under $100 \%$ MI condition.

The relative values of the persistence and $\mathrm{F} / \mathrm{M}$ amplitude ratio did not demonstrate significant differences between two MI conditions (Table 8).

\subsection{Discussion}

Both persistence and the F/M amplitude ratio significantly increased during MI under all five MI conditions. Previous studies demonstrated that various brain regions, including the primary motor cortex, premotor area, supplementary motor area, prefrontal cortex, parietal lobule, cingulate area, cerebellum, and basal ganglia, contribute to motor preparation and planning during MI $[34,35]$. Therefore, it is considered that activation of the central nervous system contributing to motor preparation and planning during $\mathrm{MI}$ is responsible for increased excitability of the spinal motor neurons via the descending pathways, such as the corticospinal and extrapyramidal tracts.

\begin{tabular}{lcc} 
& $50 \%$ MI condition & 100\% MI condition \\
\hline relative values of persistence & $2.04 \pm 1.17$ & $2.06 \pm 1.71$ \\
relative values of F/M amplitude ratio & $2.75 \pm 2.04$ & $2.53 \pm 1.76$ \\
\hline
\end{tabular}

Mean \pm SD.

MI: Motor imagery of isometric thenar muscle activity

Table 8.

Comparison of relative values of the F-wave between $50 \%$ MI and 100\% MI condition. 
Furthermore, participants performed MI while holding the sensor of a pinch meter. Mizuguchi et al. [36] reported that while holding an object, the corticospinal excitability during MI was modulated by a combination of tactile and proprioceptive inputs. Thus, it is plausible that holding the pinch meter sensor during MI caused tactile and proprioceptive perceptions to cooperatively increase the excitability of the spinal motor neurons along the MI-activated pathways.

Relative values of persistence and the $\mathrm{F} / \mathrm{M}$ amplitude were similar among all $\mathrm{MI}$ conditions. This result indicated that the magnitude of imagined muscle contraction strength may not affect the excitability of the spinal motor neurons. Bonnet et al. [37] reported that the H-reflex amplitude during MI was similar between 2 and 10\% MI conditions. Hale et al. [38] also reported that the H-reflex amplitude during MI of ankle plantar flexion was similar among five (i.e., 20, 40, 60, 80, and 100\% MVC) MI conditions. Similarly, Aoyama et al. [39] reported that the H-reflex amplitude during MI was similar between 50 and 100\% MI conditions. MI is the mental representation of a movement in the absence of any overt movement [9]. The neural mechanism that inhibits actual movement and muscle contraction during MI may be involved in this outcome. Park et al. [40] reported that MEP amplitudes during MI were similar among all six (i.e., 10, 20, 30, 40, 50, and 60\% MVC) MI conditions. Furthermore, magnitude of the primary motor cortex activity during MI did not correlate with the imagined muscle contraction strength, whereas activities of the supplementary motor and premotor areas during MI strongly correlated with it [41]. The supplementary motor and premotor areas have crucial roles in larger force generation [42], motor planning, preparation, and inhibition $[43,44]$. Thus, these areas may inhibit actual muscle contractions depending on the magnitude of the muscle contraction strength. These areas are also connected directly to the primary motor cortex, and inhibitory inputs from them may suppress any additional primary motor cortex excitation conferred by MI with a high imagined contraction strength. Therefore, the degree of excitability of spinal motor neurons during MI at various imagined muscle contraction strengths may be modulated by both excitatory and inhibitory inputs from the central nervous system.

\subsection{Conclusion}

The result of these previous studies demonstrated that MI can increase the excitability of spinal motor neurons. Further, MI at slight imagined muscle contraction strength (i.e., 10\% MVC) can substantially facilitate the excitability of spinal motor neurons.

\section{The influence of duration of MI on the excitability of spinal motor neurons}

\subsection{Background and purpose}

In our previous studies, duration of MI was 1 min [23-26]. However, Driskell et al. [45] indicated that 10-15 min may be appropriate for duration of MI training session. Further, Twinning et al. [46] indicated that $5 \mathrm{~min}$ is the temporal limit to concentrate and perform MI. Therefore, we aimed to investigate the influence of duration of MI on the excitability of spinal motor neurons $[25,26,47]$. 


\subsection{Materials and methods}

\subsubsection{Participants}

Eleven healthy adults participated (mean age $=26.4 \pm 6.0$ years). Written informed consent was obtained prior to participation. The study was approved by the Research Ethics Committee at Kansai University of Health Sciences and conducted in accordance with the Declaration of Helsinki.

\subsubsection{Experimental protocol}

Firstly, to determine the baseline of the excitability of spinal motor neurons, the F-wave was recorded during relaxation for $1 \mathrm{~min}$ (rest). Subsequently, participants were instructed to learn isometric left thenar muscle activity at 50\% MVC for $1 \mathrm{~min}$. For MI trial, participants imagined isometric left thenar muscle activity at $50 \% \mathrm{MVC}$ for $1 \mathrm{~min}$. The $\mathrm{F}$-wave was recorded at 1,3 , and $5 \mathrm{~min}$ after beginning of MI (1-, 3-, and 5-min MI). Immediately after MI trial for $5 \mathrm{~min}$, the F-wave was recorded during relaxation for $1 \mathrm{~min}$ (post).

After F-wave recording, participants evaluated their vividness of MI (i.e., how vividly they could imagine isometric thenar muscle activity at 50\% MVC) at 1-, 3-, and 5-min MI using a seven-point Likert scale ranging from 1 (very difficult to perform MI vividly) to 7 (very easy to perform MI vividly).

\subsection{Statistical analysis}

A nonparametric method was used for statistical analyses because the normality of obtained data was not confirmed with the Shapiro-Wilk test. Persistence and the F/M amplitude ratio among five trials (rest, 1-, 3-, and 5-min MI, and post) were compared using the Friedman test and Scheffe's post hoc test. Rating scores of MI vividness at 1-, 3-, and 5-min MI were compared using the Friedman test and Scheffe's post hoc test. The threshold for statistical significance was set at $p=0.05$.

\subsection{Results}

Persistence at 1- and 3-min MI trial was significantly higher than rest (Table 9). No significant difference in persistence was observed between 5-min MI and rest trial (Table 9). Further, no significant differences in persistence were confirmed among 1-, 3-, and 5-min MI trial.

\begin{tabular}{|c|c|c|c|c|c|}
\hline & rest & 1-min MI & 3-min MI & 5-min MI & post \\
\hline Persistence (\%) & $53.5 \pm 26.8$ & $88.4 \pm 13.3$ & $85.5 \pm 14.8$ & $83.7 \pm 17.3$ & $54.0 \pm 25.5$ \\
\hline $\begin{array}{l}\text { F/M amplitude ratio } \\
(\%)\end{array}$ & $0.91 \pm 0.38$ & $2.00 \pm 0.86^{k+}$ & $1.84 \pm 1.00 *$ & $1.70 \pm 0.71^{+}$ & $0.97 \pm 0.39$ \\
\hline \multicolumn{6}{|l|}{ Mean \pm SD. } \\
\hline
\end{tabular}

Table 9.

The F-wave during MI for 5 min. 


\section{1-mIn MI $\quad 3-\mathrm{mln}$ MI $\quad 5-\mathrm{mln} M I$}

\begin{tabular}{|c|c|c|c|}
\hline MI vividness & $5.71 \pm 0.76$ & $3.57 \pm 0.53$ & $2.29 \pm 0.76^{*}$ \\
\hline \multicolumn{4}{|l|}{ Mean \pm SD. } \\
\hline
\end{tabular}

Table 10.

Rating scores of MI vividness during MI for 5 min.

The F/M amplitude ratio at 1- and 3-min MI trial was significantly higher than rest (Table 9). The F/M amplitude ratio at 5-min $\mathrm{MI}$ trial was significantly smaller than 1- and 3-min MI trial (Table 9). Further, no significant difference in the F/M amplitude ratio was observed between 5-min MI and rest trial (Table 9).

The rating score of MI vividness at 5-min MI trial was significantly smaller that 1-min MI trial (Table 10).

\subsection{Discussion}

The results for the F-wave indicated that duration of MI for 1-3 min positively affects the excitability of spinal motor neurons. Further, the results of vividness of $\mathrm{MI}$ indicated that temporal limitation of MI, participants can perform MI vividly, may be $3 \mathrm{~min}$.

It is considered that mental fatigue and/or habituation were involved in this result. Repetitive MI of a handgrip movement decreases the corticospinal excitability [48]. Rozand et al. [49] also demonstrated that participants felt difficulty to maintain their focus on imagined movement due to mental fatigue. Therefore, mental fatigue caused by sustained mental activity may affect the excitability of spinal motor neurons.

Brain activity was decreased by habituation after cognitive motor task for $10 \mathrm{~min}$ [50]. Further, the corticospinal excitability was also decreased by habituation [50]. At spinal level, the T-reflex amplitude was decreased after sustained mental activity for $20 \mathrm{~min}$ [51]. Therefore, habituation after sustained mental activity as MI can alter the corticospinal excitability, including the excitability of spinal motor neurons.

\subsection{Conclusion}

The result of this study demonstrated that longer duration of MI above $3 \mathrm{~min}$ has no facilitatory effect on the excitability of spinal motor neurons. Therefore, in physical therapy, the duration of MI should be considered.

\section{The influence of imagery strategy on the excitability of spinal motor neurons}

\subsection{Background and purpose}

Previously, we investigated the influence of MI of isometric thenar muscle activity on the excitability of spinal motor neurons [18, 23-26]. Previous results indicated that the MI of thenar muscle activity at 50\% MVC can increase the excitability of spinal motor neurons. However, there were individual differences in facilitation amount of the excitability of spinal motor neurons. 
MI includes various components of perception that can be associated with actual movement [52]. Therefore, the effects of MI may differ depending on the choice of sensory modality. Then, we investigated that imagery strategy on the excitability of spinal motor neurons [53].

\subsection{Materials and methods}

\subsubsection{Participants}

Fourteen healthy adults (mean age $=23.4 \pm 4.8$ years) participated. Written informed consent was obtained prior to participation. The study was approved by the Research Ethics Committee at Kansai University of Health Sciences and conducted in accordance with the Declaration of Helsinki.

\subsubsection{Experimental protocol}

For the rest trial (rest), to determine the baseline excitability of the spinal motor neurons, the F-wave was recorded during relaxation for $1 \mathrm{~min}$. Subsequently, participants were instructed to exert isometric left thenar muscle contraction at $50 \%$ MVC for 1 min with visual feedback. Simultaneously, participants learned two imagery strategies: somatosensory (tactile and pressure perception of thumb finger pulp during pressing the sensor of pinch meter) and kinesthetic (thenar muscle contraction during pressing the sensor of pinch meter at 50\% MVC). Subsequently, participants performed the somatosensory imagery (SI), kinesthetic imagery (KI), and combined somatosensory and kinesthetic imagery (SKI). In SKI trial, participants imagined somatosensory and kinesthetic sensation simultaneously. The duration of each MI session was $1 \mathrm{~min}$.

After the F-wave recording, participants evaluated difficulty of each imagery strategy by using a five-point Likert scale, ranging from 1 (very hard to image vividly) to 5 (very easy to image vividly).

\subsection{Statistical analysis}

A nonparametric method was used for statistical analyses because the normality of obtained data was not confirmed with the Shapiro-Wilk test. Persistence and the F/M amplitude ratio among four trials (rest, SI, KI, and SKI) were compared using the Friedman test and Scheffe's post hoc test. Rating scores of each imagery strategy (SI, KI, and SKI) were compared using the Friedman test and Scheffe's post hoc test. The threshold for statistical significance was set at $p=0.05$.

\subsection{Results}

Persistence was significantly higher during SI and KI trials than at rest (Table 11). Persistence tended to be higher during SKI than at rest (Table 11).

The F/M amplitude ratio was significantly higher during $\mathrm{KI}$ than at rest (Table 11).

The rating score of SKI vividness was significantly smaller than rest (Table 12).

\subsection{Discussion}

Persistence and the F/M amplitude ratio were significantly increased during KI. Described in Introduction section, numerous studies demonstrated that various brain areas, including the primary motor cortex, were activated during $\mathrm{KI}[34,35]$. 


\begin{tabular}{lcccc}
\hline & rest & SI & KI & SKI \\
\hline Persistence (\%) & $53.7 \pm 21.9$ & $73.4 \pm 19.6 * *$ & $74.3 \pm 23.7^{+*}$ & $68.4 \pm 21.8$ \\
F/M amplitude ratio & $0.83 \pm 0.32$ & $1.20 \pm 0.50$ & $1.33 \pm 0.70^{*}$ & $1.28 \pm 0.65$ \\
$(\%)$ & & & & \\
Mean \pm SD. & & & \\
${ }^{+} p<0.01$; significant difference between rest and KI trial. \\
${ }^{+*} p<0.01$; signiflcant difference between rest and SI trlal. \\
${ }^{*} p<0.05$; significant difference between rest and KI trial.
\end{tabular}

Table 11.

The F-wave during SI, KI, and SKI, trail.

\begin{tabular}{lccc} 
& SI & KI & SKI \\
\hline MI vividness & $3.64 \pm 0.63$ & $3.86 \pm 0.95$ & $3.21 \pm 0.89 *$ \\
Mean \pm SD. & \\
${ }^{8}$ < 0.05 ; significant difference between $\mathrm{KI}$ and SKI trial &
\end{tabular}

SI, somatosensory Imagery; KI, klnesthetlc Imagery; SKI, comblned somatosensory and kinesthetic imagery

Table 12.

Rating scores of MI vividness during SI, KI, and SKI, trail.

The corticospinal excitability was significantly increased during KI [2, 15]. Therefore, the excitability of spinal motor neurons during KI may be increased via the descending pathways, such as the corticospinal and extrapyramidal tracts.

Although there was no significant difference in the $\mathrm{F} / \mathrm{M}$ amplitude ratio between SI and rest trial, persistence was significantly increased during SI than rest. We did not expect this result previously, because there are no previous studies reported that SI increases the corticospinal excitability including that of the primary motor cortex. One possible is that SI adopted in this study included kinesthetic components. Participants in this study imagined tactile and pressure perception accompanied with holding the sensor of a pinch meter. Therefore, it is plausible that participants imagined tactile and perception along with thenar muscle activity.

Persistence during SKI was tended to be higher than rest, and the rating score of SKI vividness was the lowest among all imagery strategies (SI, KI, and SKI). Participants in this study were instructed to pay attention to kinesthetic and somatosensory perception simultaneously. The decline in the amount of attention that can be allocated to each imagery strategy may have increased difficulty for participants to perform SKI vividly. Indeed, Williams et al. [54] indicated that there are positive correlation between the corticospinal excitability and MI vividness.

\subsection{Conclusion}

The result of this study indicated that KI may have a greater effect on the excitability of spinal motor neurons.

\section{How to use MI in neurorehabilitation}

In post-stroke and spinal cord injury, motor cortex excitability was decreased due to damage of neural substrates, loss of sensory inputs, and disuse of affected 
limb [1]. Further, the MEP amplitude was decreased after stroke [55] and spinal cord injury [56]. Additionally, a significant reduction of spinal motor neuron excitability has been shown in the post-stroke acute phase [4] and spinal cord injury [57]. Facilitating the corticospinal excitability is closely related to functional motor recovery [58]. Therefore, the immediate enrollment in rehabilitation programs aiming to facilitate the corticospinal excitability, including the excitability of spinal motor neurons, should be important. Our previous researches investigated the excitability of spinal motor neurons during MI in only healthy volunteers. However, Cicinelli et al. [59] reported that the MEP amplitude was significantly facilitated during MI in post-stroke. Naseri et al. [60] reported that the amplitude and persistence of the F-wave were significantly increased during MI in post-stroke. Further, similar effect was observed in spinal cord injury [61]. Also, in Parkinson's disease, the amplitude and persistence of the F-wave were significantly increased during MI [62]. Therefore, MI has a facilitating effect on the corticospinal excitability, including the excitability of spinal motor neurons, for central nervous system disorder.

Next, about definite method of MI, our research group revealed that MI of isometric thenar muscle activity at 50\% MVC can increase the excitability of spinal motor neurons. Additionally, imagined muscle contraction strength did not affect facilitation amount of the excitability of spinal motor neurons. In physical therapy for facilitating the excitability of spinal motor neurons, slight (i.e., 10\% MVC) imagined muscle contraction strength may be sufficient. Then, kinesthetic imagery could more facilitate the excitability of spinal motor neurons than somatosensory imagery. Stinear et al. [63] reported that kinesthetic imagery can significantly increase the corticospinal excitability. Therefore, to facilitate the excitability of spinal motor neurons, kinesthetic perception may be used for imagery strategy. Also considering mental fatigue and habituation, duration of MI may be less than 3 min.

\title{
Conflict of interest
}

The authors declare no conflict of interest.

\section{Author details}

\author{
Yoshibumi Bunno \\ Graduate School of Health Sciences, Graduate School of Kansai University of \\ Health Sciences, Osaka, Japan
}

*Address all correspondence to: bunno@kansai.ac.jp

\section{IntechOpen}

(C) 2020 The Author(s). Licensee IntechOpen. This chapter is distributed under the terms of the Creative Commons Attribution License (http://creativecommons.org/licenses/ by/3.0), which permits unrestricted use, distribution, and reproduction in any medium, provided the original work is properly cited. (cc) BY 


\section{References}

[1] Liepert J, Bauder H, Wolfgang HR, Miltner WH, Taub E, Weiller C.

Treatment-induced cortical reorganization after stroke in humans. Stroke. 2000;31:1210-1216. DOI: 10.1161/01.str.31.6.1210

[2] Foltys H, Krings T, Meister IG, Sparing R, Boroojerdi B, Thron A, et al. Motor representation in patients rapidly recovering after stroke: $A$ functional magnetic resonance imaging and transcranial magnetic stimulation study. Clinical Neurophysiology. 2003;114:2404-2415. DOI: 10.1016/ S1388-2457(03) 00263-3

[3] Fierro B, Raimondo D, Modica A. Analysis of $\mathrm{F}$ response in upper motoneurone lesions. Acta Neurologica Scandinavica. 1990;82:329-334. DOI: 10.1111/j.1600-0404.1990.tb03311.x

[4] Drory VE, Neufeld MY, Korczyn AD. F-wave characteristics following acute and chronic upper motor neuron lesions. Electromyography and Clinical Neurophysiology. 1993;33:441-446

[5] Nakayama H, Jørgensen HS, Raaschou HO, Olsen TS. Recovery of upper extremity function in stroke patients: The Copenhagen stroke study. Archives of Physical Medicine and Rehabilitation. 1994;75:394-398. DOI: 10.1016/0003-9993(94)90108-2

[6] Kreisei SH, Hennerici MG, Bäzner H. Pathophysiology of stroke rehabilitation: The natural course of clinical recovery, use-dependent plasticity and rehabilitative outcome. Cerebrovascular Diseases. 2007;23:243255. DOI: $10.1159 / 000098323$

[7] Horn SD, DeJong G, Smout RJ, Gassaway J, James R, Conroy B. Stroke rehabilitation patients, practice, and outcomes: Is earlier and more aggressive therapy better? Archives of Physical Medicine and Rehabilitation.
2005;86:S101-S114. DOI: 10.1016/j. apmr.2005.09.016

[8] Coleman ER, Moudgal R, Lang K, Hyacinth HI, Awosika OO, Kissela BM, et al. Early rehabilitation after stroke: A narrative review. Current Atherosclerosis Reports. 2017;19:59. DOI: 10.1007/ s11883-017-0686-6

[9] Guillot A, Di Rienzo F, Maclntyre T, Moran A, Collet C. Imagining is not doing but involves specific motor commands: A review of experimental data related to motor inhibition. Frontiers in Human Neuroscience. 2012;6:247. DOI: 10.3389/ fnhum.2012.00247

[10] Lotze M, Montoya P, Erb M, Hülsmann E, Flor H, Klose U, et al. Activation of cortical and cerebellar motor areas during executed and imagined hand movements: An fMRI study. Journal of Cognitive Neuroscience. 1999;11:491-501. DOI: 10.1162/089892999563553

[11] Luft AR, Skalej M, Stefanou A, Klose U, Voigt K. Comparing motionand imagery-related activation in the human cerebellum: A functional MRI study. Human Brain Mapping. 1998;6:105-113. DOI: 10.1002/(sici)10970193(1998)6:2<105::aid-hbm3>3.0.co;2-7

[12] Stephan KM, Fink GR, Passingham RE, Silbersweig D, Ceballos-Baumann AO, Frith CD, et al. Functional anatomy of the mental representation of upper extremity movements in healthy subjects. Journal of Neurophysiology. 1995;73:373-386. DOI: 10.1152/jn.1995.73.1.373

[13] Nakano H, Ueta K, Osumi M, Morioka S. Brain activity during the observation, imagery, and execution of tool use: An fNIRS/EEG study. Journal of Novel Physiotherapies. 2012;S1:009. DOI: 10.4172/2165-7025.S1-009 
[14] Kasai T, Kawai S, Kawanishi M, Yahagi S. Evidence for facilitation of motor evoked potentials (MEPs) induced by motor imagery. Brain Research. 1997;744:147-150. DOI: 10.1016/S0006-8993(96)01101-8

[15] Stinear CM, Byblow WD. Modulation of corticospinal excitability and intracortical inhibition during motor imagery is task-dependent. Experimental Brain Research. 2004;157:351-358. DOI: 10.1007/ s00221-004-1851-z

[16] Taniguchi S, Kimura J, Yamada T, Ichikawa H, Hara M, Fujisawa R, et al. Effect of motion imagery to counter rest-induced suppression of $\mathrm{F}$-wave as a measure of anterior horn cell excitability. Clinical Neurophysiology. 2008;119:1346-1352. DOI: 10.1016/j. clinph.2007.11.179

[17] Hara M, Kimura J, Walker DD, Taniguchi S, Ichikawa H, Fujisawa R, et al. Effect of motor imagery and voluntary muscle contraction of the F-wave. Muscle \& Nerve. 2010;42:208212. DOI: $10.1002 /$ mus. 21667

[18] Suzuki T, Bunno Y, Onigata C, Tani M, Uragami S. Excitability of spinal neural function during several motor imagery tasks involving isometric opponens pollicis activity. NeuroRehabilitation. 2013;33:171-176. DOI: 10.3233/NRE-130942

[19] Hashimoto R, Rothwell JC. Dynamic changes in corticospinal excitability during motor imagery. Experimental Brain Research. 1999;125:75-81. DOI: $10.1007 / \mathrm{s} 002210050660$

[20] Stinear CM, Fleming MK, Byblow WD. Lateralization of unimanual and bimanual motor imagery. Brain Research. 2006;1095:139147. DOI: 10.1016/j.brainres.2006.04.008

[21] Oishi K, Kimura M, Yasukawa M, Yoneda T, Maeshima T. Amplitude reduction of H-reflex during mental movement simulation in elite athletes. Behavioural Brain Research. 1994;62:5561. DOI: 10.1016/0166-4328(94)90037-X

[22] Suzuki T, Fujiwara T, Takeda I. Excitability of the spinal motor neuron pool and $\mathrm{F}$-waves during isometric ipsilateral and contralateral contraction. Physiotherapy Theory and Practice. 1993;9:19-24. DOI: 10.3109/09593989309036482

[23] Bunno Y, Onigata C, Suzuki T. The imagined muscle contraction strengths did not affect the changes of spinal motor neurons excitability. Journal of Novel Physiotherapies. 2016;S3:008. DOI: 10.4172/2165-7025.S3-008

[24] Bunno Y, Fukumoto Y, Todo M, Onigata C. The effect of motor imagery on spinal motor neuron excitability and its clinical use in physical therapy. In: Suzuki T, editor. Neurological Physical Therapy. United Kingdom: IntechOpen; 2017. pp. 29-50. DOI: $10.5772 / 67471$

[25] Bunno Y. The application of motor imagery to neurorehabilitation. In: Larrivee D, editor. Evolving BCI TherapyEngaging Brain State Dynamics. United Kingdom: Intech; 2018. pp. 53-71. DOI: 10.5772/intechopen.75411

[26] Bunno Y. Effectiveness of motor imagery on physical therapy: Neurophysiological aspects of motor imagery. In: Bernardo-Filho M, Sá-Caputo D, Taiar R, editors. Physical Therapy Effectiveness. United Kingdom: Intech; 2019. pp. 1-19. DOI: 10.5772/ intechopen.90277

[27] Kimura J. F-wave velocity in the central segment of the median and ulnar nerves. A study in normal subjects and in patients with Charcot-Marie-tooth disease. Neurology. 1974;24:539-546. DOI: 10.1212/WNL.24.6.539

[28] Mesrati F, Vecchierini MF. F-waves neurophysiology and clinical 
value. Neurophysiologie Clinique. 2004;34:217-243. DOI: $10.1016 / j$. neucli.2004.09.005

[29] Fisher MA. F-waves-physiology and clinical uses. The Scientific World Journal. 2007;7:144-160. DOI: 10.1100/ tsw.2007.49

[30] Mercuri B, Wassemann EM, Manqanotti P, Ikoma K, Samii A, Hallett M. Cortical modulation of spinal excitability: An F-wave study. Electroencephalography and Clinical Neurophysiology. 1996;101:16-24. DOI: 10.1016/0013-4694(95)00164-6

[31] Rossini PM, Rossi S, Pasqualetti P, Tacchio F. Cortical excitability modulation to hand muscles during movement imagery. Cerebral Cortex. 1999;9:161-167. DOI: 10.1093/ cercor/9.2.161

[32] Fisher MA. H reflexes and F waves fundamentals, normal and abnormal patters. Neuroimaging Clinics of North America. 2002;20:339-360. DOI: 10.1016/s0733-8619(01)00004-4

[33] Peioglou-Harmoussi S, Fawcett PR, Howel D, Barwick DD. F-responses: A study of frequency, shape and amplitude characteristics in healthy control subjects. Journal of Neurology, Neurosurgery, and Psychiatry. 1985;48:1159-1164. DOI: 10.1136/jnnp.48.11.1159

[34] Hanakawa T, Dimyan MA, Hallett M. Motor planning, imagery, and execution in the distributed motor network: A time-course study with functional MRI. Cerebral Cortex. 2008;18:2775-2788. DOI: 10.1093/ cercor/bhn036

[35] Hanakawa T. Organizing motor imageries. Neuroscience Research. 2016;104:56-63. DOI: 10.1016/j. neures.2015.11.003

[36] Mizuguchi N, Sakamoto M, Muraoka T, Nakagawa K, Kanazawa S,
Nakata $\mathrm{H}$, et al. The modulation of corticospinal excitability during motor imagery of action with objects. PLoS One. 2011;6:e26006. DOI: 10.1371/ journal.pone.0026006

[37] Bonnet M, Decety J, Jeannerod M, Requina J. Mental simulation of an action modulates the excitability of spinal reflex pathways in man. Cognitive Brain Research. 1997;5:221-228. DOI: 10.1016/S0926-6410(96)00072-9

[38] Hale BS, Raglin JS, Koceja DM. Effect of mental imagery of a motor task on the Hoffmann reflex. Behavioural Brain Research. 2003;142:81-87. DOI: 10.1016/S0166-4328(02)00397-2

[39] Aoyama T, Kaneko F. The effect of motor imagery on gain modulation of the spinal reflex. Brain Research. 2011;1372:41-48. DOI: 10.1016/j. brainres.2010.11.023

[40] Park WH, Li S. No graded responses of finger muscles to TMS during motor imagery of isometric finger forces. Neuroscience Letters. 2011;494:255-259. DOI: 10.1016/j.neulet.2011.03.027

[41] Romero DH, Lacourse MG, Lawrence KE, Schandler S, Cohen MJ. Event-related potentials as a function of movement parameter variations during motor imagery and isometric action. Behavioural Brain Research. 2000;117:83-96. DOI: 10.1016/ S0166-4328(00)00297-7

[42] Oda S, Shibata M, Moritani T. Force-dependent changes in movement-related cortical potentials. Journal of Electromyography and Kinesiology. 1996;6:247-252. DOI: 10.1016/S1050-6411(96)00010-7

[43] Nakata H, Sakamoto K, Ferretti A, Perrucci MG, Gratta CD, Kakigi R, et al. Somato-motor inhibitory processing in humans: An event-related functional MRI study. NeuroImage. 2008;39:1858-1866. DOI: 10.1016/j. neuroimage.2007.10.041 
[44] Watanabe J, Sugiura M, Sato K, Sato Y, Maeda Y, Matsue Y, et al. The human prefrontal and parietal association cortices are involved in NO-GO performances: An event-related fMRI study. NeuroImage. 2002;17:1207-1216. DOI: $10.1006 /$ nimg.2002.1198

[45] Driskell J, Copper C, Moran A. Does mental practice enhance performance? Journal of Applied Phychology. 1994;79:481-492. DOI: 10.1037/0021-9010.79.4.481

[46] Twining WE. Mental practice and physical practice in learning a motor skill. Research Quarterly. 1949;20:432-435

[47] Bunno Y. Does the duration of motor imagery affect the excitability of spinal anterior horn cells? Somatosensory \& Motor Research. 2018;35:223-228. DOI: 10.1080/08990220.2018.1538963

[48] Kluger BM, Palmer C, Shattuck JT, Triggs WJ. Motor evoked potential depression following repetitive central motor initiation. Experimental Brain Research. 2012;216:585-590. DOI: 10.1007/s00221-011-2962-y

[49] Rozand V, Lebon F, Stapley PJ, Papaxanthis C, Lepers R. A prolonged motor imagery session alter imagined and actual movement durations: Potential implications for neurorehabilitation. Behavioural Brain Research. 2016;297:67-75. DOI: 10.1016/j.bbr.2015.09.036

[50] Tana MG, Montin E, Cerutti S, Bianchi AM. Exploring cortical attentional system by using fMRI during a continuous performance test. Computational Intelligence and Neuroscience. 2010;2010:329213. DOI: $10.1155 / 2010 / 329213$

[51] Brunia CH, Zwaga HJ, van Boxtel A. Tendon reflex amplitude with increasing task difficulty. Ergonomics. 1973;16:495499. DOI: $10.1080 / 00140137308924538$
[52] McNorgan C. A meta-analytic review of multisensory imagery identifies the neural correlates of modality-specific and modalitygeneral imagery. Frontiers in Human Neuroscience. 2012;6:285. DOI: 10.3389/ fnhum.2012.00285

[53] Bunno Y. Imagery strategy affects spinal motor neuron excitability -using kinesthetic and somatosensory imagery. Neuroreport. 2019;30:463-467. DOI: 10.1097/WNR.0000000000001218

[54] Williams J, Pearce AJ, Loporto M, Morris T, Holmes PS. The relationship between corticospinal excitability during motor imagery and motor imagery ability. Behavioural Brain Research. 2012;226:369-375. DOI: 10.1016/j.bbr.2011.09.014

[55] Wrigley PJ, Gustin SM, Macey PM, Nash PG, Gandevia SC, Macefield VG, et al. Anatomical changes in human motor cortex and motor pathways following complete thoracic spinal cord injury. Cerebral Cortex. 2009;19: 224-232. DOI: $10.1093 /$ cercor/bhn072

[56] Diehl P, Kliesch U, Dietz V, Curt A. Impaired facilitation of motor evoked potentials in incomplete spinal cord injury. Journal of Neurology. 2006;253:51-57. DOI: 10.1007/ s00415-005-0921-x

[57] Curt A, Keck ME, Dietz V. Clinical value of $\mathrm{F}$-wave recordings in traumatic cervical spinal cord injury. Electroencephalography and Clinical Neurophysiology. 1997;105:189-193. DOI: $10.1016 / \mathrm{s} 0924-980 x(97) 96626-1$

[58] Triggs WJ, Calvanio R, Levine M. Transcranial magnetic stimulation reveals a hemispheric asymmetry correlate of intermanual differences in motor performance. Neurophychologia. 1997;35:1355-1363. DOI: 10.1016/ s0028-3932(97)00077-8

[59] Cicinelli P, Marconi B, Zaccagnini M, Pasqualetti P, Filippi MM, Rossini PM. 
Imagery-induced cortical excitability changes in stroke: A transcranial magnetic stimulation study. Cerebral Cortex. 2006;16:247-253. DOI: 10.1093/ cercor/bhi103

[60] Naseri M, Petramfar P, Ashraf A. Effect of motor imagery on the F-wave parameters in hemiparetic stroke survivors. Annals of Rehabilitation Medicine. 2015;39:401-408. DOI: 10.5535/arm.2015.39.3.401

[61] Cramer SC, Orr EL, Cohen MJ, Lacourse MG. Effects of motor imagery training after chronic, complete spinal cord injury. Experimental Brain Research. 2007;177:33-242. DOI: 10.1007/s00221-006-0662-9

[62] Suzuki T, Bunno Y, Onigata C, Tani M, Uragami S, Yoshida S. Excitability of spinal neural function during motor imagery in Parkinson's disease. Functional Neurology. 2014;29: 263-267

[63] Stinear CM, Byblow WD, Steyvers M, Levin O, Swinnen SP. Kinesthetic, but not visual, motor imagery modulates corticomotor excitability. Experimental Brain Research. 2006;168:157-164. DOI: 10.1007/s00221-005-0078-y 

from $\mathrm{LF} / \mathrm{HF}$

\author{
Yuki Fukumoto
}

\begin{abstract}
Motor imagery can be helpful for the therapeutic approach for the patients who have difficulty in the actual motion. This chapter explains the following important six points for getting high-effective motor imagery based on the neuroscience. First, excitability of spinal motor neuron was increased by motor imagery. However, adding effect on breathing state, both expiration and inspiration phase on the relax respiration, does not influence the excitability of spinal motor neuron. Also, motor imagery increased the excitability of spinal motor neuron and cardiac sympathetic nerve activity. However, vividness of motor imagery was to converge a degree. Motor practice before motor imagery was important. Motor practice was appropriate for $30 \mathrm{~s}$ using intermittent visual feedback, and for doing motor imagery, time enough was 1 min. Adding motor imagery method was a recommended composite for kinesthetic and visual motor imagery. Unfortunately, motor imagery has few effects for the other hand. Therefore, motor imagery should be done on the ipsilateral side from the previous motor practice.
\end{abstract}

Keywords: motor imagery, motor practice, F-wave, LF/HF, breathing state, motor accuracy, pinch task

\title{
1. Introduction
}

The aim of rehabilitation is to improve motor function. Physical therapists distinguish physical limitations and do therapy for patients. The other effective method was doing self-training for the patient oneself [1]. However, self-training may be carefulness or contraindication when it merges heart trouble and respiratory illness. Therefore, we think necessarily self-training without actual motion, and we suggest motor imagery on self-training. Motor imagery especially involves the activation of cognitive processes from working memory [2]. Motor imagery is not limitation time, place, and using special equipment. Combination therapy for actual motion and motor imagery was improvement of upper limb function than only actual motion in post-stroke hemiparesis patients [3]. The only motor imagery case [4] is the comparison of muscular strengths after motor imagery of the little finger maximum voluntary contraction (MVC) abduction movement for 4 weeks 


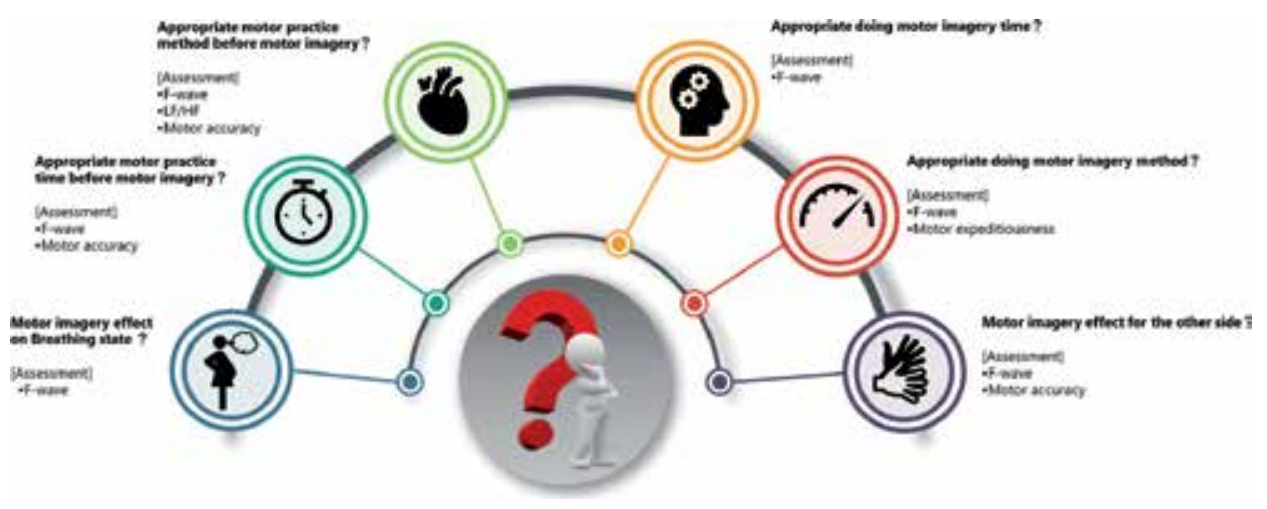

Figure 1.

Important six points for getting high-effective motor imagery.

among motor imagery, physical training, and control groups. They found that muscular strength was reinforced at $30 \%$ in the physical strength training group and at $22 \%$ in the motor imagery group. The reason of increasing muscular strength was to expand the little finger scope at primary motor area. We thought that motor imagery was an effective treatment means. This chapter explains the following important six points for getting high-effective motor imagery (Figure 1).

\section{Motor imagery effect on breathing state?}

\subsection{The difference between expiration and inspiration on spinal cord excitability}

Motor imagery and actual motion have a community of neural system. So, the respiration rates and the heart rate increase during motor imagery [5-7]. A previous study reported that the $\mathrm{F}$-wave was influenced by difference of respiration phase $[8,9]$. We want to be cleared up with the mechanism for the excitability of spinal motor neuron. We thought that the excitability of spinal motor neuron should be caused not secondary to respiration by primarily to the motor imagery.

The average respiration rates are 12-18 every minute on healthy subject. Generally, the ratio of expiration and inspiration is 1.5:1. Therefore, expiration is a little long than inspiration. We were defined to prohibit doing breath holding for making an adjustment to time ratio of expiration and inspiration. But, this role has no problems, because Iwamoto et al. [10] reported that the ratio of expiration and inspiration at 1:1 condition can do respiration on the relax and not voluntarily. In this study, expiration and inspiration every $2 \mathrm{~s}$ are natural. First, F-waves were recorded under these subjects to relax. Next, these subjects were asked to practice pinch force generation at an adjustment of 50\% MVC using visual feedback for $30 \mathrm{~s}$. Finally, F-waves were recorded again under these subjects doing motor imagery.

A Viking Quest electromyography machine [Natus Medical Inc.] was used to record $\mathrm{F}$-waves. We recorded the F-waves by stimulating the left median nerve at the wrist. Supramaximal shocks (adjusted up to the value $20 \%$ higher than the maximal stimulus) were delivered at $0.5 \mathrm{~Hz}$ and $0.2 \mathrm{~ms}$ for $\mathrm{F}$-wave acquisition. We recorded $\mathrm{F}$-waves of the left thenar muscles using a pair of disks attached with collodion to the skin over the eminence of the thumb and the bones of the metacarpophalangeal joint of the thumb. The stimulating electrodes were comprised of a cathode placed over the left median nerve $3 \mathrm{~cm}$ proximal to the palmar crease of the wrist joint and an anode placed $2 \mathrm{~cm}$ more proximally (Figure 2). 


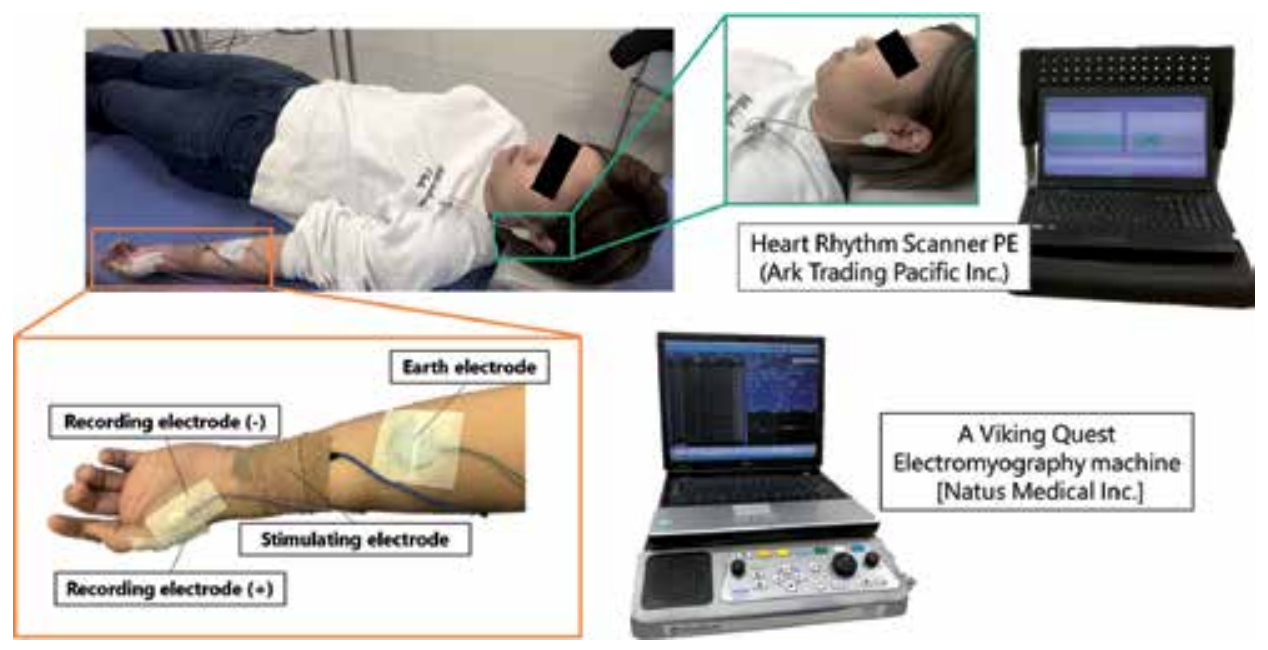

Figure 2.

Recording method for the F-wave.

\begin{tabular}{lcc}
\hline \hline & Expiration & Inspiration \\
\hline Increase rate for F/M amplitude ratio (\%) & $1.0 \pm 1.7$ & $0.7 \pm 1.2$ \\
Increase rate for Persistence (\%) & $12.7 \pm 21.9$ & $8.7 \pm 17.8$ \\
\hline \hline
\end{tabular}

average \pm standard deviation

Table 1.

The result of motor imagery effect on breathing state.

In the result, no significant differences were observed in the increase rate for persistence and F/M amplitude ratio between motor imagery with expiration and inspiration. Respiration have two patterns for the relax or voluntarily. Relax respiration is controlled by brain stem [11]. And, voluntarily respiration is controlled by cerebral cortex. Previous study reported that MEP from hand finger was increased by voluntarily respiration [12]. Hand finger was not related to respiration, but activation of trunk area was influenced for the proximate hand finger area on primary motor area [8]. From the above, the increasing spinal motor neuron might be recognizing only voluntarily respiration. But, this study was only allowed to relax respiration. In conclusion, both expiration and inspiration phase on the relax respiration was not influenced by activation of cerebral cortex (Table 1).

\section{Appropriate motor practice time before motor imagery}

\subsection{Motor imagery effect from motor practice time on the accuracy and spinal cord excitability}

Our previous study [13] investigated motor imagery effect for the motor accuracy on the hand finger. As a result, $30 \mathrm{~s}$ or 1 min motor practice was good to get the high motor imagery effect. But, $10 \mathrm{~s}$ or 2 min motor practice was bad to get the high motor imagery effect. Then, we perceived that the excitability of spinal motor neuron was dependent on motor imagery implementation status.

A total of 44 healthy subjects were randomly and evenly allocated into four groups based on the allowed motor practice time: either 10, 30, 60, or $120 \mathrm{~s}$. 
F-waves were recorded at rest. Next, subjects were asked to practice pinch force generation at $50 \% \mathrm{MVC}$, using visual feedback for guidance. The subjects were then asked to generate pinch force at 50\% MVC without visual feedback. The index of accuracy was recorded. Next, these subjects were asked to perform motor imagery, and F-waves were recorded. Finally, these subjects repeated the non-guided pinch task again.

An index reflecting the motor accuracy was applied as follows. Since the index representing the motor accuracy was not defined in the past literature, the index used herein was absolute error at 50\% MVC (kgf), obtained by subtraction of the measurement pinch force value (=subject believes 50\% MVC) from target pinch force value (=prescribed $50 \% \mathrm{MVC}$ ). In addition, this index was converted to an absolute value. We measured the pinch force value using electromyogram recording software VitalRecorder2 (KISSEI COMTEC). We calculated two indexes reflecting the motor accuracy using a versatile biological analysis system, the BIMUTAS-Video (KISSEI COMTEC) (Figure 3).

The absolute error at 50\% MVC, persistence, and F/M amplitude ratio were significantly increased in the $10 \mathrm{~s}$ and 2 min group than $30 \mathrm{~s}$ and 1 min group. Add, the case of decreasing the absolute error at 50\% MVC in after than before motor imagery converged within $0.5-1.1 \%$ on $\mathrm{F} / \mathrm{M}$ amplitude ratio and $1.0-1.2 \%$ on persistence. The motor imagery effect was changed by individual motor imagery ability [14]. The important point to bring out the motor imagery effect was the quality of being distinct of motor imagery on every single person. Therefore, the motor imagery effect was dependent on the influence of previous motor practice. In this study, motor practice of $30 \mathrm{~s}$ or $1 \mathrm{~min}$ might be acquired accurate motor memory, and these subjects were doing quality of being distinct of motor imagery using accurate motor memory. Oishi et al. [15] reported that no significant differences were observed in the $\mathrm{H}$-reflex amplitude between rest and during motor imagery, only the subjects of doing quality of being distinct of motor imagery on speed skate player. Nomura et al. [16] reported that no significant differences were observed in the $\mathrm{F} / \mathrm{M}$ amplitude ratio between rest and during motor imagery, only the subjects of doing quality of being distinct of motor imagery. But, F/M amplitude ratio was significantly increased in during motor imagery than rest on the subjects of quality of being indistinct. In conclusion, these subjects of the decreasing absolute error
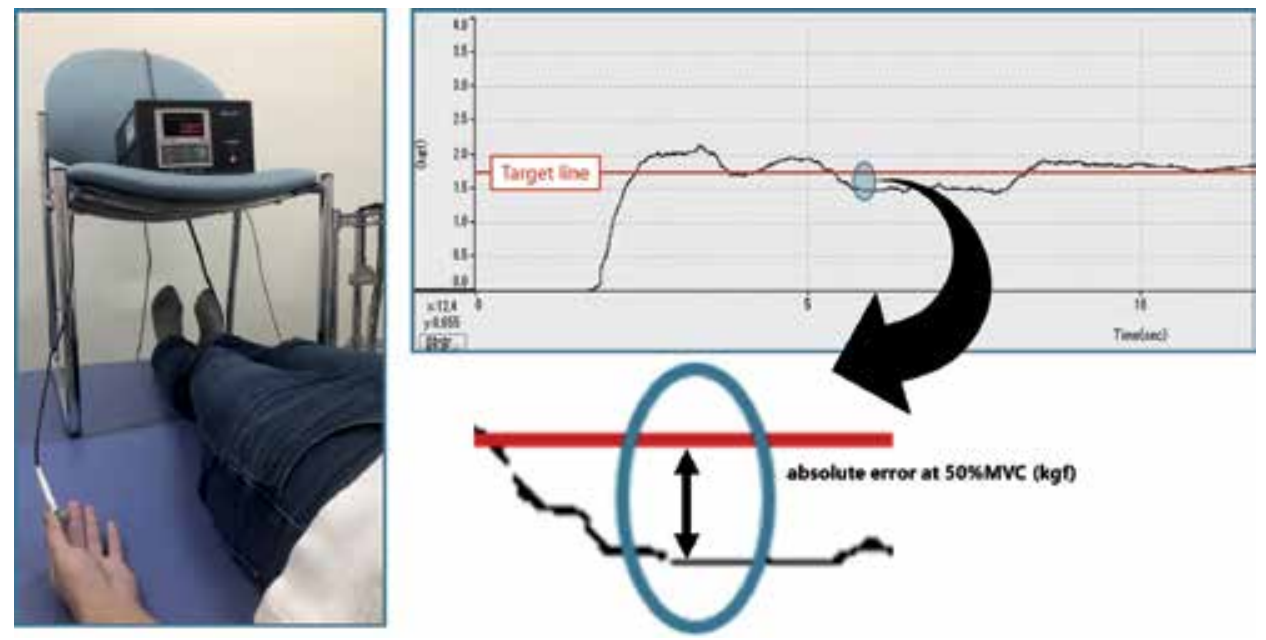

Figure 3.

Assessment method for motor accuracy. 
Effective Motor Imagery Application: Examining Spinal Cord Excitability from the F-Wave... DOI: http://dx.doi.org/10.5772/intechopen.91232

\begin{tabular}{|c|c|c|c|c|}
\hline & 10sec group & 30 sec group & Imin group & $2 \mathrm{~min}$ group \\
\hline Increase rote for $F / M$ amplitude rotio (N) & $1.6 \pm 2.4 * * t$ & $0.4 \pm 0.8$ & $0.4 \pm 0.4$ & $1.2 \pm 0.8 * \cdots$ \\
\hline Increase rate for Persistence ( 9 ) & $23 \pm 14 * \cdots$ & $87 \pm 8.4$ & $10=11$ & $20 \pm 13^{* * * *}$ \\
\hline $\begin{array}{l}\text { Increase rote for } \\
\text { Absonte error of SOSMMV (kgt) }\end{array}$ & $0.4 \pm 0.5 * 2 *$ & $0.0=0.2$ & $-0.1=0.2$ & $0.3 \pm 0.3 * \ldots$ \\
\hline
\end{tabular}

Table 2.

The result of differ motor practice time.

at 50\% MVC (=improvement motor accuracy) were doing the quality of being distinct of motor imagery based on the $30 \mathrm{~s}$ or $1 \mathrm{~min}$ motor practice. Above subjects were not necessarily overmuch increasing excitability of spinal motor neuron (Table 2).

\section{Appropriate motor practice method before motor imagery?}

\subsection{Motor imagery effect from motor practice method on the accuracy, spinal cord excitability, and autonomic nervous activity}

Our previous study [13] reported that the motor imagery after motor learning with consecutive using visual feedback was to maintain the motor accuracy. And, Heuer and Hegele [17] reported that the motor learning with intermittent using visual feedback was effective than consecutive. This section was investigated for the motor imagery effect after intermittent motor learning on motor accuracy, spinal motor neuron, and low frequency/high frequency ratio $(\mathrm{LF} / \mathrm{HF})$.

The participants were 13 healthy subjects. First, the F-wave and LF/HF were recorded at rest. Second, these subjects practiced for adjustment pinch force at $50 \%$ MVC for $30 \mathrm{~s}$ with intermittent visual feedback. Third, these subjects challenged adjustment pinch force at 50\% MVC without visual feedback, and the absolute error at 50\% MVC was assessment in this timing. Fourth, the F-wave and LF/HF were recorded during motor imagery. Finally, these subjects did pinch task again.

ANS activity was recorded using a heart rhythm scanner [Biocom Technologies; Heart Rhythm Scanner PE (Ark Trading Pacific Inc.)] (Figure 2). The pulse wave from the photoplethysmography sensor attached to the earlobe was recorded. The low frequency was reflected in sympathetic and parasympathetic nerves. The low frequency at band of $0.05-0.15 \mathrm{~Hz}$ was used. High frequency reflected only parasympathetic nerves and used band of $0.15-0.50 \mathrm{~Hz}$. Therefore, the low frequency/ high frequency (LF/HF) ratio reflected the cardiac sympathetic nerve activity [18]. This index was obtained by analyzing the pulse wave recorded by the Heart Rhythm Scanner PE. It is considered to be an index of the sympathetic nerve activity. The European Society of Cardiology and the North American Society of Pacing and Electrophysiology recommend $5 \mathrm{~min}$ recordings for heart rate variability analysis. Also, we were careful about the hours and subject condition, because the LF/HF ratio exponentially increased immediately by rising in the morning and after smoking, eating, and vigorous exercise [19].

The absolute error at 50\% MVC does not differ before and after motor imagery. However, nine subjects were decreasing for the absolute error at 50\% MVC after motor imagery. The persistence, F/M amplitude ratio, and LF/HF significantly increased during motor imagery than those at rest. This study's motor task was adjustment force in isometric contraction. But, this ability was difficult to maintain [20]. Therefore, motor accuracy decreased for the time course. But, 
in this study's result, motor accuracy was not decreasing. Due to this reason, we thought that motor imagery was maintaining motor accuracy. The differences point on this study and our previous study [13] was majority subject improvement motor accuracy. It is considered that intermittent motor learning was to increase the motor imagery effect than consecutive. Next, we thought that the F-wave's result referred to appropriate motor imagery time. The dorsolateral prefrontal cortex (DLPFC) has a role in motor cognition and has connections with the supplementary motor area and insula cortex. The anterior cingulate and insula cortices have roles in cardiovascular regulation. Transcranial magnetic stimulation (TMS) to the primary motor cortex increases skin sympathetic nerve activity [21], and transcranial direct stimulation (tDCS) to the primary motor cortex increases the $\mathrm{LF} / \mathrm{HF}$ ratio [22]. $\mathrm{tDCS}$ is a noninvasive neuromodulatory technique that has been used to influence corticospinal excitability. The activation of the SMA, pM, DLPFC, and insular cortex during motor imagery might influence primary motor cortex activity, and it is thought that the primary motor cortex activity during motor imagery stimulates the cardiac sympathetic nerve fibers via the corticospinal tract. In addition, Bunno et al. [23] reported that motor imagery was to increase the persistence, $\mathrm{F} / \mathrm{M}$ amplitude ratio, and $\mathrm{LF} / \mathrm{HF}$ ratio. This report was corresponding to the present data. And, the rostral ventromedial medulla is part of the reticulospinal tract [24] and is involved in regulation of sympathetic nerve activity and motor execution [25]. It is considered that activation of the

\begin{tabular}{lcc}
\hline \hline & rest & motor imagery \\
\hline F/M amplitude ratio (\%) & $1.4 \pm 0.7$ & $1.9 \pm 1.0^{*}$ \\
Persistence $(\%)$ & $62.2 \pm 11.7$ & $77.4 \pm 19.0^{*}$ \\
LF/HF $(\%)$ & $1.5 \pm 0.4$ & $2.9 \pm 1.5^{*}$ \\
\hline \hline Absorute error at 50\%MVC $(\mathrm{kgf})$ & Before motor imagery & After motor imagery \\
\hline \hline & $0.3 \pm 0.2$ & $0.2 \pm 0.1$ \\
\hline \hline
\end{tabular}

Table 3.

The result of motor practice effect on the method.

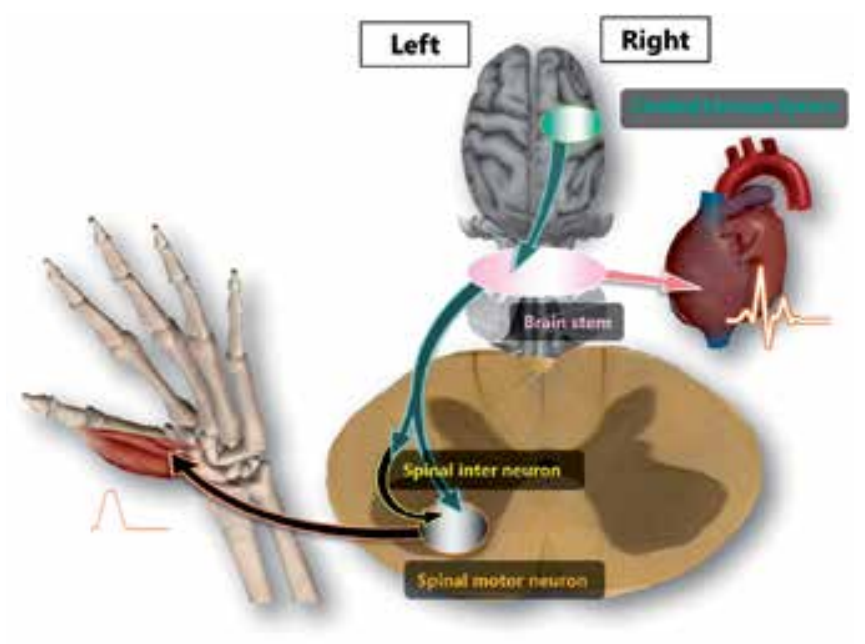

Figure 4.

Schematic model for the reason of excitability of spinal motor neuron. 
cerebral cortex during motor imagery increases cardiac sympathetic nerve activity via the corticospinal and reticulospinal tracts (Table 3, Figure 4).

\section{Appropriate doing motor imagery time?}

\subsection{Relationship between duration in the motor imagery and spinal cord excitability}

Our previous study [26] investigated continuation days for motor imagery. As a result, the motor imagery improved the motor accuracy by 3 days continuation. Our previous study [26] adopted motor imagery time for $1 \mathrm{~min}$. But, another report [23] adopted motor imagery time for $5 \mathrm{~min}$. Effective motor imagery time around once should be clear in order to apply clinical applications. We anticipate that 5 min motor imagery was a difficult continuation in doing motor imagery. In the case of above pattern, these subjects might be divided and doing motor imagery might be repeated. About this, Umeno et al. [27] reported that the repeat doing was load or burden motor imagery effect, and performance was improvement. We thought that 5 min motor imagery was not realistic. And, we expected that the motor imagery was repeated within $5 \mathrm{~min}$. Is it useful so as to be repeated? We clarified this point in this section.

The subjects were 13 healthy subjects, except those rated as lack of concentration. After doing exercise to adjust for 50\% MVC of the pinch force. Next motor imagery was taken for $5 \mathrm{~min}$, and F-waves were recorded at the first and last $1 \mathrm{~min}$. We ordered continuation doing motor imagery whenever possible within $5 \mathrm{~min}$. In the case of difficulty in this task, we additional ordered repeats doing motor imagery (Table 4).

In the result, the persistence and the amplitude $\mathrm{F} / \mathrm{M}$ ratio were not different in two periods. Activation of the primary motor area, supplementary motor area, premotor area, primary somatosensory area, dorsolateral prefrontal area, cingulate cortex, and cerebellar regions occurred during motor imagery [28-30]. Furthermore, Suzuki et al. [31] reported the excitability of spinal motor neurons in the motor imagery condition to be influenced by the descending pathways from the cerebral nervous system. We attribute this to the influence of the descending pathways corresponding to the thenar muscle. By contrast, excitatory inputs travel through the corticospinal pathway and reticulospinal tract and from the corticospinal pathway and extrapyramidal tract to anterior horn cells. Also, the spinal interneuron influences the excitability of spinal motor neuron in the motor accuracy on the hand finger [32-35]. Spinal interneuron acted spinal anterior horn cell on facilitate or inhibition $[36,37]$. In conclusion, the excitability of spinal motor neuron might be adjusted from descending pathways and spinal interneuron. The excitability of spinal motor neuron had the same influence on both the first and last $1 \mathrm{~min}$.

\begin{tabular}{lcc}
\hline \hline & First $1 \mathrm{~min}$ & Last $1 \mathrm{~min}$ \\
\hline F/M amplitude ratio (\%) & $1.8 \pm 0.4$ & $1.6 \pm 0.5$ \\
Persistence (\%) & $80.9 \pm 15.8$ & $76.5 \pm 20.6$ \\
\hline \hline
\end{tabular}

Table 4.

The result of first and last 1 min motor imagery effect. 


\section{Appropriate doing motor imagery method?}

\subsection{Motor imagery effect for the time of adjustment pinch force and spinal cord excitability based on the motor imagery method}

We use a tool and an object, manipulated by the upper limb, for activities of daily living. For example, buttoning and unbuttoning, using chopsticks, picking up coins, and so forth, are important accuracy and expeditiousness for the motor activities. We already examined the accuracy on motor imagery effect. In this study, we examined the expeditiousness on motor imagery effect. Also, we examined the effect of motor imagery method.

The participants were 15 healthy subjects. First, F-waves were recorded at rest. Second, these subjects were practiced for adjustment pinch force at 50\% MVC for $30 \mathrm{~s}$. Third, these subjects were challenged for adjustment pinch force at 50\% MVC with visual feedback, and the using time of adjustment pinch force at $50 \%$ MVC was assessment in this timing. Fourth, F-waves were recorded during motor imagery. These subjects were doing motor imagery at muscle imagery, vision imagery and composite imagery. Finally, these subjects did pinch task, and assessment for using time of adjustment pinch force at $50 \%$ MVC again.

The using time of adjustment pinch force at 50\% MVC was significantly shortening after motor imagery than before method of composite for kinesthetic and visual. The F/M amplitude ratio did not differ between rest and during motor imagery in all motor imagery methods. The case of difficult kinesthetic motor imagery was doing vision motor imagery into unconsciousness [38]. And, kinesthetic motor imagery was having high effect on the improvement motor performance than vision image [39]. Therefore, we thought that the subject of difficult doing kinesthetic motor imagery might be doing vision motor imagery, and vision motor imagery was a severe task for improving expeditiousness. But, composite for kinesthetic and visual motor imagery was improvement expeditiousness. Itou [40] reported that the subject to make efforts for doing vision imagery like numerical value during kinesthetic motor imagery was improving motor accuracy in grip task. But, the subject doing muscle motor imagery with unrelated numerical value was not improving the motor accuracy. The quality of being distinct of motor imagery was decided not only based on constant ability but also motor imagery practice [41]. The quality of being distinct of motor imagery was to converge the excitability of spinal motor neuron and improve motor performance [13]. And, our previous study [42] reported that motor imagery might improve expeditiousness. It is considered that the composite for kinesthetic and visual motor imagery might be improving quality of being distinct of motor imagery. And, the quality of being distinct of motor imagery might be shortening time of adjustment pinch force at 50\% MVC (Table 5).

\begin{tabular}{|c|c|c|}
\hline & rest & motor ïmogery \\
\hline Evoked $F / M$ amplitude ratio ( $\%$ [kinesthetic] & $0.8 \pm 0.2$ & $0.7 \pm 0.3$ \\
\hline Evoked $F / M$ amplitude rotio (\% [vision] & $0.6 \pm 0.3$ & $0.9 \pm 0.3$ \\
\hline \multirow[t]{2}{*}{ Evoked F/M omplitude rotio (\%) [composite] } & $0.8 \pm 0.2$ & $1.0 \pm 0.3$ \\
\hline & Before motor imagery & After motor imagery \\
\hline Using time of adjustment 50\%MVC (sec) [kinesthetic] & $0.9 \pm 0.3$ & $0.9 \pm 0.3$ \\
\hline Using time of adjustment 50\%MVC(sec) [vision] & $0.9 \pm 0.4$ & $0.9 \pm 0.2$ \\
\hline Using time of adjustment $509 \mathrm{MMC}(\mathrm{sec})$ [composite] & $1.0 \pm 0.5$ & $0.7 \pm 0.3^{*}$ \\
\hline
\end{tabular}

Table 5 .

The result of kinesthetic, vision, and compound imagery effect. 


\section{Motor imagery effect for the other side}

\subsection{Motor imagery effect for the opposite on the accuracy and spinal cord excitability}

The stroke patients found it difficult exactly during motor practice on the paralysis side, because these subjects had spasticity or flaccidity. This case was difficult to motor imagery effect based on our previous study result. In this study, we examined the motor imagery effect for the other hand on motor accuracy and excitability of spinal motor neuron.

A total of 20 healthy subjects were evenly allocated into two groups, one group task was adjustment pinch force at 10\% MVC; and, the other group task was 50\% MVC. F-waves were recorded at rest. Next, subjects were asked to practice for the adjustment of numerical target with right hand. Next, these subjects were asked to perform motor imagery (or without motor imagery) with left hand, and F-waves were recorded. Finally, the subjects were then asked to generate pinch force at numerical target without visual feedback, and accurate index for absolute error at $50 \%$ MVC was recorded.

No significant differences in the absolute error at $10 \%$ or $50 \%$ MVC were observed between the pinch task after motor imagery and without motor imagery. But, the absolute error in the $50 \%$ MVC was significantly more increased than in the $10 \%$ MVC. The persistence in the motor imagery of both groups was significantly more increased than in the resting condition. Only in $10 \%$ MVC group, the $\mathrm{F} / \mathrm{M}$ amplitude ratio in the motor imagery was significantly more increased than in the resting condition. Health subject's pinch force was about $6.7 \mathrm{~kg}$ [43]. The necessary pinch force was $3 \mathrm{~kg}$ on the buttoning and unbuttoning [44], and $3.8 \mathrm{~kg}$ on cap screw-engaged to an opening part of the PET bottle [45]. Therefore, we were in need of about 44-56\% MVC pinch force for smoothly doing ADL. In this study, we thought that $50 \%$ MVC pinch force was more frequently used, but 10\% MVC pinch force was not used. Jenkins [46] reported that we decided standard central point at 50-60\% MVC in task of adjustment force. We thought that $50 \%$ MVC pinch force was misrecognition to easy. But, these subjects were potentially difficult control for this pinch force. Our previous study [47] reported that motor imagery based on the false motor memory was decreasing motor performance. These subjects might be doing motor imagery based on the false motor memory in 50\% MVC task. Therefore, motor accuracy was more deteriorated in 50\% MVC than in the 10\% MVC task. Also, persistence was increased during motor imagery. But, the $\mathrm{F} / \mathrm{M}$ amplitude ratio was only increased during kinesthetic muscle motor imagery in 10\% MVC group [48]. Kinesthetic motor imagery was having high effect on the improvement of motor performance than vision image [39]. It is considered that motor imagery effect for the other hand might be not obtained in adjustment pinch force task. Also, contraction strength might be influenced for the motor imagery effect from the motor imagery method (Table 6).

\begin{tabular}{|c|c|c|c|c|c|c|}
\hline & \multicolumn{2}{|c|}{ 10006Coug } & \multicolumn{2}{|c|}{ SoNur groue } & & \\
\hline & mer & maxo inogey & $\operatorname{sen} x$ & maroinogey & & \\
\hline 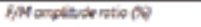 & $\sin 0.6$ & $12 n 2^{2}$ & $15 n 1.1$ & $1.1 \mathrm{n05}$ & & \\
\hline \multirow[t]{3}{*}{ 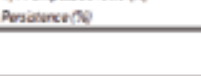 } & $\operatorname{rgn} 114$ & 4hin105: & $5+6 n 143$ & rotn12s. & & \\
\hline & \multicolumn{2}{|c|}{ 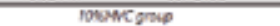 } & \multicolumn{2}{|c|}{ Sognc gnoce } & \multicolumn{2}{|c|}{ Componion belien poos } \\
\hline & nederimays & whout motar ingengy & motor inagey & wethost mothr imggey & Nown & $\sin { }^{2}$ \\
\hline 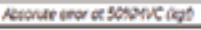 & $00 \pm 0.1$ & $0.0=00$ & $a s=0,1$ & $0.4=0.3$ & $0.0=0.1$ & $0.1 \div 02=$ \\
\hline
\end{tabular}

Table 6.

The result of motor imagery effect for the other side. 


\section{Conclusion}

Self-training may be carefulness or contraindication when it merges heart trouble and respiratory illness. Therefore, we think necessarily self-training without actual motion. Motor imagery is not limitation time, place, and using special equipment. Excitability of spinal motor neuron was increased by motor imagery. Adding effect on the breathing state, both expiration and inspiration phase on the relax respiration, was not influenced for excitability of spinal motor neuron and cardiac sympathetic nerve activity. Also, motor imagery increased excitability of spinal motor neuron. However, vividness of motor imagery was to converge a degree. Motor practice before motor imagery was important. Motor practice was appropriate for $30 \mathrm{~s}$ using intermittent visual feedback. And, for doing motor imagery, time enough was $1 \mathrm{~min}$. The motor imagery method was a recommended composite for kinesthetic and visual motor imagery. Unfortunately, motor imagery was few effects for the other hand. Therefore, motor imagery should be done on the ipsilateral side from the previous motor practice.

Combination of rehabilitation and self-training was having high effect on the improvement of a patient's performance. The self-training may be carefulness or contraindication when it merges heart trouble and respiratory illness. For their patient, motor imagery was effective, because motor imagery is not limitation time, place, and using special equipment.

\section{Conflict of interest}

Nothing.

\section{Author details}

Yuki Fukumoto

Clinical Physical Therapy Laboratory, Kansai University of Health Sciences, Osaka, Japan

*Address all correspondence to: fukumoto_3197@yahoo.co.jp

IntechOpen

(C) 2020 The Author(s). Licensee IntechOpen. This chapter is distributed under the terms of the Creative Commons Attribution License (http://creativecommons.org/licenses/ by/3.0), which permits unrestricted use, distribution, and reproduction in any medium, provided the original work is properly cited. (cc) BY 
Effective Motor Imagery Application: Examining Spinal Cord Excitability from the F-Wave...

DOI: http://dx.doi.org/10.5772/intechopen.91232

\section{References}

[1] Britton M, Andersson A. Home rehabilitation after stroke. Reviewing the scientific evidence on effects and costs. International Journal of Technology Assessment in Health Care. 2000;16:842-848. DOI: 10.1017/ s0266462300102119

[2] Farah MJ. The neural basis of mental imagery. Trends in Neurosciences. 1989;12(10):395-399. DOI: 10.1016/0166-2236(89)90079-9

[3] Liu H, Song L, Zhang T. Mental practice combined with physical practice to enhance hand recovery in stroke patients. Behavioural Neurology. 2014;2014:876416. DOI: $10.1155 / 2014 / 876416$

[4] Yue G, Cole KJ. Strength increases from of motor program: Comparison of training with maximal voluntary and imagined muscle contractions. Journal of Neurophysiology. 1992;67:1114-1123. DOI: 10.1152/jn.1992.67.5.1114

[5] Beyer L, Weiss T, Hansen E, et al. Dynamics of central nervous activation during motor imagination. International Journal of Psychophysiology. 1990;9:75-80. DOI: 10.1016/0167-8760(90)90008-2

[6] Decety J, Jeannerod M, Germain M, et al. Vegetative response during imagined movement is proportional to mental effort. Behavioural Brain Research. 1991;42:1-5. DOI: 10.1016/ s0166-4328(05)80033-6

[7] Bolliet O, Collet C, Dittmar A. Autonomic nervous system activity during actual and mentally simulated preparation for movement. Applied Psychophysiology and Biofeedback. 2005;30:11-20. DOI: $10.1007 /$ s10484-005-2170-2

[8] Ozaki I, Kurata K. The effects of voluntary control of respiration on the excitability of the primary motor hand area, evaluated by end-tidal $\mathrm{CO}_{2}$ monitoring. Clinical Neurophysiology. 2015;126:2162-2169. DOI: 10.1016/j. clinph.2014.12.032

[9] Sparing R, Dafotakis M, Buelte D, et al. Excitability of human motor and visual cortex before, during, and after hyperventilation. Journal of Applied Physiology. 2007;102:406-411. DOI: 10.1152/japplphysiol.00770.2006

[10] Iwamoto E, Iwata M, Sekikawa K, et al. Influence of control of inspiratoryexpiratory ratio on coordination between locomotor and respiratory rhythms during walking. Japan Society of Physiological Anthropology. 2010;15(1):1-8. DOI: $10.20718 /$ jjpa.15.1_1 [in Japanese]

[11] Guz A. Brain breathing and breathlessness. Respiration Physiology. 1997;109:197-204. DOI: 10.1016/ S0034-5687(97)00050-9

[12] Gandevia SC, Rothwell JC. Activation of the human diaphragm from the motor cortex. The Journal of Physiology. 1987;384:109-118. DOI: 10.1113/jphysiol.1987.sp016445

[13] Fukumoto Y, Bunno Y. The effects of motor imagery after a variety of motor learning times on excitability of spinal motor neurons and accurate. In: Suzuki T, editor. Neurological Physical Therapy. Croatia: Intech; 2017. pp. 71-94. DOI: $10.5772 / 67470$

[14] Nishida T, Katsube A, Inomata K, et al. A factor analytical study on vividness of motor imagery. Taiikugaku Kenkyu (Japan Journal of Physical Education, Health and Sport Sciences). 1981;26(3):189-205. DOI: 0.5432/ jjpehss.KJ00003402682 [in Japanese]

[15] Oishi K, Kimura M, Yasukawa M, et al. Changes of 
physiological parameters during mental rehearsal of speed skating. Japanese Society of Physical Education. 1992;36:303-312. DOI: 10.5432/jjpehss. KJ00003391834 [in Japanese]

[16] Nomura M, Maeda T, Kado N, et al. Effects of individual differences in motor imagery ability on the excitability of spinal neural function. Rigakuryoho Kagaku. 2016;32(2):195-199. DOI: 10.1589/rika.32.195 [in Japanese]

[17] Heuer H, Hegele M. Constraints on visuo-motor adaptation depend on the type of visual feedback during practice. Experimental Brain Research. 2008;185:101-110. DOI: 10.1007/ s00221-007-1135-5

[18] Malik M, Bigger JT, Camm AJ, et al. Heart rate variability: Standards of measurement, physiological interpretation and clinical use. European Heart Journal. 1996;17(3):354-381. DOI: $10.1093 /$ oxfordjournals.eurheartj.a014868

[19] Tochikubo O, Kawano Y, Miyajima E, et al. Circadian variation of hemodynamics and baroreflex functions in patients with essential hypertension. Hypertension Research. 1997;20(3):157166. DOI: 10.1291/hypres.20.157

[20] Ohashi Y. A study for retention characteristics of isometric force information. Japanese Physical Therapy Association. 1993;20(6):355-359. DOI: 10.15063/rigaku.KJ00003128108 [in Japanese]

[21] Silber DH, Sinoway LI, Leuenberger UA, et al. Magnetic stimulation of the human motor cortex evokes skin sympathetic nerve activity. Journal of Applied Physiology. 2000;88:126-134. DOI: 10.1152/ jappl.2000.88.1.126

[22] Clancy JA, Johnson R, Raw R, et al. Anodal transcranial direct current stimulation (tDCS) over the motor cortex increases sympathetic nerve activity. Brain Stimulation. 2014;7: 97-104. DOI: 10.1016/j.brs.2013.08.005

[23] Bunno Y, Suzuki T, Iwatsuki H. Motor imagery muscle contraction strength influences spinal motor neuron excitability and cardiac sympathetic nerve activity. Journal of Physical Therapy Science. 2015;27:3793-3798. DOI: $10.1589 /$ jpts.27.3793

[24] Kerman IA, Enquist LW, Watson SJ, et al. Brainstem substrates of sympatho motor circuitry identified using trans-synaptic tracing with pseudorabies virus recombinants. The Journal of Neuroscience. 2003;23:4657-4666. DOI: 10.1523/ JNEUROSCI.23-11-04657.2003

[25] Allen GV, Cechetto DF.

Serotoninergic and nonserotoninergic neurons in the medullary raphe system have axon collateral projections to autonomic and somatic cell groups in the medulla and spinal cord. The Journal of Comparative Neurology. 1994;350:357-366. DOI: 10.1002/ cne. 903500303

[26] Imanara T, Fukumoto Y, Suzuki T. The effect of continuous motor imagery on the accuracy of movement and the excitability of spinal neural function: A 5-day study of a single participant. Journal of Kansai Physical Therapy. 2017;17:77-84. DOI: 10.11354/jkpt.17.77 [in Japanese]

[27] Umeno K, Nakamura K, Inomoto A, et al. Relationships between motor imagery ability evaluated using various methods and the effect of mental practice. Rigakuryoho Kagaku. 2017;33(2):313-317. DOI: 10.1589/ rika.33.313 [in Japanese]

[28] Stephan KM, Fink GR, Passingham RE, et al. Functional anatomy of the mental representation of upper extremity movements in healthy subjects. Journal of Neurophysiology. 
1995;73:373-386. DOI: $10.1152 /$ jn.1995.73.1.373

[29] Roland PE, Larsen B, Lassen NA, et al. Supplementary motor area and other cortical areas in organization of voluntary movements in man. Journal of Neurophysiology. 1980;43:118-136. DOI: 10.1152/jn.1980.43.1.118

[30] Lotze M, Montoya P, Erb M, et al. Activation of cortical and cerebellar motor areas during executed and imagined hand movements: An fMRI study. Journal of Cognitive Neuroscience. 1999;11:491-501. DOI: 10.1162/089892999563553

[31] Suzuki T, Bunno Y, Onigata C, et al. Excitability of spinal neural function by motor imagery with isometric opponens pollicis activity: Influence of vision during motor imagery. NeuroRehabilitation. 2014;34:725-729. DOI: $10.3233 / N R E-141085$

[32] Kinoshita M, Matsui R, Kato S, et al. Genetic dissection of the circuit for hand dexterity in primates. Nature. 2012;487:235-238. DOI: 10.1038/ nature11206

[33] Takei T, Seki K. Spinal interneurons facilitate coactivation of hand muscles during a precision grip task in monkeys. Journal of Neuroscience. 2010;30:17041-17050. DOI: 10.1523/ JNEUROSCI.4297-10.2010

[34] Takei T, Seki K. Spinal premotor interneurons mediate dynamic and static motor commands for precision grip in monkeys. Journal of Neuroscience. 2013;33:8850-8860. DOI: 10.1523/JNEUROSCI.4032-12.2013

[35] Alstermark B, Isa T, Ohki Y, et al. Disynaptic pyramidal excitation in forelimb motoneurons mediated via C3-C4 propriospinal neurons in the Macaca fuscata. Journal of Neurophysiology. 1999;82:3580-3585. DOI: 10.1152/jn.1999.82.6.3580
[36] Pauvert V, Pierrot-Deseilligny E, Rothwell JC, et al. Role of spinal premotoneurones in mediating corticospinal input to forearm motoneurons in man. Journal of Physiology (London). 1998;508:301-312. DOI: 10.1111/j.1469-7793.1998.301br.x

[37] Pierrot-Deseilligny E. Propriospinal transmission of part of the corticospinal excitation in humans. Muscle \& Nerve. 2002;26:155-172. DOI: 10.1002/ mus.1240

[38] Diewert GL. The role of vision and kinaesthesis in coding of two dimensional movement information. Journal of Human Movement Studies. 1976;3:191-198. DOI: 10.1007/978-0-387-77064-2_16

[39] Ridderinkhof KR, Brass M. How Kinesthetic motor imagery works: A predictive-processing theory of visualization in sports and motor expertise. Journal of Physiology, Paris. 2015;109:53-63. DOI: 10.1016/j. jphysparis.2015.02.003

[40] Ito M. The effect of covert rehearsal and the response biasings by mental activities in short-terrn retention of force information. Japanese Society of Physical Education. 1984;29(3):207-216. DOI: 10.5432/jjpehss.KJ00003392147 [in Japanese]

[41] Ito M. A field study on the effects of active and passive image rehearsals on observational learning of breast stroke skills. Japanese Society of Physical Education. 1980;24(4):291-299. DOI: 10.5432/jjpehss.KJ00003402488 [in Japanese]

[42] Fukumoto Y, Suzuki T, Iwatsuki H. Effects of motor imagery on accuracy, expeditiously and excitability of spinal anterior horn cell. Japanese Journal of Clinical Neurophysiology. 2019;47(1):23-33. DOI: 10.4172/21657025.1000339 [in Japanese] 
[43] Takaoka A, Maki T, Masuyama S, et al. Pinch force on health subject.

Japanese Occupational Therapy

Research. 1985;4(1):47-52 [translated

from Japanese]

[44] Hisano T, Tsuneoka T, Imamura $\mathrm{H}$, et al. Opponensplasty by the

Camitz's method. Orthopedics and Traumatology. 1986;34(4):1474-1478. DOI: $10.5035 /$ nishiseisai.34.1474 [in Japanese]

[45] Suzuki M, Hirano D, Ogano M, et al. Analysis of behavior in opening plastic bottle caps among healthy women: Differences between young and elderly individuals. Journal of the International University of Health and Welfare. 2017;22(2):37-45 [in Japanese]

[46] Jenkins WO. The discrimination and reproduction of motor adjustments with various types of aircraft controls. American Journal of Psychology. 1947;60:397-406

[47] Fukumoto Y, Take N, Fuchimoto M, et al. Effect of motor imagery on the excitability of the spinal nerve function and its impact on the accuracy of movement. Journal of Kansai Physical Therapy. 2015;15:79-84. DOI: 10.11354/ jkpt.15.79 [in Japanese]

[48] Bunno Y. Imagery strategy affects spinal motor neuron excitability: Using kinesthetic and somatosensory imagery. Neuroreport. 2019;30:463-467. DOI: 10.1097/WNR.0000000000001218 


\title{
Excitability of Spinal Motor Neurons in the Upper Extremity during Voluntary Movement with Different Difficult Tasks in the Lower Extremity
}

\author{
Naoki Kado
}

\begin{abstract}
The purpose of this study was to examine the relationship between excitability of the spinal motor neurons in the upper extremity and difficulty of tasks applied to the lower extremities. Twenty healthy volunteers agreed to participate. Our findings suggest that the excitability of spinal motor neurons in the right arm was increased during voluntary movement of the lower extremities. Excitability of the spinal motor neurons might be increased by the facilitatory effect at the spinal and cortical levels. Regarding the facilitatory effects at the cortical level, the effects of difficult tasks might be larger than those of simple tasks, but the most difficult task might be affected by the facilitatory and inhibitory effects of the central nervous system.
\end{abstract}

Keywords: F-wave, spinal motor neuron, remote muscle, leg movement, task difficulty

\section{Introduction}

It is important to understand the effects of remote muscle contractions on other muscles during physical therapy. For example, associative reactions observed in hemiplegic patients with cerebrovascular disorders include tonic reflexes from the muscle groups of one limb to those of the other limb. Associative reactions often occur during or before the start of exercise and worsen abnormal synergic movements, making selective exercises difficult in the upper extremities of the paralyzed side. In physical therapy, it is necessary to reduce associative reactions, perform selective exercises, and evaluate the exercise types that trigger such associative reactions. Furthermore, the facilitatory and inhibitory effects of muscle contractions in remote regions can be used to manipulate central nervous system input and output during physical therapy. As such, to enable the efficacy of physical therapy, the neurophysiological effects of muscle contractions of remote regions on other muscles must be examined.

The mechanism of the facilitatory effect of muscle contractions of remote regions has been analyzed using $\mathrm{H}$-waves, F-waves, and motor-evoked potentials induced by transcranial magnetic stimulation [1-6]. The Jendrassik maneuver, a 
method for enhancing the reflexes, is widely known in clinical practice. The facilitatory effects of this maneuver on the cerebral cortex and spinal motor neurons differ between the upper and lower extremities [3] and are affected by the timing from the start of exercise [2]. Furthermore, studies have shown that the facilitatory effects of the contraction of muscles in remote regions, such as the upper and lower extremities, on other regions are affected by contraction intensity $[4,5]$ and the number of muscle spindles $[5,6]$.

Some reported cases of hemiplegic patients with cerebrovascular disorders undergoing physical therapy did not differ significantly in terms of contraction strength of the muscles involved in the movements or limb position. However, the appearance of associative reactions in the upper extremities of the paralyzed side seems to depend on exercise task difficulty. In our previous study, we reported that more difficult voluntary movements of the upper extremities increased the excitability of spinal motor neurons in the contralateral upper extremities [7]. However, because there are cases in which associative reactions appear when voluntary movements of the lower extremities such as stepping are induced, the excitability of spinal motor neurons in the upper extremity during movement of the lower extremities requires examination. In this study, we investigated the effect of differences in the difficulty of lower extremity movement tasks on the excitability of upper extremity spinal motor neurons using F-waves evoked by electromyography (EMG).

\section{Subjects and methods}

\subsection{Participants}

Twenty healthy right-footed adults (17 men, 3 women; mean age, $25.7 \pm 6.2$ years) with no orthopedic or neurological abnormalities participated in this study. To determine each participant's dominant foot, we used the dominant foot test described by Chapman et al. [8]. From the test results, those with a total score $\geq 28$ on the 11 items were identified as left-footed and excluded from the study.

In addition to receiving explanations of the study objectives, the subjects were informed that their test data would be strictly confidential and that they could withdraw from the study at any time. Subjects' signatures on the study consent forms were obtained once they had agreed to participate. This study received approval from the ethics committee of Kobe College of Rehabilitation.

\subsection{Conditions for recording the F-wave}

F-waves were derived from the right abductor pollicis brevis (APB) muscle during the rest and motor tasks of the bilateral lower limbs using the Viking Quest EMG system (Nicolet Biomedical, Madison, WI, USA; Figure 1). Each subject was seated on a chair during the test with the neck flexed at $30^{\circ}$; hip flexed at $80^{\circ}$; knee joints flexed at $90^{\circ}$; and the ankle, shoulder, elbow, and hand joints at $0^{\circ}$.

Stimulation conditions for F-wave elicitation were 30 consecutive stimulations of the median nerve at the right wrist with an intensity of $120 \%$ of the stimulation intensity required to evoke the maximum $\mathrm{M}$-wave, a duration of $0.2 \mathrm{~ms}$, and a frequency of $0.5 \mathrm{~Hz}$. Bipolar surface-stimulating electrodes used in general motor nerve conduction velocity tests were used as the stimulating electrodes with the cathode at the center and the anode at the periphery. For the recording conditions, 
Excitability of Spinal Motor Neurons in the Upper Extremity during Voluntary Movement... DOI: http://dx.doi.org/10.5772/intechopen.91724

the exploring electrode was placed over the belly of the right APB muscle, the reference electrode on the proximal phalanx of the thumb, and the ground electrode on the forearm (Figure 2).

The electrode sites were rubbed with a cotton pad that was moistened with alcohol to remove any oil and the horny layer of the skin was removed using a skin preparation gel. Ten-millimeter-diameter silver-silver chloride disk electrodes were affixed to the skin surfaces using a conductive paste. Bioelectrical signals obtained at the electrodes were conducted to the input via lead wires, amplified with a bioamplifier, converted from analog to digital signals with an AD converter, and displayed on a personal computer as waveforms. All signals were digitized at a sampling frequency of $24 \mathrm{kHz}$ and recorded on the hard disk. The data were bandpass filtered between 20 and $3 \mathrm{kHz}$ with a sweep speed of $5 \mathrm{~ms} / \mathrm{div}$ and amplitude sensitivity of $200 \mu \mathrm{V} /$ div.

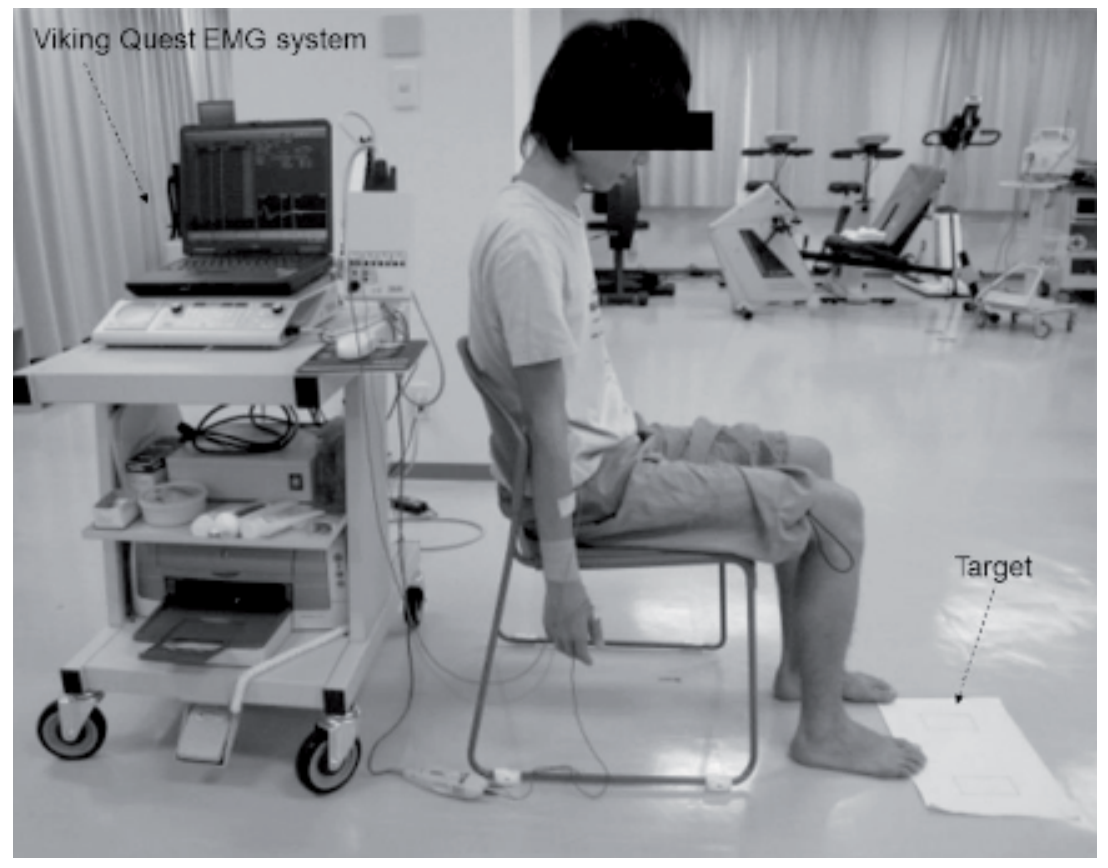

Figure 1.

Measurement of the F-wave.

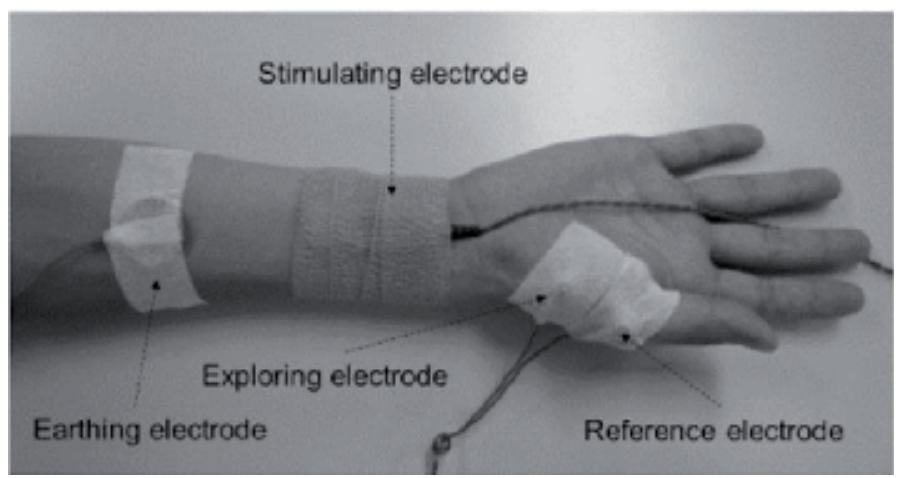

Figure 2.

Placement of the electrode. 
The F-waves were analyzed for the amplitude ratio of the F/M. The amplitude ratio of the F/M was calculated as the ratio of the average peak-to-peak F-wave amplitude and the maximum $\mathrm{M}$-wave amplitude.

\subsection{Motor tasks}

There are cases of stepping exercise to a specified space in physical therapy. This ability is necessary when avoiding obstacles or walking in defined places. In this study, the following different difficult tasks were performed.

In the lower extremity movement task, the participants stepped alternately to the left and right under four different difficulty levels. The difficulty index was defined by the exercise distance and target width, and the exercise duration was proportional to it [9]. Because exercise speed and distance can affect the excitability of the spinal motor neurons in the upper extremity, we manipulated the degrees of task difficulty by changing the target width (Figure 3). In Task 1 , the participants' feet landed randomly. In Task 2, two $7.5 \mathrm{~cm} \times 10.0 \mathrm{~cm}$ (width $\times$ length) targets were set and the participants were asked to land on the targets with the thumb of the foot. In Task 3, two $5.0 \mathrm{~cm} \times 10.0 \mathrm{~cm}$ targets were set and the participants were asked to land on the targets with their big toe. In Task 4, two $2.5 \mathrm{~cm} \times 10.0 \mathrm{~cm}$ targets were set and the participants were asked to land on the targets with their big toe. These target widths were set two and three times based on the width of big toe, respectively (i.e., $2.5,5.0$, and $7.5 \mathrm{~cm}$ ). There were $20.0-\mathrm{cm}$ intervals between targets. The exercise frequency was $1 \mathrm{~Hz}$. Each task was performed on the right and left lower extremities. The order of the tasks was randomized with a 1-minute rest between tasks. During the lower extremity movement task, electrical stimulation for F-wave derivation was given when the hip joint was adducted.

The success rate of each task and motor activity of the lower extremities were investigated using surface EMG in five adult men (mean age, $24.6 \pm 3.5$ years) before the exercise tasks. Surface EMG was performed using the MQ8 telemetry

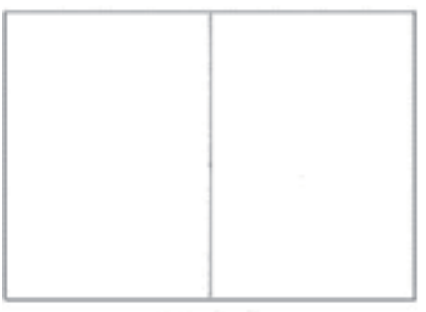

Task 1

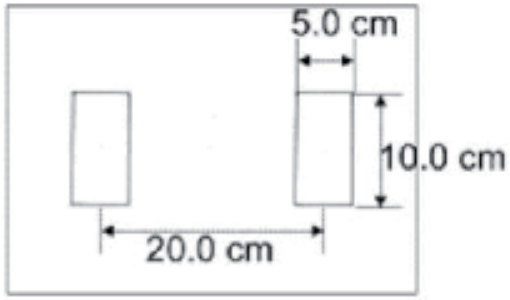

Task 3

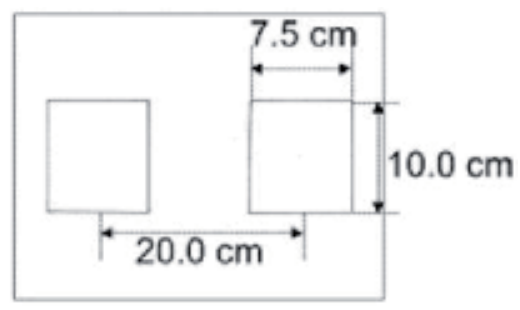

Task 2

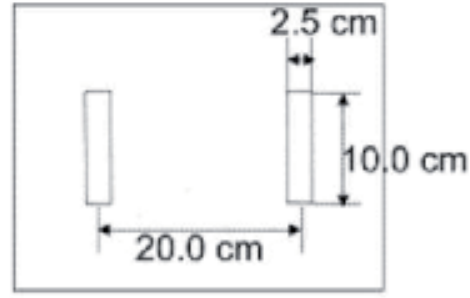

Task 4

Figure 3 .

Task targets. 
Excitability of Spinal Motor Neurons in the Upper Extremity during Voluntary Movement... DOI: http://dx.doi.org/10.5772/intechopen.91724

\begin{tabular}{cccc}
\hline \multirow{2}{*}{ Motor task } & \multicolumn{2}{c}{ Relative value of iEMG } & \multirow{2}{*}{ Suceess rate $(\%)$} \\
\cline { 2 - 3 } Rectus fenoris & Adductor of hip joint & \\
Task 1 & $1.16(1.06-1.46)$ & $1.02(1.01-1.02)$ & \\
Task 2 & $1.35(1.11-1.46)$ & $1.02(1.01-1.03)$ & 100.0 \\
Task 3 & $1.45(1.11-2.54)$ & $1.09(1.02-1.12)$ & 86.4 \\
Task 4 & $1.44(1.15-2.57)$ & $1.03(1.01-1.04)$ & 40.2 \\
Left leg & & & \\
Task 1 & $1.24(1.01-2.35)$ & $0.98(0.97-1.06)$ & \\
Task 2 & $1.32(0.92-2.58)$ & $1.01(0.98-1.45)$ & 99.2 \\
Task 3 & $1.39(1.23-3.12)$ & $1.04(0.98-1.46)$ & 81.0 \\
Task 4 & $1.50(0.81-3.00)$ & $1.04(0.96-1.49)$ & 30.2 \\
\hline Relative integrated electronnyography (iEMG) values are shown as median (interquartile range).
\end{tabular}

Table 1.

Relative value of $i E M G$ and success rates.

EMG measuring system (KISSEICOMTEC; Matsumoto, Nagano, Japan). The recording muscles were the rectus femoris and adductor muscles of the hip joint. The derivation method was bipolar, and the electrodes were positioned $2 \mathrm{~cm}$ apart. The electrode on the adductor of the hip joint was placed at the midpoint of the line that connected the pubic symphysis and the medial epicondyle of the femur, while the electrode on the rectus femoris was placed $15 \mathrm{~cm}$ above the patella. The earthing electrode was placed on the patella. The skin where the electrodes were placed was cleaned with alcohol. The electrodes were disposable. The EMG signal analysis program BIMUTAS-Video (KISSEICOMTEC) was used to analyze the derived signal. The analysis item of the surface EMG was the relative value of the integrated EMG (iEMG). The relative value of $i E M G$ was calculated from each task based on the iEMG of the sitting position. The success rate was determined from images taken using an IXY digital camera (Canon; Ota, Tokyo, Japan) that were synchronized with surface EMG. The relative values of iEMG were compared using Friedman's test. Statistical significance was accepted for values of $p<0.05$.

Table 1 shows the success rate of each task and relative value of iEMG of the muscle groups of the lower extremities. The relative value of iEMG of the rectus femoris and adductor of hip joint did not differ significantly among the tasks for either lower extremity. These results show that the tasks used in this study differed in the degree of difficulty but not in the activities of the muscle groups that were involved in the exercises. In this way, there was no difference in muscle activities because exercise speed and distance were similar for all tasks.

\subsection{Statistical analysis}

The amplitude ratios of the $\mathrm{F} / \mathrm{M}$ during the rest and movement tasks were compared using Dunnett's test. Statistical significance was accepted for values of $\mathrm{p}<0.05$.

\section{Results}

Figure 4 shows a typical waveform of the F-wave, while Table 2 shows changes in the amplitude ratio of the F/M for each task. 


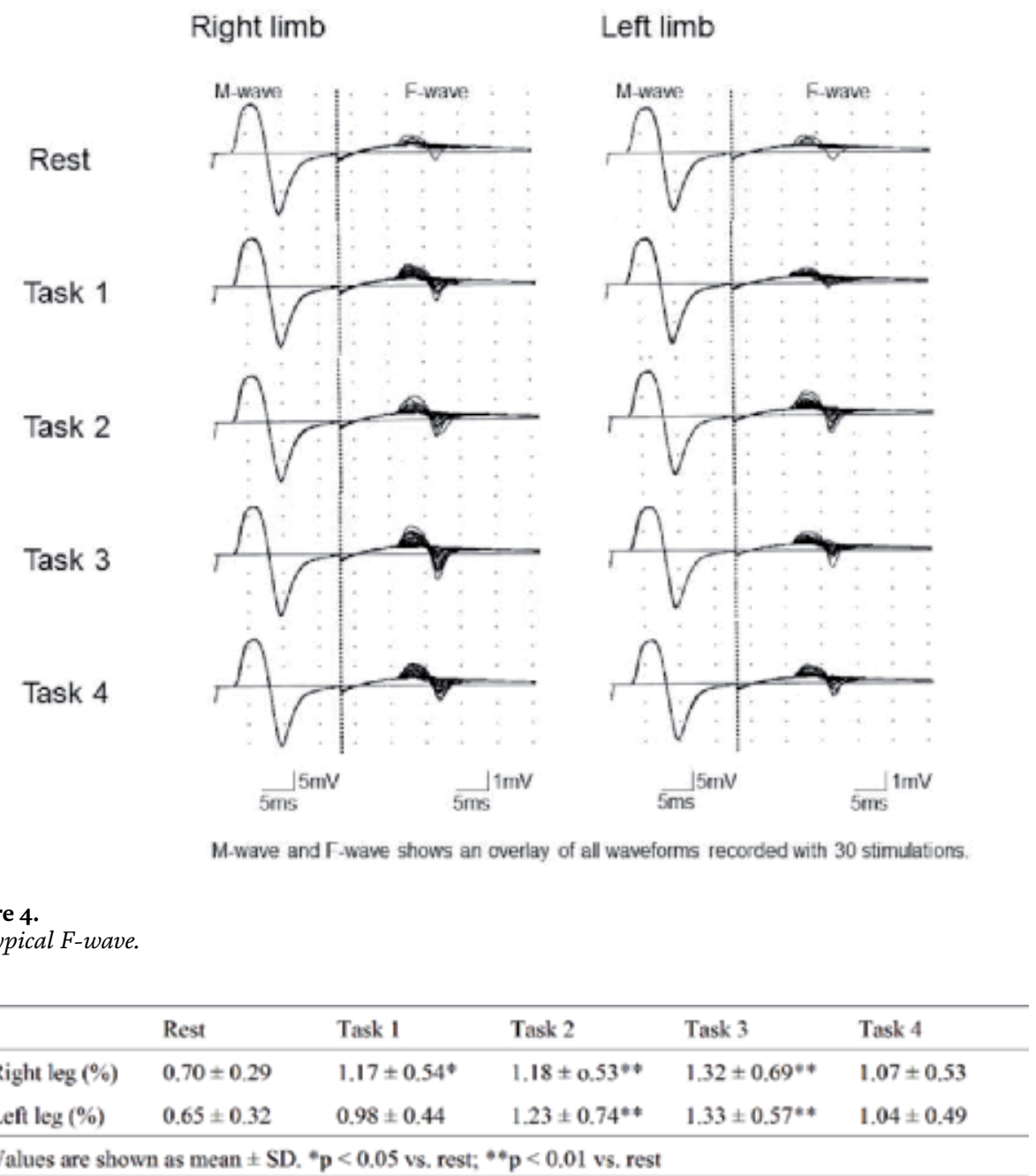

Table 2.

Change in the amplitude ratio of the F/M during lower extremity movement by task.

During the right lower extremity tasks, the amplitude ratio of the F/M increased significantly in Task 1, 2, and 3 compared to that at rest. During the left lower extremity tasks, the amplitude ratio of the F/M increased significantly in Task 2 and 3 compared to that at rest.

\section{Discussion}

F-waves originate from retrograde excitations of the $\alpha$ motor neurons through the stimulation of peripheral motor nerve axons [10]. The amplitude ratio of the F/M represents the percentage of motoneurons activated by antidromic stimulation [11]. Therefore, they were applied as a test for the excitability of spinal motor neuron pools and upper nerve systems. In this study, the amplitude ratio of the F/M of APB muscle increased in Task 1, 2, and 3 for the right lower extremities and in Task 2 and 3 for the left lower extremities. This finding suggests that the voluntary movement of the lower extremities increases the excitability of the spinal motor neurons in the upper extremity. 
Sensory input from the muscles is suggested to be involved in the facilitatory effects from the lower to upper extremities $[12,13]$. However, the excitability of the cerebral cortex and spinal motor neurons reportedly varies even in motor imagery without muscle contraction [14-16]. Hess et al. [17] studied amputees with phantom limb syndrome and reported increased contralateral motor-evoked potentials through transcranial magnetic stimulation from images of the muscle contractions of the amputated limb. This revealed the involvement of facilitatory effects by intracortical mechanisms. Furthermore, Baldissera et al. [18] reported that the excitability of the forearm region fluctuated with ankle movement by discovering that suppressing the area of the motor cortex controlling the forearm by magnetic stimulation eliminated fluctuations in the $\mathrm{H}$-waves of the radial carpal flexors accompanying the plantar/dorsiflexion motion of the ankle. Studies using functional magnetic resonance imaging and positron tomography also reported that the cerebral cortex has common activated sites during hand and foot movements $[19,20]$. Similarly, in this study, the excitability of the spinal motor neurons in the upper extremity may have increased due to proprioceptive input from voluntary movements of the lower extremities and facilitation effects from the upper central nervous system from movement recall. The effects of the proprioceptive input include excitatory effects from an increase in efferent impulses through the brainstem and cortex as well as those via the propriospinal tract. In terms of the influence of the upper central nervous system, the motor regions activated during lower extremity movement are most likely also affected during upper extremity movement via the association fibers. Because the amplitude ratio of the $\mathrm{F} / \mathrm{M}$ of the right $\mathrm{APB}$ muscle also increased during exercises involving the left lower extremities on the contralateral side, the facilitatory effect described above affected the contralateral upper extremity spinal motor neurons via the commissural fibers and non-crossed projection fibers.

Activities of the lower extremities tended to increase the spinal motor neuron excitability during the motor task. On the other hand, the results differed between the tasks. There was no significant difference in the amplitude ratio of the F/M during Task 4 for the right lower extremities or during Task 1 or 4 for the left lower extremities compared with that during rest. Because all tasks had the same exercise speed, range, and activity of the involved muscle groups, differences in exercise style corresponding to task difficulty were considered related. Voluntary movements can be simple or complex and are performed consciously or unconsciously. Kitamura et al. [21] compared simple and complex movements using movementrelated potentials and reported that supplemental and sensory-motor areas were more active in complex than in simple movements. Winstein et al. [22] reported that the activities in areas related to the planning of complex movements requiring visuomotor processing increased as the task difficulty increased using positron emission tomography. In this way, motor-related areas are more activated by difficult exercises. The cerebellum plays an important role in regulating movement. Ugawa et al. [23] reported that applying magnetic stimulation to the cerebral cortex after applying electrical stimulation to the cerebellum reduced motor-evoked potentials for a few seconds. This inhibitory effect is thought to affect the cerebral motor area via the cerebello-thalamo-cortical pathway. Furthermore, neuronal activities at other sites may be reduced because more attention is needed to improve movement accuracy [24]. This study demonstrated that it is difficult to clearly explain the excitatory and inhibitory effects on the spinal motor neurons in the upper extremity, but the following points were considered possible factors affected by differences in exercise style. The participants were able to exercise more autonomously in Task 1 than in the other tasks, which suggests involvement of the excitatory effect of proprioceptive input in the spinal cord. Task 2 and 3 required more difficult movements than Task 1, which suggests that the upper central nervous system was involved in 
addition to proprioceptive input. Task 4 of the most difficult task required greater accuracy than the other tasks, which suggests involvement of the cerebellum and cerebral cortex in addition to the abovementioned excitatory effect.

There was a difference in the results of Task 1 between the motor tasks of the right and left lower extremities. In the right lower extremities, because the amplitude ratio of the $\mathrm{F} / \mathrm{M}$ of the right $\mathrm{APB}$ muscle increased in Task 1 compared to that at rest, the excitatory effect on the spinal motor neurons in the upper extremity was considered superior to that of the contralateral lower extremities during voluntary movements of the lower leg.

\section{Conclusions}

In this study, we used F-waves to investigate the effect of different degrees of motor task difficulty of the lower extremities on the excitability of the spinal motor neurons in the upper extremity. We found that both lower extremities tended to increase the excitability of the spinal motor neurons in the upper extremity during motor tasks. This was due to the effect of proprioception on voluntary movements of the lower extremities and increased excitability of the spinal motor neurons in the upper extremity from the excitation of the upper central nervous system. Regarding the differences in task difficulty, the effect on the spinal motor neurons in the upper extremity was greater in tasks that required accurate voluntary movements, such as Task 2 and 3, than in those that could be performed autonomously, such as Task 1. However, for Task 4, which required the greatest degree of accuracy, both inhibitory effects related to the adjustment of movements and excitatory effects were involved.

When inducing voluntary movement of the lower extremities in physical therapy, the potential effects of differences in task difficulty on the facilitation and inhibition of the spinal motor neurons in the upper extremity must be considered. However, the excitability of the reflex arch of the spinal cord is different in hemiplegic patients with cerebrovascular disorders than in healthy individuals. Because the excitatory effects are expected to be significant, further studies are required to validate our results.

\section{Acknowledgements}

The author thanks M. Ito for assisting with the experiments.

\section{Conflict of interest}

The author declares no conflicts of interest. 
Excitability of Spinal Motor Neurons in the Upper Extremity during Voluntary Movement... DOI: http://dx.doi.org/10.5772/intechopen.91724

\section{Author details}

Naoki Kado

Department of Physical Therapy, Kobe College of Rehabilitation and Welfare, Hyogo, Japan

*Address all correspondence to: kado@sumire-academy.ac.jp

\section{IntechOpen}

(C) 2020 The Author(s). Licensee IntechOpen. This chapter is distributed under the terms of the Creative Commons Attribution License (http://creativecommons.org/licenses/ by/3.0), which permits unrestricted use, distribution, and reproduction in any medium, provided the original work is properly cited. (cc) BY 


\section{References}

[1] Bussel B, Morin C, PierrotDeseilligny E. Mechanism of monosynaptic reflex reinforcement during Jendrassik maneuver in man. Journal of Neurology, Neurosurgery, and Psychiatry. 1978;41(1):40-44. DOI: 10.1136/jnnp.41.1.40

[2] Kawamura T, Watanabe S. Timing as a prominent factor of the Jendrassik maneuver on the $\mathrm{H}$ reflex. Journal of Neurology, Neurosurgery, and Psychiatry. 1975;38(5):508-516. DOI: 10.1136/jnnp.38.5.508

[3] Boroojerdi B, Battaglia F, Muellbacher W, Cohen LG. Voluntary teeth clenching facilitates human motor system excitability. Clinical Neurophysiology. 2000;111(6):988-993. DOI: 10.1016/S1388-2457(00)00279-0

[4] Muellbacher W, Facchini S, Boroojerdi B, Hallett M. Changes in motor cortex excitability during ipsilateral hand muscle activation in humans. Clinical Neurophysiology. 2000;111(2):344-349. DOI: 10.1016/ S1388-2457(99)00243-6

[5] Inada T, Funakoshi K, Higashi T, Sakakibara A, Ohgi S, Funase K. Facilitation of the contralateral soleus motoneuron excitability induced by ankle flexion (in Japanese). The Journal of Japanese Physical Therapy Association. 2002;29:123-127. DOI: 10.15063/rigaku.KJ00001310746

[6] Hayashi A, Konopacki RA, Hunker CJ. Remote facilitation of $\mathrm{H}$-reflex during voluntary contraction of orofacial and limb muscles. In:

Stelmach GE, Requin J, editors. Tutorials in Motor Behavior II. Amsterdam: Elsevier; 1992. pp. 475-482

[7] Kado N, Ito M, Suzuki T, Ando H. Excitability of spinal motor neurons in the contralateral arm during voluntary arm movements of various difficulty levels. Journal of Physical Therapy Science. 2012;24(10):949-952. DOI: 10.1589/jpts.24.949

[8] Chapman JP, Chapman LJ, Allen JJ. The measurement of foot preference. Neuropsychologia. 1987;25:579-584. DOI: 10.1016/0028-3932(87)90082-0

[9] Fitts PM. The information capacity of the human motor system in controlling the amplitude of movement. Journal of Experimental Psychology. 1954;47(6):381-391. DOI: $10.1037 /$ h0055392

[10] Kimura J. Electrodiagnosis in Diseases of Nerves and Muscles: Principles and Practice. 2nd ed. Philadelphia: F. A. Davis Company; 2001. pp. 439-443

[11] Mesrati F, Vecchierini MF. F-waves, neurophysiology and clinical value. Neurophysiologic Clinique. 2005;34(5):217-243. DOI: 10.1016/j. neucli.2004.09.005

[12] Sakakibara A, Higashi T, Funase K, Tabira T, Sakai K. Facilitation of the motoneuron excitability induced by remote muscle contraction - Effect of the intensity and the laterality of the muscle contraction (in Japanese). The Journal of Japanese Occupational Therapy Association. 2001;20:45-51

[13] Tabira T, Kusano K, Higashi T, Sakakibara A, Funase K. Facilitation of motor cortex excitability induced by remote muscle contraction (in Japanese). The Journal of Japanese Occupational Therapy Association. 2001;20:465-471

[14] Kiers L, Fernando B, Tomkins D. Facilitatory effect of thinking about movement on magnetic motor-evoked potentials. Electroencephalography 
and Clinical Neurophysiology.

1997;105:262-268. DOI: $10.1016 /$

s0921-884x(97) 00027-1

[15] Hale BS, Raglin JS, Koceja DM.

Effect of mental imagery of a motor task on the Hoffmann reflex. Behavioural Brain Research. 2003;142:81-87. DOI: 10.1016/s0166-4328(02)00397-2

[16] Kasai T, Kawai S, Kawanishi M, Yahagi S. Evidence for facilitation of motor evoked potential (MEPs) induced by motor imagery. Brain Research. 1997;744:147-150,1997. DOI: 10.1016/ s0006-8993(96) 01101-8

[17] Hess CW, Mills KR, Murray NMF. Magnetic stimulation of the human brain: Facilitation of motor responses by voluntary contraction of ipsilateral and contralateral muscles with additional observations on an amputee. Neuroscience Letters. 1986;71(2):235-240. DOI: 10.1016/0304-3940(86)90565-3

[18] Baldissera F, Borroni P, Cavallari P, Cerri G. Excitability changes in human corticospinal projections to forearm muscle during voluntary movement of ipsilateral foot. The Journal of Physiology. 2002;539:903-911. DOI: 10.1113/jphysiol.2001.013282

[19] Henril EH, Naito E, Geyer S, Amunts K, Zilles K, Forssberg H, et al. Simultaneous movements of upper and lower limb are coordinated by motor representations that are shared by both limb: A PET study. The European Journal of Neuroscience. 2000;12:3385-3398. DOI: 10.1046/j.1460-9568.2000.00209.x

[20] Rijntjes M, Dettmers C, Büchel C, Kiebel S, Frackowiak RS, Weiller C. A blueprint for movement: Functional and anatomical representations in the human motor system. The Journal of Neuroscience. 1999;19:8043-8048. DOI: 10.1523/jneurosci.19-18-08043.1999
[21] Kitamura J. Movementrelated cortical potentials and its clinical application (in Japanese). Clinical Electroencephalography. 2004;46:479-486

[22] Winstein CJ, Grafton ST, Pohl PS. Motor task difficulty and brain activity: Investigation of goal-directed reciprocal aiming using positron emission tomography. Journal of Neurophysiology. 1997;77(3):1581-1594

[23] Ugawa Y, Day BL, Rothwell JC, Thompson PD, Merton PA, Marsden CD. Modulation of motor cortical excitability by electrical stimulation over the cerebellum in man. The Journal of Physiology. 1991;441:5772. DOI: 10.1113/jphysiol.1991.sp018738

[24] Takebayashi H, Miyamoto K, Takuma Y, Inoue Y, Miyamoto S, Okabe T, et al. Influence of unilateral muscle force control during bilateral movement on maximum contralateral muscle force (in Japanese). The Journal of Japanese Physical Therapy Association. 2006;33:82-87. DOI: 10.15063/rigaku.KJ00004317394 

Section 3

\section{Relationship between Somatosensory and Motor Research}





\title{
Functional Role of the
} Somatosensory Information to Perceive the Standing Position in the Anteroposterior Direction

\author{
Hitoshi Asai
}

\begin{abstract}
The perceptibility of standing positions in the anteroposterior direction varies according to the standing position. Standing positions with the center of foot pressure (COP) located far from the COP in the quiet standing position show lower stability, and the perceptibility was markedly higher in comparison to positions with the COP near the COP in the quiet standing position. This chapter focuses on the role of somatosensory information in the perception of standing positions in the anteroposterior direction based on our previous study, which concluded that a large change in sensory information generated from the sole of the heel and knee may provide important cues regarding the perception of standing positions with low stability. Large changes in the somatosensory information generated from pressure changes on the sole and from the upward movement of the patella leaning forward or backward while standing may contribute to the position information.
\end{abstract}

Keywords: somatosensory information, perception, standing positions, anteroposterior direction

\section{Introduction}

The control of the standing posture in humans requires various sensory systems, including visual [1], vestibular [2, 3], plantar sole mechanoreceptor [4-8], and proprioceptive [9] systems. The central nervous system (CNS) integrates these different sensory inputs. Upright posture control was performed in various standing positions on the anteroposterior and mediolateral directions. When both arms are rapidly flexed to the front from the quiet standing (QS) posture, the postural muscles (the erector spinae, hamstrings, etc.) are activated 20-30 ms earlier than the focal muscles of the upper extremities (the anterior deltoid) [10]. This earlier activation of the postural muscles in comparison to the focal muscles was defined as the anticipatory postural control to minimize the effect of forthcoming body perturbations due to arm movement [11]. This anticipatory activation of the postural muscles during the QS position was significantly slower than that in the standing position near the most forward-leaning position [10]. Thus, because the preceding time of the earlier activation of the postural muscles varies according to the initial standing position just before arm movement, the initial standing position may be accurately perceived. 
Schiffman described that "Perceptions are associated with the organization and integration of sensory attributes, that is, the awareness of 'things' and 'events' rather than mere attributes or qualities" [12]. Thus, the standing position may be perceived in association with things and events falling and stability. The proprioceptive sensory information concerned with postural control is processed through two routes (cognitive processing and sensorimotor processing) in the CNS [13]. Cognitive processing acts on the perception of the body position [13]. On the other hand, sensorimotor processing regulates the posture via reflex and automatic loops [13]. A previous study reported that the stability of standing posture is higher when the center of the foot pressure in the foot is located 30-60\% from the heel to the length of the foot in the anteroposterior direction [14]. When the center of foot pressure deviates from this range, the stability of standing posture largely decreases [14]. Sensorimotor processing may act strongly in a stable standing position (30-60\% from the heel to the length of the foot), while cognitive processing may operate strongly in an unstable standing position (deviating from this range) in order to maintain safety. It was reported that the perception of the standing position in the anteroposterior direction was accurate in the unstable position [15].

The role of somatosensory information in the perception of standing positions in anteroposterior direction is discussed in this chapter based on our previous studies' findings as follows: (1) the large changes in the plural sensory information from the sole when the center of the foot pressure is located approximately at $70 \%$ of the foot length mutually play an important role in perceiving the standing position; (2) the perception of pressure information at the head of the first metatarsalis reaching a maximum is related strongly to that of pressure information in first toe; (3) in cases in which the patella moves while leaning backward, the patellar movement is accurately perceived; and (4) the large changes in the sensory information that are induced by patellar movement are a cue for the perception of the standing position while leaning backward.

\section{Somatosensory information from the plantar sole when leaning forward while standing}

\subsection{The distribution of foot pressure when the body leans forward and perception of large changes of foot pressure}

The distribution of foot pressure when the body leans slowly forward was reported [4]. When slowly leaning forward from the quiet standing position to the most forward-leaning position, the position at which the pressure at the head of the first metatarsalis showed the maximum value was $71.0 \%$ of the foot length, and the perceived position of this maximum value was $70.5 \%$ of the foot length (Table 1) [4]. There was no significant difference between the two positions. A significant correlation $(r=0.86 ; t=4.99)$ was found between both positions [4]. Thus, this maximum pressure was correctly perceived.

The first toe pressure increased in two forward-leaning standing positions with the subject leaning forward from the quiet standing position. The first pressure increasing position was $60.9 \%$ of the foot length, the second was $69.5 \%$ of the foot length, and the perceived position of the second position was $70.9 \%$ of the foot length [4]. None of the subjects were able to perceive the first large increase in the first toe pressure. A significant correlation $(r=0.92 ; t=7.23)$ was found between the position of the center of pressure in the foot at the second large increase and the perceived position (Table 1) [4]. The second increase of the first toe pressure was correctly perceived as well as that of the first metatarsalis maximum pressure. 
Functional Role of the Somatosensory Information to Perceive the Standing Position... DOI: http://dx.doi.org/10.5772/intechopen.91737

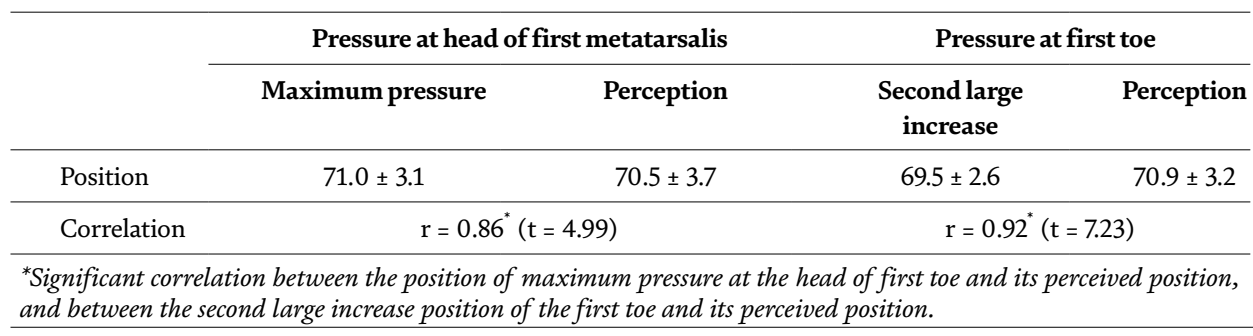

Table 1.

Positions of center of foot pressure (\% of foot length: $\% F L$ ) and correlation: maximum pressure at head of first metatarsalis and second large changes in pressure at first toe (modified from [4]).

Since the standing positions where the first large increase in pressure at the first toe took place when standing were very steady, the probability that the information of this large increase was processed via a sensorimotor processing route is high. The standing position at the second large increase of the first toe was $70 \%$ of the foot length, which was well out of the very steady positional range (30-60\% from the heel to the length of the foot), the probability that the information of this large increase was processed via a cognitive processing route is high.

It is noteworthy that the standing position at the maximum pressure at the first metatarsalis (approximately $70 \%$ of the foot length) is similar to the position of the second large increase in pressure at the first toe, and the correlation between both positions and between both perceived positions was high [4]. At approximately $70 \%$ of the foot length, the foot pressure shifted from the heads of the metatarsalis to the toes on the boundary of this position. The most forward-leaning position is $83 \%$ of the foot length [4]. Approximately $10 \%$ of the foot length is the difference between the standing position at the maximum pressure at the first metatarsalis and the most forward-leaning position. This difference represents an important safety margin when maintaining forward-leaning standing. Thus, the large changes in the plural sensory information from these areas at approximately $70 \%$ of the foot length mutually play an important role in perceiving the standing position through the cognitive processing route [4].

\subsection{Influence of cooling of the sole on the perception of large changes in foot pressure}

To investigate the relationship of the pressure sensation between the first toe and the heads of the metatarsalis, these areas were cooled [4]. A cooling device set at $1^{\circ} \mathrm{C}$ was used to reduce the sensitivity of the first toe or the head of the first metatarsalis. To continue cooling these regions during the measurement period, an iron plate cooled to $1^{\circ} \mathrm{C}$ was placed flush with the floor of the force plate and was changed on every trial (Figure 1) [4].

All subjects $(n=11)$ could perceive the maximum pressure at the head of the first metatarsalis, as described above. When the head of the first metatarsalis was cooled, 8 of 11 subjects could perceive the maximum pressure at the head of the first metatarsalis. When the first toe was cooled, 10 of 11 subjects could perceive the maximum pressure at the head of the first metatarsalis. There were no statistically significant differences among the three conditions (Table 2) [4]. Thus, cooling of the head of the first metatarsalis or the first toe had no effect on the perception of the maximum pressure at the head of the first metatarsalis.

On the other hand, all subjects could perceive the second large increase in first toe pressure, as described above. When the head of the first metatarsalis was 
A

Force platform A

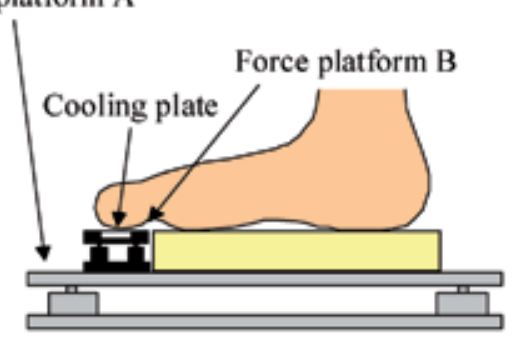

Force platform A

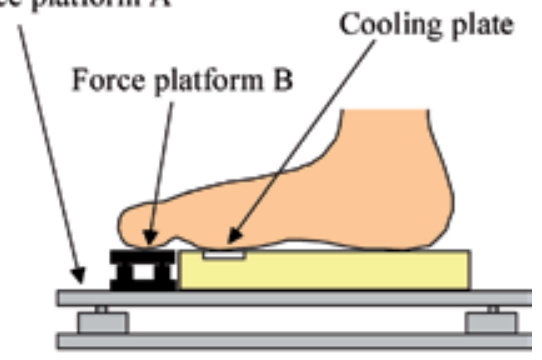

B

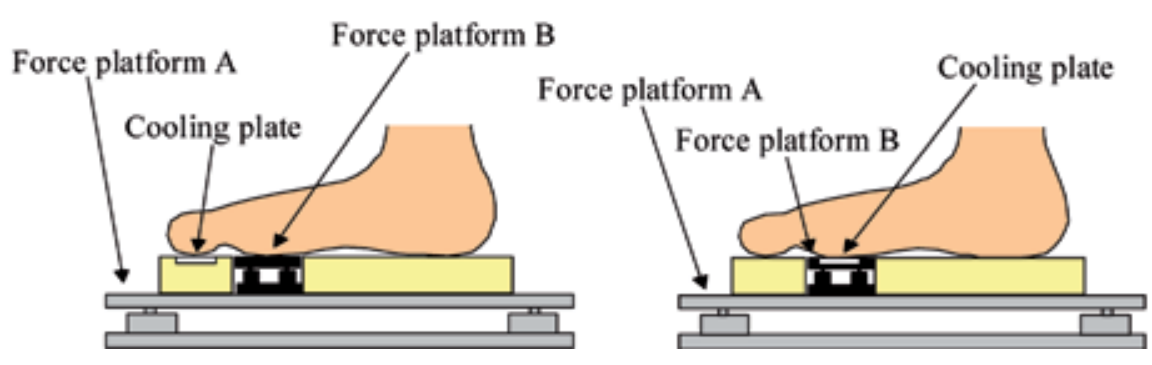

Figure 1.

Diagrams of apparatus. (A) Measuring pressure at the first toe under the cooling condition of the first toe (left panel) and under the cooling condition of the first metatarsalis (right panel). (B) Measuring pressure at the head of the first metatarsalis under the cooling condition of the first toe (left panel) and under the cooling condition of the first metetersalis (right panel) (modified from [4]).

\begin{tabular}{lccc}
\hline Condition & $\begin{array}{c}\text { Pressure at head of first } \\
\text { metatarsalis }\end{array}$ & $\begin{array}{c}\text { Second large increase } \\
\text { at first toe }\end{array}$ \\
\hline Normal (non-cooling) & 11 & 11 \\
\hline Cooling & $\begin{array}{c}\text { Head of first } \\
\text { metatarsalis }\end{array}$ & 8 & $6^{*}$ \\
\cline { 2 - 4 } & First toe & 9 & 9 \\
\hline
\end{tabular}

*Significantly different from the normal condition.

Table 2.

Number of subjects who could perceive the maximum pressure at the head of first metatarsalis, second large increase in first toe pressure under normal and cooling conditions (modified from [4]).

cooled, 6 of 11 subjects could perceive the second large increase in first toe pressure (Table 2) [4]. Thus, cooling the head of the first metatarsalis significantly affected the perception of the second large increase in first toe pressure. When the first toe was cooled, 9 of 11 subjects could perceive the second large increase in first toe pressure (Table 2) [4]. This result suggested that cooling the first toe did not significantly affect the perception of the second large increase at the first toe.

These experiments performed under cooling conditions demonstrated the following: (1) subjects could perceive the maximum pressure at the first metatarsalis when the first metatarsalis was cooled; (2) subjects could perceive the second large increase in first toe pressure when the first toe was cooled, whereas they could not perceive this when the first metatarsalis was cooled. The results suggested that the pressure information at the head of the first metatarsalis reaching a maximum is related strongly to the second large increase of pressure information 
in first toe. Based on this relationship, the second large increase in pressure at the first toe and the maximum pressure at the head of the first metatarsalis are perceived at approximately the same time in the same standing position. In addition, the pressure information at the head of the first metatarsalis may have greater priority in the perception of the second large increase in pressure at the first toe [4].

\section{The upward movement of the patella while leaning backward may contribute to the position information}

\subsection{Perception of upward patellar movement in the backward-leaning standing position}

While gradually leaning backward from the quiet standing position, a large increase of activity in the rectus femoris is typically observed at approximately $30-35 \%$ of the foot length [4]. However, most participants are unable to perceive this large increase [4]. The patella is a sesamoid bone that is connected to the quadriceps tendon, and the knee joint capsule surrounding the patella also adheres to the patella tendon $[16,17]$. Thus, the upward movement of patella may cause deformity of the knee joint capsule and the cutaneous tissues surrounding the knee. Edin reported that sensory information from cutaneous receptors in the anterior thigh close to the knee joint plays an important role in the perception of the knee joint position [18]. The large changes in the somatosensory information produced by the patellar movement should contribute to the perception of a specific backward-leaning standing position. It is considered that participants whose patella moves clearly during backward-leaning standing also clearly perceive large changes in the somatosensory information associated with patellar movement.

Twelve young adult subjects maintained the quiet standing posture for 3 seconds and then slowly moved from their initial standing position to the most backwardleaning standing position over approximately 10 seconds [19]. The experiment was conducted under two conditions. In condition 1, the quadriceps femoris was relaxed (relaxed-start condition). In condition 2, the quadriceps femoris was contracted raising the patella superiorly (contracted-start condition). Four trials were performed under each condition. To analyze the patella range of motion, X-ray exposure was performed under the relaxed-start condition [19].

Under the relaxed-start condition, patellar movement while leaning backward was confirmed in all 4 trials for 8 of the 12 subjects; 3 subjects showed no patellar movement in all 4 trials. Patellar movement was confirmed in 35 of the 48 trials; the mean patellar range of motion in these trials was $9.5 \mathrm{~mm}(\mathrm{SD}=3.0 \mathrm{~mm})$ (Figure 2) [19]. However, two trials in which patellar range of motion was $<3.5 \mathrm{~mm}(9.5 \mathrm{~mm}-$ 2SD) were classified as non-movement trials. Thus, 33 trials were patellar movement trials and 15 were patellar non-movement trials (Table 3) [19]. In the patellar movement trials, 30 trials were perceived and 3 trials were not perceived; thus, $90.9 \%$ of these trials were perceived. On the other hand, $66.7 \%$ of the trials were perceived in the non-movement trials. There was a significant difference between the two rates (Table 3) [19].

Under the contracted-start condition, 37 trials were perceived and 11 trials were not perceived. The rate of the perceived trials under the contracted-start condition was $77.0 \%$. This rate was significantly smaller in comparison to the patellar movement trials performed under the relaxed-start condition (Table 3) [19]. 


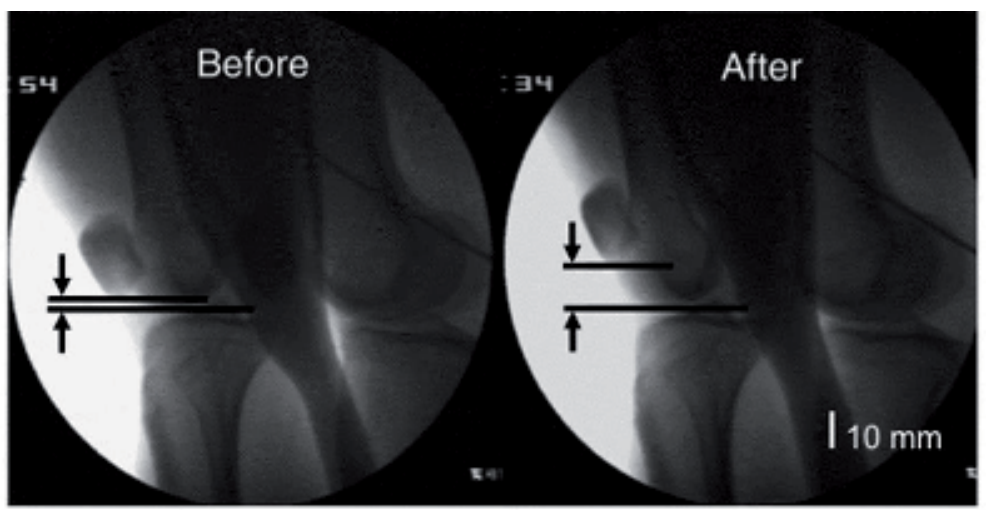

Figure 2.

Typical X-ray photographic image of the patellar movement. Left side: before movement, right side: after movement ([19]).

\begin{tabular}{|c|c|c|c|}
\hline & \multicolumn{2}{|c|}{ The relaxed-start condition } & \multirow{2}{*}{$\begin{array}{l}\text { The contracted-start } \\
\text { condition }\end{array}$} \\
\hline & $\begin{array}{c}\text { Patellar } \\
\text { movement trials }\end{array}$ & $\begin{array}{c}\text { Patellar non- } \\
\text { movement trials }\end{array}$ & \\
\hline Total trials & 33 & 15 & 48 \\
\hline Perceived trials & 30 & 10 & 37 \\
\hline Not perceived trials & 3 & 5 & 11 \\
\hline $\begin{array}{l}\text { Rate of perceived trials } \\
(\%)\end{array}$ & $90.9^{*}$ & 66.7 & 77.0 \\
\hline $\begin{array}{l}\text { "There was a significant diffe } \\
\text { relaxed-start condition. }\end{array}$ & tween patellar mo & rials and patella no & nent trials under the \\
\hline
\end{tabular}

Table 3.

Results of perceptibility of patellar movement in the relaxed-start and the contracted-start conditions (modified from [19]).

Patellar movement in all four trials in the relaxed-start condition was confirmed in eight participants. In contrast, three participants did not show patellar movement in any of the four trials. Thus, there were participants whose patella moved and those whose patella did not move when the subjects were leaning backward. In the patellar non-movement trials, slight flexion of the knees or contraction of the rectus femoris muscle was observed while the participants were in the quiet standing position [19].

The rate of perceived trials under the relaxed-start condition was significantly higher than that of the patellar non-movement trials under the same condition. Conversely, the rate of perceived trials in the patellar non-movement trials under the relaxed-start condition did not differ from that under the contracted-start condition. The contracted-start condition may simulate the patellar non-movement trials under the relaxed-start condition [19].

Mechanoreceptors within the muscles, joint capsules, ligaments, and skin supply information about the joint position and movement contributing to positional perception [18, 20-24]. Thus, in cases in which the patella moves while leaning backward, the patellar movement is accurately perceived through large changes in the sensory information obtained via mechanoreceptors within the muscles, joint capsules, ligaments, and skin around the patella. Consequently, the large changes 
in the sensory information that are induced by patellar movement are a cue for the perception of the standing position while leaning backward.

\subsection{Perception of the position when leaning backward while standing and during patellar movement}

The rectus femoris muscle is significantly activated in the standing position when gradually leaning backward from the quiet standing position and that position is almost constant in each individual [4]. Thus, large changes in somatosensory information based on patellar movement while leaning backward may contribute to the perception of a specific backward-leaning standing position. The perceptibility of the standing position is particularly high near the position of patellar movement, where the large change in sensory information is perceived [25]. Investigating the function of the sensory information based on patellar movement in the perception of backward-leaning standing position is important for understanding the mechanism through which stability is maintained during backward-leaning and through which prevents the individual from falling backward.

Fourteen healthy young adults (six women and eight men) were selected according to three criteria: (1) relaxed quadriceps femoris in the quiet standing position; (2) free movement of patella on palpation in the quiet standing position; and (3) the presence of upward patellar movement while leaning backward. In the experiment, the onset of patellar movement was confirmed using an acceleration waveform generated by an accelerometer taped to the upper edge of the patella [25].

Ten trials were conducted to identify the onset position of patellar movement while leaning backward from the quiet standing position. The onset position of the patellar movement was $35.1 \pm 4.5 \%$ of the foot length. The individual mean value of the standard deviation for the onset position was very small $(2.5 \pm 1.3 \%$ of the foot length) [25].

The perceptibility of three reference positions was evaluated from the reproducibility of these positions, which was determined according to the absolute error between the reference position and the reproduced position. The reference positions set for each participant were as follows: the COP position at the patellar movement onset position (the onset position), $+5 \%$ foot length from the onset position, and $-5 \%$ foot length from the onset position. The mean of $+5 \%$ foot length from the onset position was $40 \%$ of the foot length and that of $-5 \%$ foot length from the onset position was 30\% of the foot length [25]. The mean absolute error at both the $+5 \%$ foot length and the $-5 \%$ foot length from the onset position was defined as the expected value. The ratio of the absolute error in the onset position to the expected value was calculated as the perception error index. A smaller absolute error and smaller perception error index reflect higher perceptibility [25]. The absolute error at $+5 \%$ foot length from the onset position, the onset position, and $-5 \%$ foot length from the onset position were $4.9 \pm 2.00 \%$ of the foot length, $1.9 \pm 0.51 \%$ of the foot length, and $2.7 \pm 0.90 \%$ of the foot length, respectively (Table 4). The reference position was found to have a significant effect on the absolute error $(p<0.001)$. The absolute error values at the onset position and $-5 \%$ foot length from the onset position were significantly smaller in comparison to that at $+5 \%$ foot length from the onset position. The perception error index was $54.1 \pm 18.5 \%$, and this value was significantly smaller than the expected value $(\mathrm{p}<0.001)$ (Figure 3) [25].

Patellar movement was accurately perceived while moving the patella [19]. In this study, the mean standard deviation of the patellar moving onset position was small. Hence, because the onset position is located at a constant position in each 


\begin{tabular}{lccc}
\hline & \multicolumn{3}{c}{ Reference positions } \\
\cline { 2 - 4 } & $\begin{array}{c}\text { The }-5 \% \text { of foot length from } \\
\text { the onset position }\end{array}$ & $\begin{array}{c}\text { The onset } \\
\text { position }\end{array}$ & $\begin{array}{c}\text { The }+5 \% \text { of foot length } \\
\text { from the onset position }\end{array}$ \\
\hline $\begin{array}{l}\text { Absolute error } \\
\text { (\%FL) }\end{array}$ & $2.7 \pm 0.90$ & $1.9 \pm 0.51$ & $4.9 \pm 2.00$ \\
\hline $\begin{array}{l}\text { The perception error } \\
\text { index (\%) }\end{array}$ & $54.1 \pm 18.51^{* *}$ & \\
\hline $\begin{array}{l}\text { "Statistically smaller than the }+5 \% F L \text { from the onset position. } \\
{ }^{*} \text { Statistically smaller than the expected value (100\%). }\end{array}$ &
\end{tabular}

Table 4.

Absolute error in three reference positions and the perception error index in the onset position (modified from $[25])$.

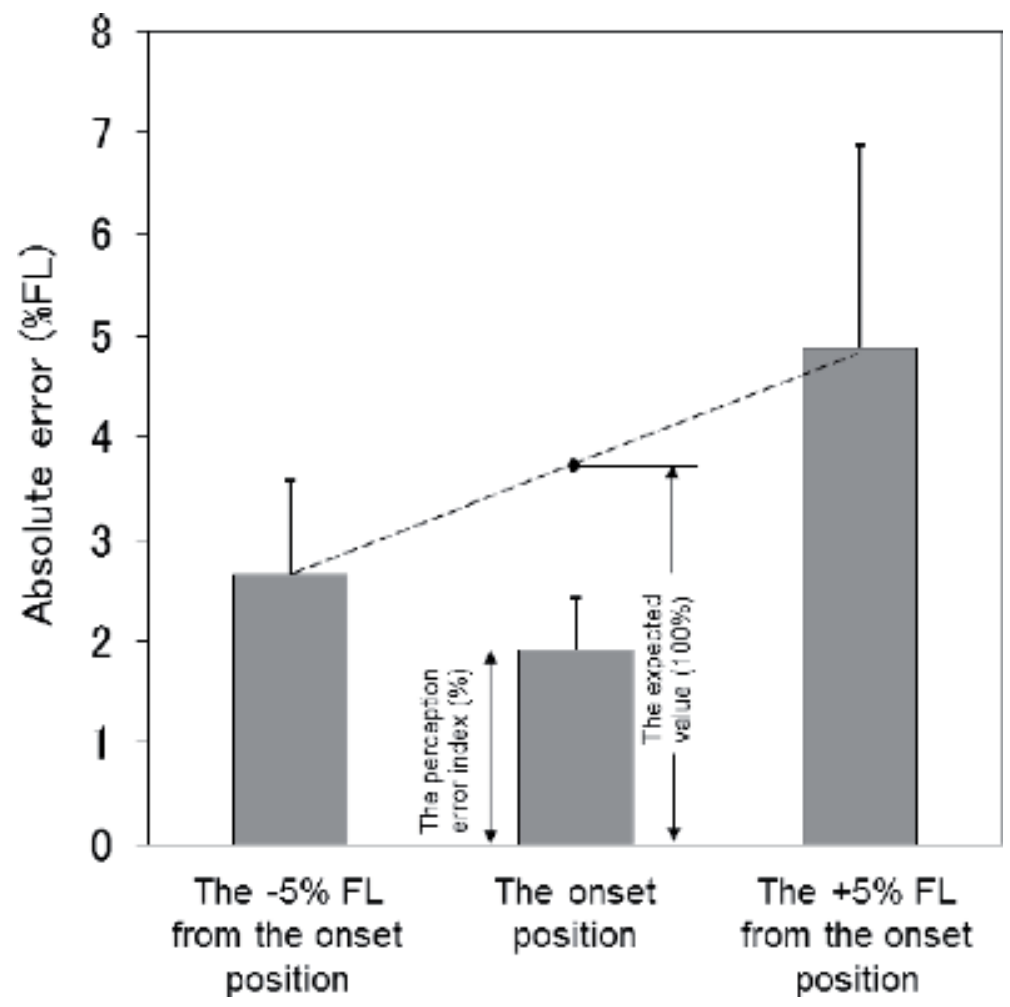

Figure 3.

Absolute error of the three reference positions, and the calculation method for perception error index ([25]).

participant, the large change of sensory information should play an important role in the individual's postural stability at the onset position. It is considered that the standing position is accurately perceived by building up the sensory reference frame based on the large change of sensory information caused by the patellar movement [25]. The onset position (approximately $35 \%$ of the foot length) is located near the posterior end of the stability range for the standing position. As a result, the absolute error in the onset position was significantly smaller than that at $+5 \%$ of the foot length from the onset position. In addition, the absolute error in the onset position was same as that in position about $25 \%$ of the foot length. The heel pressure distribution was reported to change substantially approximately near $25 \%$ of the foot length and the perceptibility of this change was highly accurate [26]. Since 
stability of the standing posture reduces posteriorly to the end of this range [27], the large increase in sensory information associated with patellar movement when leaning backward while standing may represent warning information [25].

\subsection{Relevance and application to physical therapy}

In this chapter, the role of the somatosensory information in the perception of the standing positions in the anteroposterior direction is discussed based on the findings of our previous study.

During forward-leaning standing, the large changes of somatosensory information from the metatarsalis and the toes may have an important role in the perception of the standing position. The relationship between the pressure information of the first toe and the first metatarsalis is particularly strong to perceive the standing position at approximately $70 \%$ of the foot length. During backward-leaning standing, the large changes in somatosensory information associated with the onset of patellar movement may have an important role in the perception of the standing position at the posterior end of the stability range for the standing position.

The somatosensory information for the perception of the body position for motor control is important for physical therapy. Thus, physical therapists should consider which sensory information is weighted and which sensory information is re-weighted by CNS to perceive the body position on the reference frame for balance control in patients.

\section{Conclusion}

This chapter showed and discussed the important role of the somatosensory information from the sole and around the patella to perceive the standing position in anteroposterior direction. During forward-leaning standing, the large changes in the pressure information at the head of the first metatarsalis and the first toe at approximately $70 \%$ of the foot length mutually play an important role in perceiving the standing position. The perception of pressure information at the head of the first metatarsalis reaching a maximum is strongly related to that of pressure information in first toe. In cases in which the patella moves while gradually leaning backward from the quiet standing position, the upward movement of patella may cause deformity of the knee joint capsule and the cutaneous tissues surrounding the knee. The mean standard deviation of the patellar moving onset position was small. Hence, because the onset position is located at a constant position in each participant, the large changes in the sensory information induced by patellar movement are a cue for the perception of the standing position while leaning backward.

\section{Conflicts of interest}

The author has no conflicts of interest directly relevant to the content of this chapter. 


\section{Author details}

Hitoshi Asai

Department of Physical Therapy, Graduate Course of Rehabilitation Science, School of Health Sciences, College of Medical, Pharmaceutical, and Health Sciences, Kanazawa University, Kanazawa, Japan

*Address all correspondence to: asai@mhs.mp.kanazawa-u.ac.jp

\section{IntechOpen}

(C) 2020 The Author(s). Licensee IntechOpen. This chapter is distributed under the terms of the Creative Commons Attribution License (http://creativecommons.org/licenses/ by/3.0), which permits unrestricted use, distribution, and reproduction in any medium, provided the original work is properly cited. (cc) BY 
Functional Role of the Somatosensory Information to Perceive the Standing Position...

DOI: http://dx.doi.org/10.5772/intechopen.91737

\section{References}

[1] Paulus WM, Straube A, Brandt T. Visual stabilization of posture. Physiological stimulus characteristics and clinical aspects. Journal of Neurology. 1984;107(Pt 4):1143-1163

[2] Cathers I, Day BL, Fitzpatrick RC. Otolith and canal reflexes in human standing. The Journal of Physiology. 2005;563(Pt 1):229-234

[3] Horak FB, Shupert CL, Dietz V, Horstmann G. Vestibular and somatosensory contributions to responses to head and body displacements in stance. Experimental Brain Research. 1994;100:93-106

[4] Asai H, Fujiwara K. Perceptibility of large and sequential changes in somatosensory information during leaning forward and backward when standing. Perceptual and Motor Skills. 2003;96:549-577

[5] Kavounoudias A, Roll R, Roll JP. Specific whole-body shifts induced by frequency-modulated vibrations of human plantar soles. Neuroscience Letters. 1999;266:181-184

[6] Kavounoudias A, Roll R, Roll JP. Foot sole and ankle muscle inputs contribute jointly to human erect posture regulation. The Journal of Physiology. 2001;532:869-878

[7] Billot M, Handrigan GA, Simoneau M, Corbeil P, Teasdale N. Short term alteration of balance control after a reduction of plantar mechanoreceptor sensation through cooling. Neuroscience Letters. 2013;535:40-44

[8] Billot M, Handrigan GA, Simoneau M, Teasdale N. Reduced plantar sole sensitivity induces balance control modifications to compensate ankle tendon vibration and vision deprivation. Journal of
Electromyography and Kinesiology. 2015;25:155-160

[9] van Deursen RW, Simoneau GG. Foot and ankle sensory neuropathy, proprioception, and postural stability. The Journal of Orthopaedic and Sports Physical Therapy. 1999;29:718-726

[10] Fujiwara K, Toyama H, Kunita K. Anticipatory activation of postural muscles associated with bilateral arm flexion in subjects with different quiet standing positions. Gait \& Posture. 2003;17:254-263

[11] Santos MJ, Kanekar N, Aruin AS. The role of anticipatory postural adjustments in compensatory control of posture: 1. Electromyographic analysis. Journal of Electromyography \& Kinesiology. 2010;20:388-397. DOI: 10.1016/j.jelekin.2009.06.006

[12] Schiffman HR. Sensation and Perception. 3rd ed. New York: John Wiley \& Sons; 1990. pp. 1-9

[13] Roll R, Gilhodes JC, Roll JP, Povov K. Proprioceptive information processing in weightlessness. Experimental Brain Research. 1998;122:393-402

[14] Fujiwara K, Ikegami H, Okada M. The relationship between the postural stability and the relative muscle load of lower limbs in upright stance. Bulletin of Health and Sports Sciences Univesity of Tsukuba. 1985;8:165-171. [in Japanese with English abstract]

[15] Fujiwara K, Asai H, Toyama H, Kunita K. Perceptibility of body position in anteroposterior direction while standing with eyes closed. Perceptual and Motor Skills. 1999;88:581-589

[16] Kapandji IA. Physiology of the Joints. Lower Limb. Volume 2. 5th ed. New York: Elsevier Health Sciences; 1987 
[17] Standring S. Gray's Anatomy. The Anatomical Basis of Clinical Practice, Expert Consult. 40th ed. Philadelphia: Churchill Livingstone; 2008

[18] Edin B. Cutaneous afferents provide information about knee joint movements in humans. The Journal of Physiology. 2001;531:289-297

[19] Asai H, Odashiro Y, Inaoka PT. Patellar movement perception related to a backward-leaning standing position. Journal of Physical Therapy Science. 2017;29:1372-1376. DOI: 10.1589/ jpts. 29.1372

[20] Barrack RL, Skinner HB, Brunet ME, Haddad RJ Jr. Functional performance of the knee after intraarticular anesthesia. The American Journal of Sports Medicine. 1983;11:258-261

[21] Clark FJ, Horch KW, Bach SM, Larson GF. Contributions of cutaneous and joint receptors to static kneeposition sense in man. Journal of Neurophysiology. 1979;42:877-888

[22] Collins DF, Refshauge KM, Todd G, Gandevia SC. Cutaneous receptors contribute to kinesthesia at the index finger, elbow, and knee. Journal of Neurophysiology. 2005;94:1699-1706

[23] Isaac SM, Barker KL, Danial IN, Beard DJ, Dodd CA, Murray DW. Does arthroplasty type influence knee joint proprioception? A longitudinal prospective study comparing total and unicompartmental arthroplasty. The Knee. 2007;14:212-217

[24] McCloskey DI, Cross MJ, Honner R, Potter EK. Sensory effects of pulling or vibrating exposed tendons in man. Brain. 1983;106:21-37

[25] Asai H, Hirayama K, Azuma Y, Inaoka PT. Perception of leaning backward while standing and patellar movement. Journal of Physical Therapy
Science. 2017;29:1670-1674. DOI: 10.1589/jpts.29.1670

[26] Fujiwara K, Asai H, Koshida K, Maeda K, Toyama H. Perception of large change in distribution of heel pressure during backward leaning. Perceptual and Motor Skills. 2005;100:432-442

[27] Fujiwara K, Ikegami H, Okada M. The position of the center of foot pressure in an upright stance and its determining factors. Japanese Journal of Human Posture. 1984;4:9-16. [in Japanese with English abstract] 



\section{Edited by Toshiaki Suzuki}

Motor functions such as muscle strength and joint range of motion as well as

somatosensory functions that enable a person to interpret bodily sensation are necessary and important for physical activity and exercise. Somatosensory and Motor Research is divided into three sections: "Somatosensory Research," "Motor Research," and "Relationship between Somatosensory and Motor Research." Written by researchers and experts in the field, chapters in this volume address basic anatomy and physiology, treatment of sensory disorders, motor function, and somatic sensations. This book contributes to the understanding of both motor nerve and sensory nerve function and their involvement in the acquisition of movement.

\section{IntechOpen}

